\title{
Electrodynamics of Magnetars: \\ Implications for the Persistent X-ray Emission and Spindown of the Soft Gamma Repeaters and Anomalous X-ray Pulsars
}

\author{
C. Thompson ${ }^{1}$, M. Lyutikov ${ }^{2,3,4}$, and S.R. Kulkarni ${ }^{5}$ \\ Submitted to the Astrophysical Journal
}

\begin{abstract}
We consider the structure of neutron star magnetospheres threaded by large-scale electrical currents, and the effect of resonant Compton scattering by the charge carriers (both electrons and ions) on the emergent X-ray spectra and pulse profiles. In the magnetar model for the Soft Gamma Repeaters and Anomalous X-ray Pulsars, these currents are maintained by magnetic stresses acting deep inside the star, which generate both sudden disruptions (SGR outbursts) and more gradual plastic deformations of the rigid crust. We construct self-similar force-free equilibria of the current-carrying magnetosphere with a power law dependence of magnetic field on radius, $\mathbf{B} \propto r^{-(2+p)}$, and show that a large-scale twist of field lines softens the radial dependence of the magnetic field to $p<1$. The spindown torque acting on the star is thereby increased in comparison with an orthogonal vacuum dipole. We comment on the
\end{abstract}

\footnotetext{
${ }^{1}$ Canadian Institute for Theoretical Astrophysics, 60 St. George St., Toronto, ON M5S 3H8

${ }^{2}$ Department of Physics, McGill University, Montréal, QC

${ }^{3}$ Massachusetts Institute of Technology, 77 Massachusetts Avenue, Cambridge, MA 02139

${ }^{4}$ CITA National Fellow

${ }^{5}$ California Institute of Technology, 105-24, Pasadena, CA 91125
} 
strength of the surface magnetic field in the SGR and AXP sources, as inferred from their measured spindown rates, and the implications of this model for the narrow measured distribution of spin periods.

A magnetosphere with a strong twist $\left(B_{\phi} / B_{\theta}=O(1)\right.$ at the equator) has an optical depth $\sim 1$ to resonant cyclotron scattering, independent of frequency (radius), surface magnetic field strength, or charge/mass ratio of the scattering charge. When electrons and ions supply the current, the stellar surface is also heated by the impacting charges at a rate comparable to the observed X-ray output of the SGR and AXP sources, if $B_{\text {dipole }} \sim 10^{14} \mathrm{G}$. Redistribution of the emerging X-ray flux at the cyclotron resonance will strongly modify the emerging pulse profile and, through the Doppler effect, generate a non-thermal tail to the X-ray spectrum. We relate the sudden change in the pulse profile of SGR 1900+14 following the 27 August 1998 giant flare, to an enhanced optical depth at the electron cyclotron resonance resulting from a sudden twist imparted to the external magnetic field during the flare. The self-similar structure of the magnetosphere should generate frequency-independent profiles; more complicated pulse profiles may reflect the presence of higher multipoles, ion cyclotron scattering, or possibly non-resonant Compton scattering of O-mode photons by pair-loaded currents.

Subject Headings: gamma rays: bursts - stars: neutron - X-rays: stars

\section{Introduction}

Our view of neutron stars has been strongly influenced by radio pulsars. The demographics and physical properties of isolated neutron stars have largely been derived from extensive pulsar searches, and associations of pulsars with supernovae. Over the last decade, thanks to new high energy missions, there has been a growing recognition of diversity in the neutron star population. Considerable attention has been focused on the radio-quiet neutron stars (RQNS), which often have a bright presence in the X-ray sky (Frail 1998). Although the known RQNS are certainly heterogeneous, two groups of objects seem to show sufficient regularity to classify them: the Anomalous X-ray pulsars (AXPs) and the Soft Gamma-ray Repeaters (SGRs). Both appear to be young objects given their location within supernova remnants and star-forming regions.

The AXPs have spin periods in the range $P=6-12 \mathrm{~s}$, characteristic ages $P / \dot{P}=3 \times 10^{3}-4 \times 10^{5} \mathrm{yr}$, and X-ray luminosities $L_{X}=5 \times 10^{34}-10^{36} \mathrm{erg} \mathrm{s}^{-1}$ (for recent reviews see Mereghetti 2000, Thompson 2001). Three out of the six AXPs lie close 
to the centers of SNRs, confirming their youth. In none of these objects is there evidence of a binary stellar companion, or the usual indicators of accretion through a disk. Their spin-down luminosity is always less than a few percent of the observed X-ray luminosity. The unfamiliar source of energy makes these objects anomalous.

SGRs are identified by their hard X-ray flares. Once localized, their quiescent X-ray emission (also in the range $L_{X} \sim 5 \times 10^{34}-10^{36} \mathrm{erg} / \mathrm{s}$ ) allows further studies of these equally enigmatic objects. SGRs have spin periods in the range 5-8 s but somewhat smaller characteristic ages than the AXPs, less than a few thousand years. Only one or two of the four SGRs are concident with a SNR. However, all the SGRs are found in star-forming regions, attesting to their youth. It is important to note that only $50 \%$ of pulsars with characteristic ages less than $\sim 5 \times 10^{4}$ years have associated SNRs. Thus, for both SGRs and AXPs, the frequency of associations with SNRs appears no different statistically from that of young pulsars (Gaensler et al. 2001). As with AXPs, there are no observational reasons to believe that these objects have companions; nor evidence for accretion. The reader is referred to Hurley (2000) and Thompson (2000) for recent reviews of the SGRs.

The giant flare of 5 March 1979 (Mazets et al. 1979) now associated with SGR 0525-66 provided the first observational evidence that the SGRs are highly magnetized neutron stars or "magnetars". These objects where hypothesized to form through dynamo action in supernova collapse (Duncan \& Thompson 1992). Superstrong magnetic fields, $B>10^{15} \mathrm{G}$, are able to supply enough energy to power the bursts, thereby matching the extreme peak luminosities of more a million times Eddington; and to confine a significant proportion of the cooling plasma over the long (> $200 \mathrm{~s}$ ) duration of the flare (Duncan \& Thompson 1992; Pacyzński 1992; Thompson \& Duncan 1995). The presence of 8-s pulsations in the March 5 burst provided a consistent and independent support for the magnetar hypothesis.

The discovery of long period pulsations in the quiescent X-ray emission of SGRs by Kouveliotou et al. (1998) has opened a rich field of timing studies of SGRs (Woods et al. 1999c, 2001b; Kaspi et al. 1999, 2001; Gavriil \& Kaspi 2001), from which we are now able to obtain critical information about both the electrodynamics and the superfluid behavior of these interesting objects, in much the same way as has been the case with timing studies of radio pulsars.

The same magnetar model which has successfully described the bright outbursts of the SGRs is also able to account for their long spin periods and their quiescent emission (through magnetic field decay). Indeed, the SGRs in their quiescent states overlap with the AXPs in a three-dimensional parameter space $\left(P, \dot{P}\right.$ and $\left.L_{X}\right)$. This striking similarity motivated the suggestion that they share a common energy source: the decay of a very strong magnetic field (Thompson \& Duncan 1996, hereafter TD96). The bursting activity 
of the SGRs is highly intermittent, so that some of the AXPs may be dormant SGRs.

The circumstantial connection between the SGRs and AXPs has become stronger with two objects SGR 0526-66 and AXP 1E 1048.1-5937 constituting as possible "missing links'. After the 5 March 1979 giant flare, SGR 0526-66 continued to emit a dozen shorter bursts, but has not been an active burster since 1983. Its persistent spectrum is consistent with a pure power law with photon index $15=3.5$ (Kulkarni et al. 2001). This relatively soft power-law index is characteristic of the AXPs, but is much softer than the $\Gamma \simeq 2.2$ measured in the persistent emission of the other SGRs. By contrast, 1E 1048.1-5937 has never been observed to burst, but its power-law index $\Gamma=2.5$ is harder than that of SGR 0526-66. Equally significantly, this object appears to have the largest timing noise of AXPs and at levels similar to those of SGRs (Kaspi et al. 2001). Specifically, between 1994 and 1996, the spindown torque of 1E 1048.1-5937 appears to have increased by a factor $\sim 2$ over its long term value (Paul et al. 2000). This behavior is similar to the accelerating spindown observed in SGR 1900+14 several months following the August 27 giant flare (Woods et al. 2001b). Thus, the latest timing results are unveiling a remarkable similarly in the detailed spin behavior of the SGRs and AXPs.

Within the combined population of SGR and AXP sources, there is overall a positive correlation between spindown rate and the hardness of the spectral index $\Gamma$ in the persistent emission (Marsden \& White 2001). There is, similarly, a positive correlation between hardness and pulsed fraction within the AXP population (e.g. Table 2 in Kaspi et al. 2001).

This smooth continuity of the X-ray and timing properties, when combined with the other observational similarities summarized above, provides a strong phenomenological link between SGRs and AXPs. If the SGRs are magnetars, then so are the AXPs.

The discovery of optical counterparts of AXPs (Hulleman, van Kerkwijk \& Kulkarni 2000; Hulleman et al. 2001) offers new clues about AXPs. The existing optical data on SGRs are consistent with SGR counterparts being similarly faint (Kaplan et al. 2001a). These authors find that the dimness of the optical counterpart is incompatible with standard accretion disk models and thus by elimination provide evidence for the magnetar model.

There do, however, exist significant differences between AXPs and SGRs. Beyond the obvious difference - the spectacular X-ray outbursts of the SGRs - there is also a puzzling discrepency between different methods of estimating their relative ages. The location of three AXPs close to the centers of their SNRs would suggest that - given similar proper

\footnotetext{
${ }^{6}$ Here $d N / d E \propto E^{-\Gamma}$.
} 
motions - the SGRs must descend from the AXPs. However, the characteristic ages of SGRs are typically smaller than those of AXPs. Combining these two observations, one infers that the measured spindown of an SGR must be transiently accelerated compared with its long-term average. Precisely this effect is now being observed (over relatively brief 1-2 year intervals) in the timing solutions (Paul et al. 2000; Woods et al. 2001b). The positive correlation between spindown rate and cumulative bursting activity further suggests that activity as an SGR source is also intermittent. One of the basic goals of this paper is to connect this apparent transient behavior to the magnetar model.

This general picture does not require a temporal link between the SGRs and AXPs, but if one exists then the SGRs are probably old AXPs which hibernate most of the time and display SGR-like activity over a small fraction $(\sim 10-25 \%)$ of their lifetime. Alternatively, some AXPs may never emit bright X-ray outbursts, and may be distinguished from the SGRs in some hidden attribute, e.g., the multipolarity of the magnetic field.

How do the AXPs and SGRs fit into the grander picture of neutron star behavior? Recent radio surveys have discovered pulsars with polar magnetic fields approaching $10^{14} \mathrm{G}$ (Camilo 2000), continuous with the lower range of fields deduced from AXP spindown. However, these objects appear to be no different from other radio pulsars and in particular are not X-ray bright (Pivovaroff et al. 2000). This bifurcation in X-ray behavior suggests that the magnetar phenomenon (e.g. rapid magnetic field decay) does not turn on until $B$ exceeds $10^{14} \mathrm{G}$. In particular, AXPs and radio pulsars may be distinguished by the strength of the internal magnetic field.

In this paper, we present a physical model which synthesizes the persistent emission and torque behavior of the SGRs and AXPs, relates that behavior to the mechanism driving the SGR flares, and provides a strong suggestion that these objects were born with very rapid rotation. The starting hypothesis is that the magnetic field is globally twisted inside the star - up to a strength about 10 times the external dipole - and is strong enough to twist up the external field (at intervals). In the magnetar model, these currents provide the most promising mechanism for generating the non-thermal persistent emission, through magnetospheric Comptonization and surface heating (TD96). Direct evidence for this effect comes from the observed change in the pulse profile of SGR 1900+14 following the 27 August 1998 giant flare (Thompson et al. 2000). We explain how these persistent currents flowing outside the star will heat its surface, and modify the emerging X-ray spectrum and pulse profile. The external twist will also increase the electrical current flowing across the speed of light cylinder, and therefore the spindown torque acting on the star. A positive correlation between spectral hardness and spindown rate is a natural consequence of this model. 
The presence of an ultrastrong magnetic field, $B \sim 4 \times 10^{15} \mathrm{G}$, in the deep interior of the SGR and AXP sources has a few further interesting consequences.

First, such a strong field can comfortably account for the total energy released by these sources in various channels. Not only do magnetars lose energy through electromagnetic emissions (X-ray, UV and optical), but also through relativistic particles and neutrino radiation from the deep crust and core. There is evidence for particle losses during the 27 August 1998 giant burst of SGR 1900+14 (Frail, Kulkarni \& Bloom 1999). While current observations do not show any plerions around other SGRs (or AXPs), the upper limits on the persistent particle luminosity obtained from these observations are not constraining. If the observed X-ray output of the AXPs is dominated by transport of the magnetic field through the neutron star core, then neutrino losses will exceed the surface flux by a factor $\sim 30$ (TD96; Heyl \& Kulkarni 1998). Estimating the bolometric losses is also complicated in some sources (primarily the AXPs) by a steep power-law component in the X-ray spectrum, which is not observed below $\sim 0.5 \mathrm{keV}$ due to absorption. Assuming a typical observed luminosity of $3 \times 10^{35} \mathrm{erg} \mathrm{s}^{-1}$ and a mean age of $10^{4} \mathrm{yr}$ we obtain $E \sim 10^{47} \mathrm{erg}$, comparable to the inferred output of the bursting SGR sources. This should be compared to the total magnetic energy of $10^{47} B_{15}^{2}$ erg where $B_{15}$ is the strength of the field in units of $10^{15} \mathrm{G}$ and it is assumed that this field permeates the entire volume of the neutron star. The very high electrical conductivities of neutron star interiors will prevent currents from being entirely dissipated, and so internal fields somewhat stronger than $\sim 10^{15} \mathrm{G}$ are needed to supply the entire bolometric output of an AXP or SGR source. This simple estimate also underlines the importance of obtaining more precise measurements of the low-energy spectral cutoff in sources with soft spectra.

Second, the presence of ultrastrong internal magnetic fields leads to a simple physical mechanism for the termination of the bright X-ray emissions of the SGR and AXP sources. It is clear observationally that the AXP/SGR phenomenon is restricted to young objects, with total ages less than $10^{4}-10^{5} \mathrm{yr}$. What causes this decline in magnetar activity? One explanation involves the strong temperature-dependence of the rate of magnetic field transport through the neutron star core (e.g. Goldreich \& Reisenegger 1992). Transport is dramatically accelerated by internal heating, and so the transport of a deeply anchored field will cut off sharply when cooling of the star is dominated by surface X-ray emission (TD96).

What do old magnetars look like? It is in this context that the discovery of long period isolated nearby X-ray pulsars such as RX J0720.4-3125 (Haberl et al. 1997) becomes interesting. There are three such objects (see Treves et al. 2000 for a review), a high fraction $(30 \%)$ of nearby neutron stars. Several authors have argued these objects to be old magnetars (e.g. Heyl \& Hernquist 1998, Kulkarni \& van Kerkwijk 1998). If indeed 
this is the case, then the birthrate of magnetars is a significant fraction of the pulsar birth rate. Furthermore, the similarity of the periods of these objects with those of AXPs+SGRs indicates that period evolution may slow down after the active magnetar phase.

Third, as we have already mentioned, the presence or absence of a strong toroidal field offers an explanation for the bifurcation in behavior between radio pulsars and the X-ray bright SGRs and AXPs. More generally, the maximum total (as opposed to dipolar) field which will manifest pulsar-like activity (and the minimum field which is needed to power an AXP or SGR) needs a more detailed theoretical explanation. These minima and maxima need not coincide. Is there a gap between the B-field strengths of the observed high-field pulsars, and the magnetar population? The theoretical model advanced in this paper will be useful in making this question more precise.

\subsection{Plan of the Paper}

In this paper, we consider the properties of neutron stars whose external magnetic fields support global electrical currents, flowing across a large fraction of the stellar surface. We construct axisymmetric, self-similar solutions to the force-free equation, which describe the structure of a magnetosphere with a global twist between the two hemispheres. We begin in Section 2 with a general discussion of how the decay of an electrical current flowing inside stellar material of a very high electrical conductivity may be accelerated as hydromagnetic stresses divert part of the current to the exterior, where it is damped much more rapidly. Section 3 describes the construction and basic properties of our self-similar model, which forms a one-parameter sequence labeled by the net angle through which the magnetic field lines are twisted (or, equivalently, by the radial power-law index of the magnetic field). The modification of the corotation charge density by static currents is calculated, and the matching of our twisted solution onto open magnetic field lines is discussed qualitatively.

Resonant cyclotron scattering of photons by the charge carriers is explored in Section 4 , where the following remarkable property is demonstrated: the optical depth to resonant scattering is proportional to the twist, but does not depend explicity on the radius, or the mass and electrical charge of the current-carrying particles. This resonant optical depth is largest at the magnetic equator, but decreases to zero near the magnetic axis (where the current flowing along more extended field lines is relatively weak).

Section 5 gives an introduction to how the X-ray spectrum and pulse profile of a magnetar will be modified by multiple resonant scattering in the magnetosphere, as well as the implication for the mechanism of the giant flares in the SGR sources. In Section 6, 
we calculate how the surface field inferred from spindown measurements is modified, and then consider the implications of our model for the narrow distribution of pulse periods measured in the SGR and AXP sources. Section 7 closes the paper with a summary of our findings, and their more general implications for the nature of the SGR and AXP sources.

\section{Twisted Neutron Star Magnetospheres}

The non-thermal persistent emission of the SGRs has been ascribed to a static twist imparted to the external magnetic field by sub-surface motions during X-ray flares, with the effect of diverting an electrical current from the interior of the star to its exterior (Thompson et al. 2000). Magnetic fields exceeding $\sim 10^{14} \mathrm{G}$ are strong enough to fracture the deep crust of a neutron star and, if stronger than $\sim 10^{15} \mathrm{G}$, will undergo rapid transport through the dense stellar interior over the short $\sim 10^{4}-10^{5} \mathrm{yr}$ active lifetime of the SGR/AXP sources (TD96; Heyl \& Kulkarni 1998).

The magnetic fields of neutron stars are most likely generated by a hydromagnetic dynamo as the star is born. At this time, magnetic fields are easily transported across the boundary of the neutron-rich core, where they can reconnect from the field anchored in the star (Thompson \& Murray 2001). In this manner, net magnetic helicity builds up in the anchored field as the disconnected field carries away helicity. Note also that the net twist which is maintained along extended field lines (reaching out beyond several stellar radii) is controlled by the current flowing through only a fraction of the surface.

Consider a twisted magnetic field which is anchored in a highly conducting crust of a neutron star (Fig. 11). Suppose that initially the twist vanishes outside the star, where the conductivity is much lower. Then the current closes through a thin surface layer, which feels a Lorentz force $\frac{1}{c} \mathbf{J} \times \mathbf{B}$. In the absence of any tensile strength, this force causes the surface layer and the external field to twist up. The net effect is to distribute the twist more uniformly along the flux tube (Fig. 11), and to hasten the decay of the current by forcing it into a medium of lower conductivity - the magnetosphere. The twist will then relax in a time comparable to the decay time $t_{\text {decay }}^{\text {ex }}$ outside the star. In the case of a realistic neutron star, this process will be impeded by the compositional stratification of the stellar core: the untwisting of the magnetic field is suppressed if it requires the motion of fluid elements across equipotential surfaces (Thompson \& Duncan 2001).

In the SGRs and AXPs, such an external current can be supported by charges stripped from the surface: the effective temperature is high enough to allow thermal emission of electrons and light ions (hydrogen and helium), even in the presence of a $\sim 10^{15} \mathrm{G}$ magnetic 
field (Thompson et al. 2000). Any large-scale deformation of a neutron star is further constrained by the rigidity of its crust. A weak magnetic field is pinned by the crust, and the external current decays without being replenished. The behavior of magnetic fields stronger than $\sim 10^{14}-10^{15} \mathrm{G}$ depends on the intermittency with which the crust responds to the applied magnetic stresses. The compositional stratification of a neutron star (Reisenegger \& Goldreich 1992) strictly limits the degree to which an internal magnetic field can unwind promptly. The lowest energy deformations of the rigid crust are rotational, and large-scale fractures can be triggered repeatedly as transport processes (such as ambipolar diffusion and Hall drift) allow the shear components of the magnetic stress to build up with time. An empirical estimate of this growth time is given by the mean duration $\Delta t_{\text {flare }} \sim 10^{2} \mathrm{yr}$ between giant flares in any one SGR source (e.g. Mazets et al. 1999). The external current will be maintained more or less continuously if $t_{\text {decay }}^{\text {ex }} z t_{\text {growth }} \sim \Delta t_{\text {flare }}$; otherwise, the external current will decay in between spasmodic events. More gradual, plastic deformations of the star are also possible and, in the magnetar model, are required to explain measured variations in the X-ray output of some AXPs (e.g. Iwasawa, Koyama, \& Halpern 1992) which are not associated with bright X-ray bursts. In this case, a non-potential magnetic field will be maintained outside the star only if $t_{\text {decay }}^{\text {ex }} z t_{\text {growth }}$.

\section{Force-Free Equilibria}

The exterior of an isolated neutron star is traditionally modeled as a potential magnetic field, excluding a narrow bundle of field lines which extend out to the speed of light cylinder (Pacini 1967; Goldreich \& Julian 1969; Ruderman \& Sutherland 1975). We generalize this classical dipole model to include an electrical current flowing continuously across the entire surface of the star. We assume initially that the star does not rotate, so that the closed magnetic field lines fill an infinite volume outside the star. The effects of slow rotation are discussed in Sections 3.1 and 3.2 .

The energy density of the charge carriers needed to supply the current is, in this context, tiny compared with $B^{2} / 8 \pi$, and the bare space charge $|\rho| \ll J / c$ on the closed field lines. This leads us to solve the force-free equation, $\mathbf{J} \times \mathbf{B}=0$, to determine the structure of the magnetic field around a highly conducting spherical mass of radius $R_{\mathrm{NS}}$. The solution of this equation can be written formally as

$$
\nabla \times \mathbf{B}=\alpha(\mathcal{P}) \mathbf{B}
$$

When the magnetosphere is axisymmetric (as we will assume), the magnetic field lines form a one-dimensional sequence labeled by the flux parameter $\mathcal{P}=\mathcal{P}(R, \theta)$. The poloidal 
magnetic field can be written as

$$
\mathbf{B}_{P}=\frac{\nabla \mathcal{P} \times \hat{\phi}}{R \sin \theta} .
$$

(In components, $B_{R}=-R^{-2} \partial \mathcal{P} / \partial(\cos \theta)$ and $B_{\theta}=-(R \sin \theta)^{-1} \partial \mathcal{P} / \partial R$.) The constancy of the current along an infinitesimal bundle of magnetic flux guarantees that the coefficient $\alpha$ is a function only of $\mathcal{P}$. As a major simplification we search for self-similar configurations

$$
\mathcal{P}=\mathcal{P}_{0} r^{-p} F(\cos \theta),
$$

adapting the trick of Lynden-Bell and Boily (1994) who studied force-free equilibria bounded by an infinite, conducting plane. Here, $r=R / R_{\mathrm{NS}}, \theta$ is the magnetic co-latitude, and $\mathcal{P}_{0}=\frac{1}{2} B_{\text {pole }} R_{\mathrm{NS}}^{2}$. Comparing with equation (医) shows that $\alpha(\mathcal{P})$ is proportional to $\mathcal{P}^{1 / p}$, and on dimensional grounds one can write

$$
\alpha(\mathcal{P})=\frac{C^{1 / 2}}{R_{\mathrm{NS}}}\left(\frac{p+1}{p}\right)^{1 / 2}\left(\frac{\mathcal{P}}{\mathcal{P}_{0}}\right)^{1 / p} .
$$

The shape of the field lines, including the radial index $p$, is determined uniquely by the single parameter $C$ which is related to the strength of the current. The poloidal components of eq. (1D) can be integrated to give

$$
B_{\phi}=\frac{\int \alpha(\mathcal{P}) d \mathcal{P}}{R \sin \theta}=\frac{p}{p+1} \frac{\mathcal{P} \alpha(\mathcal{P})}{R \sin \theta} .
$$

Substituting eqs. (21)-(5) into the $\phi$-component of eq. (11) then gives the non-linear equation

$$
p(p+1) F+\left(1-\mu^{2}\right) \frac{\partial^{2} F}{\partial \mu^{2}}=-C F^{1+2 / p}
$$

for the angular factor $F=F(\mu)$.

The solution of eq. (6), including the dependence $p(C)$, is uniquely defined by the parameter $C$ and by the three boundary conditions: $B_{R} \propto F^{\prime}=0$ at $\mu \equiv \cos \theta=0$ (the magnetic equator), $F^{\prime}=$ const $=-2$ at $\mu=1$ (corresponding to a fixed flux density $B_{\text {pole }}$ at the magnetic pole), and $B_{\phi}(\mu=1) \propto F(1)=0$. The solutions are symmetric under $\mu \leftrightarrow-\mu$, with $F(\mu)=1-\mu^{2}$ representing a pure dipole. For each value of $C \leq 0.873$ there are two solutions for $p$. The upper branch connects continuously to the vacuum dipole $C=0, p=1\left(B_{R}, B_{\theta} \propto R^{-3}\right)$; and the lower branch connects to the split monopole $C=0$, $p=0\left(B_{R}= \pm 2 \mathcal{P}_{0} / R^{2}\right)$.

Every twisted force-free magnetosphere has a finite toroidal field

$$
\frac{B_{\phi}(\theta)}{B_{\theta}(\theta)}=\left[\frac{C}{p(1+p)}\right]^{1 / 2} F^{1 / p}(\theta) .
$$


A magnetic field line anchored at polar angle $\theta$ will twist through a net angle

$$
\Delta \phi(\theta)=2 \int_{\theta}^{\pi / 2} \frac{B_{\phi}(\theta)}{B_{\theta}(\theta)} \frac{d \theta}{\sin \theta}
$$

before returning to the stellar surface. Both branches of force-free equilibria connect to form a one-parameter sequence, labeled by the net twist of field lines anchored close to the two magnetic poles, $\Delta \phi_{\mathrm{N}-\mathrm{S}} \equiv \Delta \phi(\theta \rightarrow 0)$. For modest twists $\Delta \phi_{\mathrm{N}-\mathrm{S}} \lesssim 1$ one has

$$
\Delta \phi_{\mathrm{N}-\mathrm{S}} \simeq 2\left(\frac{B_{\phi}}{B_{\theta}}\right)_{\theta=\pi / 2}
$$

The radial index $p$ is a decreasing monotonic function of $\Delta \phi_{\mathrm{N}-\mathrm{S}}$ (Fig. 2). The radial dependence of the magnetic field softens to $\mathbf{B} \propto r^{-2.88}$ when $\Delta \phi_{\mathrm{N}-\mathrm{S}}=1$ radian. The net twist approaches $\Delta \phi_{\mathrm{N}-\mathrm{S}}=\pi$ (one-half turn) in the split monopole limit $(p=0)$. For comparison, a twisted cylindrically symmetric magnetic field pinned in an infinite half-plane expands to infinity after $1 / \sqrt{3}$ turns (Lynden-Bell \& Boily 1994). One example of a twisted, self-similar magnetosphere (corresponding to $\Delta \phi_{\mathrm{N}-\mathrm{S}}=2$ radians) is depicted in Fig. 3 .

These solutions to the force-free equation carry a net helicity

$$
\mathcal{H}_{B}=\int \mathbf{A} \cdot \mathbf{B} d V=\frac{3 \pi}{2}\left(B_{\text {pole }} R_{\mathrm{NS}}^{2}\right)^{2} \sqrt{\frac{C}{p(p+1)}} \int_{0}^{1} d \mu \frac{[F(\mu)]^{2+1 / p}}{1-\mu^{2}} .
$$

This expression reduces to

$$
\mathcal{H}_{B} \simeq \frac{2 \pi}{5}\left(B_{\text {pole }} R_{\mathrm{NS}}^{2}\right)^{2} \Delta \phi_{\mathrm{N}-\mathrm{S}}
$$

for modest twists $\Delta \phi_{\mathrm{N}-\mathrm{S}} \lesssim 1$ radian. The helicity is approximately $\mathcal{H}_{B} \sim B_{\phi} B_{\theta} R^{4}$, and can be expressed in terms of the magnetic energy as $\mathcal{H}_{B} \sim\left(B_{\phi}^{2} R^{3}\right) \times R$ when the twist is moderately large, $B_{\phi} \sim B_{\theta}$. This means that the magnetic energy is minimized at fixed helicity if the twist is concentrated close to the star. Indeed, the current density decreases toward the magnetic axis in these self-similar solutions (as $J(\theta) \sim \theta^{2}$ in the in the case of a modest twist, $\Delta \phi_{\mathrm{N}-\mathrm{S}} \lesssim 1$ ).

Many further properties of these solutions are described in Lyutikov \& Thompson (2001).

\subsection{Effects of Slow Rotation}

We have constructed self-similar solutions to the force-free equation in the infinite volume outside a nonrotating spherical surface. A real neutron star rotates, and its rigidly 
co-rotating magnetosphere has a finite extent, $R \sin \theta \leq c P / 2 \pi \equiv R_{\mathrm{lc}}$ (Goldreich \& Julian 1969). Close to this speed of light cylinder, the rotation will itself cause the field lines to be twisted (Michel 1991; Mestel \& Pryce 1992) - but in a different sense than in the static, twisted magnetosphere. Here we discuss some basic effects of slow rotation, corresponding to an angular velocity of rotation $\Omega \ll c / R_{\mathrm{NS}}$.

The rotational sweeping of the magnetic field lines induces an electric field

$$
\mathbf{E}=-\frac{1}{c}(\boldsymbol{\Omega} \times \mathbf{R}) \times \mathbf{B}
$$

as measured in a background inertial frame. Here $\Omega$ is the angular velocity of the star. The component of $\mathbf{E}$ parallel to $\mathbf{B}$ is cancelled if the closed field lines support a net charge density

$$
\rho=\frac{1}{4 \pi} \nabla \cdot \mathbf{E}=\frac{1}{4 \pi c} \boldsymbol{\Omega} \cdot[-2 \mathbf{B}+\mathbf{R} \times(\nabla \times \mathbf{B})]=\rho_{\mathrm{GJ}}+\rho_{\mathrm{twist}} .
$$

A second term

$$
\rho_{\text {twist }}=\frac{1}{4 \pi c} \boldsymbol{\Omega} \cdot[\mathbf{R} \times(\nabla \times \mathbf{B})] \simeq \frac{1}{c^{2}} \boldsymbol{\Omega} \cdot(\mathbf{R} \times \mathbf{J})
$$

is now present, as compared with the analysis of Goldreich \& Julian (1969). (The second equality in this expression applies to field lines which close well inside the light cylinder.) Thus, a current flowing in a rotating magnetosphere generates a charge density

$$
\frac{\rho_{\text {twist }}}{J / c} \sim \frac{R}{R_{\mathrm{lc}}}
$$

near the magnetic equator, decreasing to

$$
\frac{\rho_{\text {twist }}}{J / c} \sim\left(\frac{R}{R_{\mathrm{lc}}}\right)^{5 / 2}
$$

on the last closed field lines.

The term (14) is easily understood to result from the Lorentz boost of the current $\mathbf{J}$ by the rotational velocity $\Omega \times \mathbf{R}$ of the corotating magnetosphere. In radio pulsar models, it has previously been considered only with respect to the open field lines which may carry a space-charge limited current from the stellar surface (Mestel 1993).

The density of charge carriers needed to support a static twist generally exceeds the standard corotation charge density. At radius $R$ and magnetic co-latitude $\theta$, one has $]$

$$
\left|\frac{J / c}{\rho_{\mathrm{GJ}}}\right| \sim \theta^{2}\left(\frac{R}{R_{\mathrm{lc}}}\right)^{-1}\left(\frac{B_{\phi}}{B_{\theta}}\right)_{\theta=\pi / 2} .
$$

\footnotetext{
${ }^{7}$ We neglect the change in radial index of the field due to the twist.
} 
Polarization of the positive and negative charges flowing along the magnetic field can therefore supply a corotation charge density of either sign. It is also easy to integrate the current flowing along field lines which extend out to a radius $R$; normalizing to the Goldreich-Julian current $I_{\mathrm{GJ}}=\Omega^{2} R_{\mathrm{NS}}^{3} B_{\text {pole }} / 2 c$, one has

$$
\frac{I(R)}{I_{\mathrm{GJ}}} \simeq\left(\frac{R}{R_{\mathrm{lc}}}\right)^{-2}\left(\frac{B_{\phi}}{B_{\theta}}\right)_{\theta=\pi / 2} .
$$

\subsection{Matching of the Twisted Magnetosphere onto Open Field Lines}

On the open field lines, the corotation charge density could be supplied by a space-charge limited flow of ions and electrons from the surface of the neutron star (e.g. Scharlemann, Arons, \& Fawley 1978). In the absence of electron-positron pairs, one necessarily has $J \simeq \rho c$ in such a flow. Because $\rho_{\mathrm{GJ}}=-\boldsymbol{\Omega} \cdot \mathbf{B} / 2 \pi c$ has the same sign above both magnetic poles, a space-charge limited flow with $\rho=\rho_{\mathrm{GJ}}$ implies a current with an opposing sign in the two magnetic hemispheres. By contrast, the closed loops of current flowing through a twisted magnetosphere will maintain a uniform sign near the magnetic axis.

In the absence of pair creation, such a space-charge limited flow would generate, in one hemisphere and close to the magnetic axis, a toroidal magnetic field with the opposite sign to the more global toroidal field. Figure 5 depicts the distribution of toroidal magnetic field in that hemisphere, assuming a geometry for the current flow that is familar from radio pulsar models: the return current (with a sign opposite to the space-charge limited flow) fills a cylindrical sheath near the boundary between open and closed field lines. This return current forces $B_{\phi} \rightarrow 0$ on some cylindrical surface, outside of which $B_{\phi}$ reverses sign due to the global twist (eq. (18)).

Pair creation in the space-charge limited flow allows an entirely different relation between charge and current densities $\rho$ and $J$, because the charges of opposite sign can drift with different speeds:

$$
\rho=e\left(n_{+}-n_{-}\right) \simeq \rho_{\mathrm{GJ}}
$$

whereas

$$
J=e\left(n_{+} v_{+}-n_{-} v_{-}\right) \neq \rho_{\mathrm{GJ}} c .
$$

The total number of charge carriers (of either sign) is greatly amplified, by a factor $N_{\text {pair }} \gg 1$, which we leave as a free parameter. The net current becomes

$$
J \simeq \rho_{\mathrm{GJ}} c+N_{\text {pair }}\left|\rho_{\mathrm{GJ}}\right|\left(v_{+}-v_{-}\right)
$$


As a result, a small electric field parallel to $\mathbf{B}$ is sufficient to reverse the sign of $J$ by inducing a small difference in the speed of positive and negative charges $\left|v_{+}-v_{-}\right| / c \sim N_{\text {pair }}^{-1}$. This allows the current to flow in the same direction on the open field lines as the surrounding closed-field current (and in the opposite direction to the original space-charge limited flow). The difference in Lorentz factors $\left|\Gamma_{+}-\Gamma_{-}\right| / \Gamma \sim N_{\text {pair }}^{-1}$ corresponds to a very modest electrostatic potential drop along the outer magnetic field, $\left|e E_{\|} R\right| \sim\left(\Gamma / N_{\text {pair }}\right) m_{e} c^{2}$. Such a 'reversed' current must be intrinsically time-dependent if the seed charges originate on the surface of the neutron star: otherwise, there will be a build-up of charge above the corotation charge density $\rho_{\mathrm{GJ}}$ in the region where pairs are created.

The implications of this effect for the observed (narrow) distribution of spin periods in the SGR and AXP sources are discussed in $\$$ 6.1. Fast reconnection of these opposing toroidal fields would force a relaxation of the static twist inside the speed of light cylinder, and thus cause a relaxation in the spindown torque.

\section{Resonant Cyclotron Scattering}

A remarkable feature of these twisted, force-free equilibria is that the current currying charges also provide a significant optical depth to resonant cyclotron scattering. A particle of charge $Z e$ and mass $M$ has a resonance frequency $\omega_{c}=Z e B / M c$, and scatters a photon of frequency $\omega$ (incident at angle $\theta_{k B}$ to B) with cross section $\sigma_{\text {res }}(\omega)=\pi^{2}\left(Z^{2} e^{2} / M c\right) \delta\left(\omega-\omega_{c}\right)\left(1+\cos ^{2} \theta_{k B}\right)$ (e.g. Canuto, Lodenquai, \& Ruderman 1971). In a dipole field, the resonance sits at a radius

$$
r_{\text {res }}=\left(\frac{Z e B_{\text {pole }}}{M c \omega}\right)^{1 / 3} f(\theta)=10.5 Z^{1 / 3}\left(\frac{B_{\text {pole }}}{10^{14} \mathrm{G}}\right)^{1 / 3}\left(\frac{M}{m_{e}}\right)^{-1 / 3}\left(\frac{\hbar \omega}{\mathrm{keV}}\right)^{-1 / 3} f(\theta),
$$

in the approximation of static charges. The angular function $f(\theta)=\left(1-\frac{3}{4} \sin ^{2} \theta\right)^{1 / 6}$. The importance of resonant scattering may be characterized by the resonant optical depth $\tau_{\text {res }}$ :

$$
\tau_{\text {res }}(\omega, \theta)=\int n_{Z}(R) \sigma_{\text {res }}\left(\omega-\omega_{c}(R, \theta)\right) d R=\pi^{2} n_{Z} Z e\left(1+\cos ^{2} \theta_{k B}\right) \frac{R_{\text {res }}}{B\left(R_{\text {res }}\right)}\left|\frac{d \ln R}{d \ln B}\right|,
$$

where $n_{Z}$ is the plasma density at the location of the resonance. The shape of the resonant surface becomes more spherical in the presence of a net twist, but only slightly when $\Delta \phi_{\mathrm{N}-\mathrm{S}} \lesssim 1$ (Lyutikov \& Thompson 2001).

The plasma density at the resonance may be estimated using the current density of the self-similar magnetospheric models considered in Section 3 (eqs. (11) and (4)), $J=(c / 4 \pi)\left(B / R_{\mathrm{NS}}\right)[C(p+1) / p]^{1 / 2}\left(\mathcal{P} / \mathcal{P}_{0}\right)^{1 / p}$. Assuming a drift velocity $v^{Z} \leq c$, the particles 
of charge $Z e$ and mass $M$ generate an optical depth satisfying

$$
\begin{aligned}
\left(\frac{v^{Z}}{c}\right) \tau_{\text {res }} & =\varepsilon^{Z} \frac{\pi}{4}\left(1+\cos ^{2} \theta_{k B}\right)\left[\frac{C(1+p)}{p}\right]^{1 / 2} \frac{[F(\theta)]^{1 / p}}{2+p} \\
& =\frac{\pi \varepsilon^{Z}}{4}\left(1+\cos ^{2} \theta_{k B}\right)\left(\frac{p+1}{p+2}\right)\left[\frac{F(\theta)}{F(\pi / 2)}\right]^{1 / p}\left(\frac{B_{\phi}}{B_{\theta}}\right)_{\theta=\pi / 2}
\end{aligned}
$$

(assuming that the particles carry a fraction $\varepsilon^{Z}$ of the current). Resonant scattering (at much lower frequencies) by a Goldreich-Julian current flowing along open magnetic field lines in a dipolar magnetic field has been considered previously by Rajagopal \& Romani (1997).

Two features of the expression (24) deserve to be emphasized. First, the product $\left(v^{Z} / c\right) \tau_{\text {res }}$ equilibrates to a value near unity when the twist is significant, $B_{\phi} / B_{\theta} \sim 1$ at the magnetic equator, independent of the mass and charge of the scatterers, the radius, or the resonant frequency (with the obvious proviso that the resonant radius sits outside the star). Second, the optical depth to scattering vanishes near the magnetic axis, where the equilibrium current density is reduced as the result of the large extent of the field lines (Fig. (1). The angular factor in eq. (24) is $[F(\theta)]^{1 / p} \simeq \sin ^{2} \theta$ for twists $\Delta \phi_{\mathrm{N}-\mathrm{S}} \lesssim 1$ radian $\left(F \simeq 1-\mu^{2}, p \simeq 1\right)$.

Thus the emission leaving the surface of the neutron star near equatorial plane will be strongly scattered at the cyclotron resonance, while at the poles the emission will emerged almost unscattered. This axisymmetric, self-similar model will more accurately describe the magnetospheric structure of an SGR/AXP at larger distances from the star, where the magnetic field lines are anchored in a small portion of the neutron star surface, and their arrangement is less sensitive to irregularities in the surface flux density. As a result, the scattering surface should more closely approximate this simple, axisymmetric form at lower frequencies, and at the electron cyclotron resonance than at the ion resonance(s).

A similar result can be derived in any situation where the magnetic field is strongly sheared, and can be applied to more complicated (e.g. multipolar) magnetic field geometries. The resonant optical depth along a ray is inversely proportional to the gradient of $B$ parallel to the ray,

$$
\tau_{\mathrm{res}}=\pi^{2}(Z e) n_{Z}\left(1+\cos ^{2} \theta_{\mathrm{kB}}\right)\left|\frac{d l}{d B}\right| .
$$

Estimating $(Z e) v^{Z} n^{Z} \sim \frac{1}{2}(c / 4 \pi)|\nabla \times \mathbf{B}|$ (the resonant charges carry half the current), one has

$$
\tau_{\text {res }}\left(\frac{v^{Z}}{c}\right)=\frac{\pi}{8}\left(1+\cos ^{2} \theta_{\mathrm{kB}}\right)|\nabla \times \mathbf{B}|\left|\frac{d l}{d B}\right| .
$$


Strong shearing of the magnetic field corresponds to $|\nabla \times \mathbf{B}||d l / d B| \sim 1$, and implies strong Doppler heating within the scattering layer.

\subsection{Equilibrium Drift Speed}

Current-carrying charges will feel a drag force when they enter a region with a significant flux of photons at the cyclotron energy. In the quiescent state of an SGR (or AXP) source, the drag force acting on the ion component of the current is only a modest fraction of gravity (Appendix B). The minimum drift speed of the upward moving ions at the surface of the star can then be related to the gravitational potential difference which they traverse along a magnetic flux line,

$$
\Delta \phi=\left(1-\frac{R_{\mathrm{NS}}}{R_{\max }}\right) \frac{G M_{\mathrm{NS}}}{R_{\mathrm{NS}}},
$$

through

$$
\frac{v_{i}(\min )}{c}=\left(\frac{2 \Delta \phi}{c^{2}}\right)^{1 / 2} \simeq 0.6\left(1-\frac{R_{\mathrm{NS}}}{R_{\max }}\right)^{1 / 2} .
$$

The drift speed will decrease as the ions climb the potential.

The drag force acting on the electrons is largest at a radius $R \sim 100 \mathrm{~km}$, where their cyclotron energy is in the keV range. The spectral energy density of the radiation field can then be estimated as $U_{\omega} \sim L_{\omega} / 4 \pi R^{2} c$. An electron moving along $\mathbf{B}$ with a particular speed $v_{X}$ will feel a Compton drag force which is perpendicular to $\mathbf{B}$, so that the electron does not gain or lose kinetic energy. In general $v_{X}$ is a modest fraction of the speed of light $c$ and does not equal the drift speed $v_{e}$ of the current-carrying electrons: for example, $v_{X}=\cos \theta_{\mathrm{kB}}$ when the photons stream at a fixed angle $\theta_{\mathrm{kB}}$ with respect to $\mathbf{B}$. To clarify the discussion that follows, we consider only the simplest case where the radiation field is directed perpendicular to $\mathbf{B}$, and $v_{X}=0$.

The component of the Compton draft force parallel to $\mathbf{B}$ can be written as

$$
F_{\|} \sim \Gamma_{e}^{2}\left(\frac{v_{e}}{c}\right) \int \frac{L_{\omega}}{4 \pi R^{2} c} \sigma\left(\omega, \theta_{\mathrm{kB}}=\pi / 2\right) d \omega ;
$$

here $v_{e}$ and $\Gamma_{e}$ are the speed and Lorentz of the electron along B. Substituting the resonant cross section, one finds that this drag force is easily sufficient to slow an electron to sub-relativistic speed, unless it is compensated by an electrostatic force

$$
e E_{\|} \sim \frac{\pi}{4} \Gamma_{e}^{2}\left(\frac{v_{e}}{c}\right)\left(\frac{e^{2}}{m_{e} c^{2}}\right)\left(\frac{L_{\omega_{c}}}{R_{\mathrm{res}}^{2}}\right) .
$$


The measured spectrum of a magnetar is low enough in the optical bands (Hulleman et al. 2000) that the ions feel a negligible Compton drag force at the same radius that the electrons resonantly scatter off keV photons. Thus, after exiting the electron resonance layer, the ions (which we take to be protons) will have a minimum kinetic energy

$$
\left(\Gamma_{p}-1\right) m_{p} c^{2} \sim e E_{\|} R_{\mathrm{res}}
$$

Now the assumption of a steady current flow, combined with charge neutrality $\left(n_{p}=n_{e}\right)$, leads to the requirement that the ratio of drift speeds $v_{e} / v_{p}$ maintain a constant value $\varepsilon_{e} / \varepsilon_{p}$ within the resonance scattering layer. (As before, $\varepsilon_{i}$ is the fraction of the current carried by particle species $i$. Notice that only a tiny charge separation $\left|n_{p}-n_{e}\right| / n_{e}=O\left(10^{-12}\right)$ is needed to maintain the electrostatic field (30).) In the simplest case where $v_{e}=v_{p}$, one deduces

$$
\frac{\Gamma_{\mathrm{eq}}-1}{\Gamma_{\mathrm{eq}}^{2}\left(v_{\mathrm{eq}} / c\right)} \sim \frac{\pi e^{2}}{4 m_{p} m_{e} c^{4}} \frac{\left(\omega L_{\omega}\right) \omega_{c}}{R_{\mathrm{res}} \omega_{c}}
$$

for the equilibrium drift speed (Lorentz factor) within the resonant scattering layer. Re-expressing the resonant radius $R_{\text {res }}$ in terms of the surface polar (dipole) field, this equation becomes

$$
\frac{\Gamma_{\mathrm{eq}}-1}{\Gamma_{\mathrm{eq}}^{2}\left(v_{\mathrm{eq}} / c\right)} \sim 0.9\left[\frac{\left(\omega L_{\omega}\right)_{\omega_{c}}}{10^{35} \mathrm{erg} \mathrm{s}^{-1}}\right]\left(\frac{\hbar \omega_{c}}{\mathrm{keV}}\right)^{-2 / 3}\left(\frac{B_{\mathrm{pole}}}{10^{14} \mathrm{G}}\right)^{-1 / 3}\left(\frac{R_{\mathrm{NS}}}{10 \mathrm{~km}}\right)^{-1}
$$

One observes that mildly relativistic motion of the charge carriers is implied if the X-ray luminosity of the source is $\sim 10^{35} \mathrm{erg} \mathrm{s}^{-1}$. The left side of eq. (33) has a maximum value of $\simeq 0.3$ at $v_{\text {eq }} / c \simeq 0.8$. The current must, as a result, be dominated by ions when $L_{X}$ significantly exceeds $\sim 10^{35} \mathrm{erg} \mathrm{s}^{-1}$.

\section{Implications for X-ray Spectra and Pulse Profiles}

We have described the basic properties (magnetic field geometry, optical depth to resonant scattering) of a class of twisted, self-similar solutions to the force free equation. In this section, we show how several observed properties of the SGR and AXP sources connect directly with this model, which provides a promising framework for synthesizing the two populations. 


\subsection{Surface Heating (vs. Volumetric Heating)}

Transport of a magnetic field in the deep crust and core of a magnetar will result in dissipation both inside and outside the star (TD96). The heat released in the core (through frictional heating and beta reactions) is converted largely to neutrinos, and will be conducted to the surface with a delay of one year or longer if the surface temperature is $\sim 0.5 \mathrm{keV}$. Non-potential distortions of the magnetic field outside the star are converted much more efficiently to visible electromagnetic radiation (X-rays). Changes in the external magnetic field will, as a result, induce equally sudden changes in the source brightness (e.g. SGR flares).

In the present context, where we are interested in a smooth shearing deformation of the magnetic field, the dissipation outside the star takes two principal forms: impact of the current-carrying charges on the stellar surface; and resonant Comptonization of this surface X-ray flux by the magnetospheric currents. The surface heating provides a minimal radiative output of a magnetar, which is increased by a modest factor as the X-rays undergo

multiple scattering (\$5.4). When $B_{\text {dipole }}$ (multiplied by the irradiated fraction of the stellar surface) is $\sim 10^{14} \mathrm{G}$, we find that the surface $\mathrm{X}$-ray flux is, by coincidence, comparable to the equilibrium flux powered by ambipolar diffusion of a strong internal magnetic field (TD96; Heyl \& Kulkarni 1998). The angular pattern of the surface radiation can be expected to differ significantly from models of passively cooling magnetars; the implications for pulse fractions are discussed in $\$ 5.5$.

Both positive and negative charges are needed to cancel the space charge outside the neutron star. Let us consider the simplest case where the surface has a light element composition, and positive ions (e.g. hydrogen, helium, or carbon) can be emitted thermionically (Thompson et al. 2000). The electric field which lifts an ion through the gravitational potential of the star is many orders of magnitude smaller than the field induced by a bare space charge $\rho \sim J / c$. A first estimate of the minimum power consumed by the current is obtained by summing the gravitational potential energy of the ions falling onto the cathode, with the (approximately equal) electrostatic energy picked up by returning electrons at the anode, where the ions are accelerated. The contribution to the power from an infinitesimal current $J\left(R_{\mathrm{NS}}\right) d A$ originating in a surface element $d A$ is then $d L_{X}=2\left(G M_{\mathrm{NS}} m_{p} / R_{\mathrm{NS}}\right)\left(1-R_{\mathrm{NS}} / R_{\max }\right) J\left(R_{\mathrm{NS}}\right) d A / e$. Here $R_{\max } / R_{\mathrm{NS}}=[F(\theta) / F(\pi / 2)]^{-1 / p}$ is the maximum radius attained by a field line anchored at polar angle $\theta$. One observes that the heating rate is strongly inhomogeneous over the surface of the star, even under the assumption of a cylindrically symmetric and self-similar magnetosphere. The current density varies across the stellar surface, as does the gravitational potential shift along magnetic flux lines of different lengths. 
Integrating over the surface of the star, we find

$$
\begin{aligned}
L_{X} & =\frac{B_{\text {pole }} G M_{\mathrm{NS}} m_{p} c}{2 e} \frac{p F(\pi / 2)}{2+p}\left(\frac{B_{\phi}}{B_{\theta}}\right)_{\theta=\pi / 2} \\
& \simeq 3 \times 10^{35}\left(\frac{B_{\text {pole }}}{10^{14} \mathrm{G}}\right)\left(\frac{M_{\mathrm{NS}}}{1.4 M_{\odot}}\right)\left(\frac{B_{\phi}}{B_{\theta}}\right)_{\theta=\pi / 2} \quad \operatorname{erg~s}^{-1} .
\end{aligned}
$$

In this self-similar model, the rate of surface heating is close to the persistent X-ray luminosity of the SGR and AXP sources if $B_{\text {pole }} \times\left(B_{\phi} / B_{\theta}\right)_{\theta=\pi / 2} \sim 10^{14} \mathrm{G}$. The heating rate is too high if the magnetosphere is strongly twisted and $B_{\text {pole }} \sim 10^{15} \mathrm{G}$, but in reality the twist could be more localized - e.g. around the magnetic poles.

There is an inefficiency in driving this form of dissipation by ambipolar diffusion of the core magnetic field: the total radiative output of an AXP or SGR is $\sim 10^{47}$ ergs over its $\sim 10^{4}$ year lifetime, but the total energy radiated in neutrinos is some $\sim 30$ times higher. The minimal (r.m.s.) magnetic field needed to power the observed activity is $\sim 10^{15} \mathrm{G}$, which increases by a factor $\sim 5$ to allow rapid diffusion of the field through the core. $y^{\circ}$ In this regard, the Sun provides a rough analog: distortions of the magnetic field outside its photosphere are converted efficiently to non-thermal radiation (Solar flares). However, only a tiny fraction of the total energy stored in the Solar magnetic field is actually released in its exterior: only one part in a thousand of the bolometric Solar output is radiated through the chromosphere and corona. In a magnetar, motions of the external magnetic footpoints are driven gradually by diffusive processes in the deep crust and core, and also suddenly by brittle fractures of its crust. We focus in this paper on smooth shear deformations of the external magnetic field, but the possibility remains that a magnetar will also experience an approximate analog of 'microflaring' on the Sun: the Hall electric field will drive high-frequency dislocations of its crust $(\lambda \lesssim 0.1 \mathrm{~km})$, which couple efficiently to magnetospheric Alfvén waves (TD96).

\subsection{Decay of the External Twist}

The preceding result (eq. 34]) also allows a simple estimate of the maximum time for the external twist to dissipate completely, in the absence of further sub-surface motions.

\footnotetext{
${ }^{8}$ These fields are easily strong enough to break the neutron star's rigid crust; but observations of magnetic white dwarfs demonstrate the existence of stable magnetostatic equilibria even in perfectly fluid, degenerate stars. When the magnetic field is pinned by a rigid crust, transport of the field will force the build-up of shear stresses in the crust to a (small) fraction of the bulk magnetostatic stresses; see Thompson \& Duncan (1995), (2001).
} 
The energy of a twisted magnetosphere exceeds the energy of a pure dipole with the same polar flux density,

$$
\frac{E_{B}}{E_{B}(\text { dipole })}=\frac{3}{2(p+1)} \int_{0}^{1} d \mu\left(\frac{d F}{d \mu}\right)^{2}>1
$$

and can be approximated by

$$
\frac{E_{B}}{E_{B}(\text { dipole })}=1+0.17 \Delta \phi_{\mathrm{N}-\mathrm{S}}^{2}
$$

for $\Delta \phi_{\mathrm{N}-\mathrm{S}} \lesssim 1$. This works out to

$$
E_{B}-E_{B}(\text { dipole })=1.4 \times 10^{44} \Delta \phi_{\mathrm{N}-\mathrm{S}}^{2}\left(\frac{B_{\text {pole }}}{10^{14} \mathrm{G}}\right)^{2}\left(\frac{R_{\mathrm{NS}}}{10 \mathrm{~km}}\right)^{3} \quad \mathrm{erg},
$$

for twists $\lesssim 1$ radian. The decay time for the global twist is then

$$
t_{\text {decay }}=\frac{E_{B}-E_{B}(\text { dipole })}{L_{X}}=40 \Delta \phi_{\mathrm{N}-\mathrm{S}}^{2}\left(\frac{L_{X}}{10^{35} \mathrm{erg} \mathrm{s}^{-1}}\right)^{-1}\left(\frac{B_{\text {pole }}}{10^{14} \mathrm{G}}\right)^{2}\left(\frac{R_{\mathrm{NS}}}{10 \mathrm{~km}}\right)^{3} \quad \text { yr. }
$$

very similar to the estimate made in Thompson et al. (2000).

The amount of surface heating would be reduced by a factor $\gamma_{e}\left(m_{e} / m_{p}\right)\left(c^{2} / G M_{\mathrm{NS}}\right)$ and the lifetime of the external twist would be increased correspondingly — if the current were carried by relativistic pairs of energy $\gamma_{e} m_{e} c^{2}$.

\subsection{The Ion Cyclotron Resonance and Higher Multipoles}

In this self-similar model, the ion component of the current will generate a comparable optical depth to the electron component in a self-similar magnetosphere. The optical depth to resonant scattering is independent of the net mass or electrical charge, excepting the factor $\varepsilon^{Z}\left(v^{Z} / c\right)^{-1}$ (eq. 24.) Indeed, the cyclotron energy of an ion of charge $+Z e$ and mass $A m_{n}$ in a magnetic field $B$ is $\hbar \omega=6.3(Z / A)\left(B / 10^{15} \mathrm{G}\right) \mathrm{keV}$. Although this resonance sits at X-ray frequencies in magnetar-strength fields, the resonant radius (22) is $\sim 10$ times smaller for ions than electrons (or positrons).

The heavier particles will resonantly scatter a more restricted range of frequencies (below their surface cyclotron frequency). This means that higher magnetic multipoles will leave a more direct imprint in the pulse profile through resonant ion scattering, than electron scattering. Notice also that higher multipoles will increase the surface field strength over the polar dipole value, and hence increase the range of frequencies which resonantly 
scatter close to the star. The pulse profile of the August 27 giant flare provides direct evidence for the presence of higher magnetic multipoles in SGR 1900+14: four sub-pulses of a large amplitude appeared during the intermediate portion of the burst, which repeated coherently with the 5.16 spin period (Feroci et al. 2001; Thompson \& Duncan 2001).

\subsubsection{Surface Stopping of Magnetospheric Charges and Ion Cyclotron Emission}

The surface of a magnetar will be impacted by ions or electrons, depending on the sign of the electrical current. The characteristic kinetic energy of an ion is its gravitational binding energy to the star, $E_{i} \sim G M_{\mathrm{NS}}\left(A m_{p}\right) / R_{\mathrm{NS}}=200 A \mathrm{MeV}$. If the ion flow contains a significant proton component, then a layer of hydrogen will form at the cathode surface. The downward-moving protons will be stopped by $p-p$ collisions at a depth corresponding to an electron column $\sigma_{T} N_{e} \simeq 30$.

At the anode surface (where the electrostatic field accelerates the ions of mass $A$ and charge $Z$ upward), the downward-moving electrons will be pushed to relativistic energies $E_{e} \simeq 200(A / Z) \mathrm{MeV}$. In surface fields $B \gtrsim 10^{15} \mathrm{G}$, these particles are not effectively stopped by long-range Coulomb interactions, because the background electron gas is effectively one-dimensional (e.g. Nelson, Salpeter, \& Wasserman 1993). The electrons can be stopped by spallation of protons from ions (Thompson et al. 2000),

$$
e^{-}+(Z, A) \rightarrow e^{-}+(Z-1, A)+p
$$

If the anode surface is composed of light ions, e.g. helium or carbon, then the electrons are stopped at a depth corresponding to $N_{e} \sigma_{T} \sim 10^{4}$. This stopping mechanism has the interesting consequence that each electron will create an unbound proton. If the downward-moving electrons and upward-moving ions contribute equally to the current density, the proportions of the ion current carried by the protons and the heavier (spallated) ions are also approximately equal.

We point out here the interesting possibility that ion cyclotron emission line may be observed in magnetars whose surfaces are heated by magnetospheric charges. At both ends of the circuit, a significant fraction of the kinetic energy of the impacting charges is transferred directly to the ions. This spectral feature is easiest to detect if the surface magnetic field lies in the range $B_{S}=(0.2-2) \times 10^{15}(A / Z) \mathrm{G}$, so that the ion cyclotron resonance sits between 1 and $10 \mathrm{keV}$. Phase-resolved spectroscopy of magnetar candidates

\footnotetext{
${ }^{9}$ Using the cross section of Schaeffer, Reeves, \& Orland (1982) for $e^{-}+{ }^{12} C \rightarrow e^{-}+{ }^{11} B+p$.
} 
could, for this reason, provide a direct measure of the fraction of the thermal emission powered by magnetospheric currents.

The nature of the radiative transport near the ion cyclotron resonance is substantially different in 'naked' magnetars with persistent magnetospheric currents, than in accreting neutron stars. The density of returning, current-carrying ions is almost constant above the surface of a magnetar - in contrast with an accreting neutron star, onto which the infalling matter gradually settles. The resonant optical depth (eq. [23]) is proportional to the local density of scattering charges (rather than to the column density as with non-resonant scattering). This means that $\tau_{\text {res }}$ has a strong spike just above the surface of an accreting neutron star, but remains approximately constant above the surface of an isolated magnetar with a non-potential magnetic field. In the accreting case, the result is the formation of an absorption feature at an X-ray cyclotron resonance (Zane, Turolla, \& Treves 2000). In the magnetar case, by contrast, the kinetic energy lost by the impacting charges will raise the temperature in a thin surface layer - without a large cyclotron opacity developing outside that layer. This allows an emission feature to form at a cyclotron resonance. (Nelson et al. 1995 have demonstrated this effect for electron cyclotron scattering in a $\sim 10^{12} \mathrm{G}$ magnetic field, at the surface of a neutron star which is impacted by ions moving at the free fall speed.)

\subsection{Resonant Compton Heating}

Multiple scattering at the cyclotron resonance will redistribute photons in frequency. This effect has some similarities with Comptonization in an accretion flow (Blandford \& Payne 1981), except that resonant scattering of a photon of a given frequency occurs only at a particular resonant surface: space and energy diffusion are now directly coupled. Photons which are backscattered return to a region where their energy lies below the local cyclotron resonance, and will undergo subsequent resonant scatterings.

The optical depth to resonant scattering by charges moving with drift speed $v$ is $\simeq(v / c)^{-1}\left(B_{\phi} / B_{\theta}\right)_{\theta=\pi / 2}$ at the magnetic equator (eq. 24]). Let us first consider the case of electron scattering. Resonant scattering by $\sim \mathrm{keV}$ photons at a radius $R / R_{\mathrm{NS}} \sim 10\left(B_{\text {pole }} / 10^{14} \mathrm{G}\right)^{1 / 3}$ maintains a mildly relativistic electron drift speed when $\omega_{c} L_{\omega_{c}}=10^{35} \mathrm{erg} \mathrm{s}^{-1}$ (\$4.1). In addition, the product $\left(v_{e} / c\right) \tau_{\text {res }}$ has no explicit frequency dependence below an energy $\hbar \omega \sim m_{e} c^{2}$, and above the very low cyclotron frequency at the speed of light cylinder $\omega_{c, e} / 2 \pi \sim 10^{8} \mathrm{~Hz}$.

In spite of the complicated geometry of the magnetosphere, we can draw a remarkably 
simple conclusion: the product of the mean frequency shift per scattering $\Delta \omega / \omega$ and the number of scatterings is $O(1)$ when the twist angle $\Delta \phi_{N-S} \sim 1$. As a result, multiple resonant scattering of thermal X-ray photons, within a self-similar twisted magnetosphere, provides a simple mechanism for generating a high energy tail to the X-ray spectrum. In the case of electron cyclotron scattering, this high energy tail will extend up to a frequency $\hbar \omega_{\text {max }} \sim \frac{1}{2} m_{e} v_{e}^{2} \gtrsim 100 \mathrm{keV}$, where the increase in frequency due to the Doppler effect is balanced by the Compton recoil.

At a fixed spectral intensity, the hardness of the high energy tail will increase with the number of scatterings and, hence, with the net twist imparted to the external magnetic field. However, this relation between spectral hardness and magnetospheric current can be compensated by changes in spectral intensity, for the reason that the drift speed of the electrons varies as a complicated function of $L_{\omega_{c}}$ when $\left(\omega L_{\omega}\right)_{\omega_{c}} \lesssim 10^{35} \mathrm{erg} \mathrm{s}^{-1}$ (\$1.1). The often measured ratio of power-law to thermal intensities should also be interpreted with caution in this situation: if the thermal seed is generated primarily by surface heating, then its spectrum will not be a pure black body, and a spectral decomposition into black body and power-law components will not accurately measure the relative luminosities carried by the surface and magnetospheric components.

\subsubsection{Scattering at the Ion Cyclotron Resonance}

The optical depths to ion and electron cyclotron scattering are comparable in this self-similar model. More generally, if the surface magnetic field is strongly sheared, then a the product of the resonant optical depth and the ion drift speed satisfies $\tau_{\text {res }}\left(v^{Z} / c\right) \sim 1$ across a resonant layer (\$田).

However, multiple scattering by ions is different in three important respects. First, as discussed in Section 5.3, it is sensitive to the presence of higher-order multipoles in the magnetic field. Second, the upper cutoff to the non-thermal spectral tail is not set by the Compton recoil, but instead by the surface cyclotron frequency,

$$
\hbar \omega_{\max }=\frac{\hbar \omega_{c, i}\left(R_{\mathrm{NS}}\right)}{\gamma_{i}\left(1-v_{i} / c\right)} \simeq \frac{30}{\gamma_{i}\left(1-v_{i} / c\right)}\left(\frac{Z}{A}\right)\left(\frac{B}{100 B_{\mathrm{QED}}}\right) \quad \mathrm{keV}
$$

In this expression, the Doppler factor $\gamma_{i}^{-1}\left(1-v_{i} / c\right)^{-1}$ takes into account the (upward) motion of the ions with speed $v_{i}$. Even if the ions are moving sub-relativistically, their kinetic energy $\frac{1}{2}\left(A m_{p}\right) v_{i}^{2}$ will greatly exceed $\hbar \omega_{c, i}$. A non-thermal spectrum extending above $20 \mathrm{keV}$ can be produced by resonant ion scattering if the surface field is stronger than $\sim 3 \times 10^{15} \mathrm{G}$, i.e., if higher multipoles are present in the magnetic field. Indeed, higher multipoles are almost certainly present in the SGR sources (Feroci et al. 2001). 
Third, a photon has a much larger probability of being backscattered directly at the neutron star within the ion corona $(R \sim 10-20 \mathrm{~km})$ than in the electron corona $(R \sim 50-100 \mathrm{~km})$. Multiple ion scattering is most effective if ions of different charge/mass ratios $Z / A$ are present. For example, protons and heavier ions will carry comparable fractions of the current if the electron charge carriers returning to the neutron star surface are stopped by spallating protons from the ions, as is expected in magnetic fields stronger than $\sim 10^{15} \mathrm{G}$ (\$.3.1). The heavier ions, having a lower charge/mass ratio $Z / A \simeq \frac{1}{2}$, will resonantly scatter X-rays closer to the star. This means that the resonance condition $\omega=\omega_{c, i} / \gamma_{i}\left(1 \mp v_{i} / c\right)$ can be satisfied for both outward-moving and inward-moving photons if the backscattering is by the protons at a larger radius. The inner and outer resonant surfaces are converging in a region where the ions are streaming outward from the star (and losing kinetic energy in its gravitational potential) and an extended high-energy spectral tail will result.

\subsubsection{Comparison with the SGR and AXP Sources.}

The spectra of some AXPs are inferred to have very soft power-law components (photon index $\sim-4$ ). These soft spectra may be explainable in terms of passive radiative transport through the surface of a neutron star with a $\sim 10^{14} \mathrm{G}$ magnetic field (Özel 2001; Ho \& Lai 2001; Lai \& Ho 2001). It should, nonetheless, be kept in mind that the spectrum of one AXP is much harder (the photon index is -2.5 for 1E 1048.1-5937; Oosterbroek et al. 1998); and the actively bursting SGRs have even harder spectra (photon indices -2.2 ; Hurley 2000, and references therein). Multiple resonant scattering provides a mechanism for generating the full observed range of power-law indices: the softest AXP spectra correspond to external magnetic fields which carry relatively weak electrical currents, and the hardest SGR spectra to magnetospheres which are strongly twisted.

It is interesting to note that $t_{\text {decay }}$ (eq. [38]) is comparable to the time since the 5 March 1979 giant flare if the polar field $\mathbb{U}$ is $\simeq 10^{14} \mathrm{G}$. The relatively soft power-law spectrum of SGR 0526-66 (Kulkarni et al. 2001) may therefore have an explanation in terms of the decay of the external magnetic shear following the flare.

As we have discussed, the atmosphere of a magnetar can be expected to have more than one scattering corona. The observations do not yet allow us to distinguish between the case where the coronal heating is dominated by electron cyclotron scattering, versus ion cyclotron scattering (or non-resonant $e^{ \pm}$scattering) closer to the star. Only scattering

\footnotetext{
${ }^{10}$ To be more precise, the dipole field in the absence of any external twist.
} 
by electrons can create a non-thermal spectrum extending up to $\sim m_{e} c^{2}$ if the charges are mildly relativistic (\$4.1). For this reason, high energy observations of persistent SGR spectra (between 20 and $500 \mathrm{keV}$ ) would provide a clear discriminant between coronal heating dominated by electron vs. ion scattering.

More detailed calculations of the distribution of scatterings and frequency shifts are under way.

\subsection{Pulsations in the Persistent Emission}

The Anomalous X-ray Pulsars have been proposed to be either magnetars powered by active field decay (TD96), or passively cooling neutron stars with $\sim 10^{14}$ G magnetic fields (Heyl \& Hernquist 1997). In addition to the constancy of the measured X-ray flux, these models may also be distinguished by the pattern of the emergent X-ray pulsations. The X-ray spectra and pulse profiles produced by passively cooling neutron stars have been calculated by Psaltis, Özel, \& DeDeo (2000) and Özel (2001), taking into account the gravitational deflection of the photons, the angular variation of the opacity, and the interchange between the two X-ray polarization modes in the outer atmosphere. They find that the pulse fractions are less than those measured in two AXPs (e.g. Table 2 in Kaspi et al. 2001), even in the case of cooling through a single, localized hotspot. However, as we now describe, bombardment of the neutron star surface by persistent currents, and re-scattering of the X-rays in the magnetosphere, can lead to significantly different pulse profiles.

It should first be emphasized that the angular distribution of the surface X-ray flux depends on the relative proportions of the flux carried by the extraordinary polarization mode (the E-mode) and the ordinary mode (the O-mode). The O-mode radiation is strongly beamed at the surface of a neutron star, because its scattering opacity scales as $\sin ^{2} \theta_{k B}$ at frequencies well below the electron cyclotron frequency (Basko \& Sunyaev 1975). However, a large fraction of the energy flux across the surface of a passively cooling neutron star is carried by the E-mode, whose opacity is more isotropic.

At the surface of a magnetar, the beaming of the O-mode radiation is most important when the X-ray flux is generated by particle heating. In that case, most of the surface flux will emerge in the O-mode and, in addition, the heating may be localized at a small hot spot, which allows the formation of a narrow cone of radiation.

\footnotetext{
${ }^{11}$ As before, $\theta_{k b}$ is the angle between the background magnetic field and the photon wavevector.
} 
The opacity of the E-mode is suppressed in an ultrastrong magnetic field by a factor $\left(m_{e} c \omega / e B\right)^{2}=7 \times 10^{-7}(\hbar \omega / \mathrm{keV})^{2}\left(B / 10^{14} \mathrm{G}\right)^{-2}$. Impacting protons will be stopped by $p-p$ collisions at a depth corresponding to $N_{e} \sigma_{T} \sim 30$ - well above the position of the E-mode photosphere but, generally, below the O-mode photosphere.

\subsubsection{Cyclotron Scattering in the Magnetosphere}

Resonant cyclotron scattering will strongly modify the angular pattern of the X-ray flux emerging from a twisted, current-carrying magnetosphere. This reprocessing will occur at the electron cyclotron resonance at a large distance of $\sim 50-100 \mathrm{~km}$, where gravitational bending of the photon trajectories can be largely neglected. Reprocessing will also occur at the ion cyclotron resonance closer to the star, at $10-20 \mathrm{~km}$.

Three effects are important here:

1. The optical depth to resonant scattering is a strong function of angle in a self-similar, twisted magnetosphere (Fig. (1)). Even when the twist is large, $\Delta \phi_{\mathrm{N}-\mathrm{S}} \sim 1$ radian, and the optical depth exceeds unity at the magnetic equator, the resonant scattering surface maintains two holes of solid angle $\sim 2 \mathrm{Sr}$, centered at the two magnetic poles. This effect will increase the relative X-ray flux emerging along the magnetic axes.

2. The scattered cyclotron radiation is beamed parallel to $\mathbf{B}: d \sigma_{\text {res }} / d \Omega \propto 1+\cos ^{2} \theta$ in the rest frame of the scattering charge. A signature of this effect is the appearance of a fourth-order harmonic in the pulse profile. Note also that the degree of beaming can be greatly enhanced by the bulk motion of the charge carriers in an electron-ion current. Consider, for example, an optically thick scattering screen which moves at uniform speed $v$ along the magnetic field. The intensity of the radiation emerging from the screen is concentrated at an angle $\theta=\cos ^{-1}(v / c)$ with respect to $\mathbf{B}$.

3. The resonant surface is aspherical, with a cross-sectional area that is larger when viewed along the magnetic equator, than from one of the magnetic poles. This effect will enhance the relative flux propagating to infinity along the magnetic equator.

Each of these effects can, by themselves, generate one or two sub-pulses - depending on the orientation of the rotation and magnetic axes with respect to each other and to the line of sight. Calculations of the resultant pulse profiles are underway.

Even if the magnetic field were axisymmetric in the absence of electrical currents, deformations of the neutron star crust which generate the currents could have azimuthal structure. Resonant scattering by non-axisymmetric currents therefore provides an 
additional source of structure in the X-ray pulse profile.

\subsection{Implications for Giant Flare Mechanism}

There are two generic possibilities for the production of the giant flares of the SGRs, in the framework of our model (Thompson \& Duncan 1995, 2001; Woods et al. 2001a). First, a giant flare may result from a sudden change (unwinding) in the internal magnetic field. In this case, a twist is implanted into the magnetosphere. A large-scale displacement of the crust probably requires the formation of a propagating fracture, close to which the magnetic field is strongly sheared. An attractive mechanism for powering the flare involves the repeated excitation and relaxation of a high current density around the fault. The energy stored in a twisted internal field, which is available for sudden release, can be related

to the limiting strain $\psi_{\max }$ of the crust, $\Delta E_{B}=1 \times 10^{46}\left(\psi_{\max } / 0.01\right)^{2}\left(B_{\text {pole }} / 10^{14} \mathrm{G}\right)^{-2} \mathrm{erg}$ (Thompson \& Duncan 2001). In the aftermath of the flare, the magnetic field will retain a more smoothly distributed component of the shear. According to this first scenario, the X-ray spectrum should be harder when the X-ray flux has returned to the pre-burst value, but the pulse profile may be simpler as the result of multiple cyclotron scattering.

Alternatively, giant flares may involve a sudden relaxation in the twist outside the star, without the impetus of sudden subsurface motions, in close analogy with Solar flares. This requires that the external magnetic shear build up gradually, and that the outer crust of the neutron star is deformed plastically by internal magnetic stresses. This mechanism has the advantage that the energy stored in the external twist need not be limited by the tensile strength of the crust, but instead by the total external magnetic field energy. From eq. (37), one infers that a polar dipole field of $\sim 3 \times 10^{14} \mathrm{G}$ is needed to power a flare of energy $\sim 10^{45}$ erg through the sudden relaxation of an external twist $\Delta \phi_{\mathrm{N}-\mathrm{S}} \sim 1$ radian. In this second scenario, the X-ray spectrum can be expected to soften following the burst, as the magnetosphere becomes more transparent to cyclotron scattering. These differences in post-flare behavior may serve to distinguish between the two possibilities.

We now discuss four pieces of observational evidence which bear upon this question. Although three seem to support the first hypothesis, the evidence is mixed. The most consistent interpretation is, perhaps, that the giant flares involve a redistribution of current in the magnetosphere, which decreases the magnetic shear locally while at the same time increasing the global twist.

1. The pulse profile of SGR $1900+14$ changed dramatically following the August 27 giant flare, simplifying to a single sinusoidal pulse from 4-5 sub-pulses (Woods et al. 2001a). 
This change has persisted even after the persistent X-ray flux returned to the baseline maintained before the flare (Woods et al. 2001a). This decoupling between the pulse profile and the total X-ray flux from SGR 1900+14, provides evidence that the energy source for the persistent emission is concentrated close to the star, inside the region where the pulse profile is established. Multiple scattering of $2-10 \mathrm{keV}$ photons at the electron cyclotron resonance will cause such a change in pulse profile, if the magnetic field is predominantly dipolar at a radius of $\sim 100 \mathrm{~km}$, and is a natural consequence of a twisting up of the external field during the flare. By contrast, in the second model, the simplified pulse profile would require the elimination of a non-axisymmetric component of the current during the flare. This is more difficult to arrange close to the star, where X-rays can be scattered at the ion cyclotron resonance.

2. Both giant flares (on 5 March 1979 and 27 August 1998) were initiated by a very intense $\sim 0.2-0.5$ s pulse of hard X-rays and gamma rays (Mazets et al. 1979, 1999; Hurley et al. 1999a; Feroci et al. 1999), during which the bulk of the flare energy was probably deposited. This timescale is much longer than the light-crossing time of the central magnetosphere (where the energy in the twisted magnetic field is concentrated); but is similar to the time for a $\sim 10^{15} \mathrm{G}$ magnetic field to rearrange material in the deep crust and core of a neutron star across several kilometers. In the second model, a question of principle also arises as to how a very gradual build up of the external shear (over the estimated interval of $\sim 100$ years between giant flares in any one flare source) could lead to the sudden release of external magnetic energy on a timescale that is some 10 orders of magnitude shorter - without being initiated by a sudden yield or fracture in a rigid component of the star.

3. The persistent spectrum of SGR $1900+14$ softened measureably after the 27 August giant flare: the best-fit spectral index (pure power law) softened from $-1.89 \pm 0.06$ to $-2.20 \pm 0.05$ (Woods et al. 1999a). (Including a black body component, the best-fit index softened dramatically from $-1.1 \pm 0.2$ to $-1.8 \pm 0.2$.) This behavior is not consistent with a twisting up of the outer magnetosphere if the measured spectrum provides a fair measure of the angle-averaged spectrum and, in addition, if the non-thermal continuum is generated by resonant electron cyclotron scattering at $R \sim 50-100 \mathrm{~km}$. However, we have seen that regions of strong localized magnetic shear close to the star provide an alternative location for the source of this continuum - through e.g. resonant ion scattering (泪, 5.4). The measured spectral change in SGR 1900+14 may therefore point to a more 'compact' scattering corona, within which the current relaxed even while it increased on more extended field lines following the flare.

4. Both giant flares were followed by multiple repeat bursts of $\mathrm{a} \sim 1-7 \mathrm{~s}$ duration 
(Golenetskii et al. 1987; Ibrahim et al. 2001) which is intermediate between the flares ( 200 - 400 s) and the much more common short SGR bursts ( $0.1 \mathrm{~s}:$ Gogus et al. 2001). These intermediate bursts released less than one percent of the energy of the giant flares, and most likely represent mild 'aftershocks' of the larger events. This behavior is possible to describe, in our axisymmetric magnetospheric model, if the release of energy is gated by the rigidity of the crust. The case of the 29 August 1998 burst from SGR 1900+14 - which had a similar peak luminosity to the pulsating tail of the August 27 flare but a duration 100 times shorter - is particularly instructive. Evidence for a trapped fireball comes from both bursts: from the shape of the declining light curve in the August 27 flare (Feroci et al. 2001); and from the presence of an extended faint, pulsating tail which followed the bright component of the August 29 burst (Ibrahim et al. 2001). This faint tail had a very hard spectrum which softened with time, and can be explained by the compression and heating of a small patch of the neutron star surface during the preceding burst. The high peak flux and short duration of the August 29 burst require that the magnetically confined

fireball had a planar geometry, so that it cooled rapidly in one direction without decreasing significantly in area. The simplest interpretation here is that the August 29 burst involved a mild slippage of the crust along the same fault line which powered the preceding giant flare (Ibrahim et al. 2001).

\subsubsection{Large-Amplitude Pulsations in the Giant Flares}

Within the 27 August 1998 flare itself, the X-ray flux showed pulsations of a very large amplitude, during all but the first 40 seconds (Hurley et al. 1999a; Feroci et al. 1999; Mazets et al. 1999). These pulsations repeated coherently at the 5.16-s spin period of the star, and the pulse profile maintained a complicated 4-peaked pattern during the intermediate portion of the burst (Feroci et al. 2001), which gradually simplified into a single pulse at the very end (Woods et al. 2001a). The 25-100 keV light curve recorded by Ulysses showed a large asymmetry between the first and second halves of the pulse profile, which was absent in the higher energy 40-700 BeppoSAX GRBM light curve (Feroci et al. 2001). The previous 5 March 1979 giant flare also showed large amplitude pulsations (Mazets et al. 1979). This narrow collimation of the X-ray flux, combined with the quasi-thermal flare spectrum, indicates a large angular variation in the optical depth.

The 'trapped fireball' model developed in Thompson \& Duncan (1995) has been successfully tested against the August 27 flare data in two respects (Feroci et al. 2001). After smoothing over the 5.16-s pulsations, the flare light curve can be well fit by the contracting surface of a magnetically confined $e^{ \pm}$fireball. In addition, the saturation of the 
best-fit X-ray temperature at $\sim 12 \mathrm{keV}$ during the last $\sim 350$-s of the burst is consistent with the freeze-out of photon splitting in a Comptonizing atmosphere, as predicted by Thompson \& Duncan (1995).

The cooling X-ray flux from the surface of a trapped fireball is concentrated close to the surface of the star, where the magnetic scattering opacity is greatly suppressed. It has been argued that, further from the fireball surface, the escaping X-ray flux will become collimated along partly open magnetic field lines, as the result of the strong inequality in the scattering opacity of the two X-ray polarization modes in a super-strong magnetic field (Thompson \& Duncan 1995, 2001). In particular, the opacity of the E-mode scales as $\sim B^{-2}$ and grows rapidly with radius. Thus, matter suspended higher in the magnetosphere by the hyper-Eddington flux can provide a dense scattering screen that is optically thick to Thomson scattering, and through which the X-rays can escape only by pushing the matter to the side. The burst light curve therefore provides information about the connectivity of the magnetic field lines close to the fireball surface. As the fireball shrinks, it connects with a smaller (and therefore more regular) portion of the magnetosphere - which could explain the reduced number of sub-pulses toward the end of the burst.

In the self-similar magnetospheric model which we have described, the resonant scattering opacity varies too smoothly with angle to explain the narrow collimation of the X-ray flux observed in both giant flares. Even during a giant flare, the photon flux is too weak to break open the closed field lines at a radius of $\sim 50-100 \mathrm{~km}$ (the position of the electron cyclotron resonance of a $\sim 40 \mathrm{keV}$ photon). The energy density in the magnetic field exceeds that in the freely streaming X-rays by a large factor $\sim 10^{6}\left(L_{X} / 10^{42} \mathrm{erg} \mathrm{s}^{-1}\right)^{-1}(\hbar \omega / 40 \mathrm{keV})^{4 / 3}\left(B_{\text {pole }} / 10^{14} \mathrm{G}\right)^{2 / 3}$ at the resonant surface, and so the radiation pressure imparts only a negligible distortion to the field lines. Thus, we must consider whether a sudden increase in the magnetospheric current during the first $\sim 1$ second of the flare (when most of the flare energy was probably released into the magnetosphere) could force the multiple X-ray beams to be re-scattered at the electron cyclotron resonance, and thereby isotropized.

During an outburst, the current-carrying electrons feel an enormous drag force where their cyclotron energy lies in the X-ray range, independent of the sign of the current (Appendix B). In the quiescent state, the electrons move in opposite directions (both toward the neutron star and away from it) on different portions of a closed current loop. However, charged particles may be injected into the magnetosphere at a much higher rate during an SGR outburst, than they need be to support the current associated with a static twist. The quiescent charge flow is given by eq. (18), and can be converted to a kinetic 
luminosity

$$
L_{\text {matter }} \sim \frac{I(R)}{e} m_{p} c^{2}=1 \times 10^{35}\left(\frac{B_{\text {pole }}}{10^{14} \mathrm{G}}\right)^{1 / 3}\left(\frac{\hbar \omega}{\mathrm{keV}}\right)^{2 / 3}\left(\frac{R_{\mathrm{NS}}}{10 \mathrm{~km}}\right) \quad \mathrm{erg} \mathrm{s}^{-1} .
$$

(Here we have expressed the resonant radius in terms of the cyclotron photon energy.) This luminosity is comparable to the persistent X-ray luminosity (see eq. [34]), but is less than one part in a million of the bursting luminosity. In a giant flare, the radiation pressure is high enough to advect matter outward at relativistic speed from the heated neutron star surface (Appendix B; Ibrahim et al. 2001). Only a minuscule fraction of the bursting luminosity need be carried by this entrained matter, in order to supply the charges which support a static current flowing along the twisted magnetic field.

The current can be maintained by a small differential drift of the ions with respect to the electrons and photons, in the presence of a dense wind from the neutron star surface. The cyclotron energy of the ions is too small to couple them tightly to the X-rays at the same radius where the electrons are tightly coupled. This allows the X-rays to flow outward, largely unimpeded, independent of the sign of the current. However, because the electrons are tightly coupled to the photons, a multipolar pattern can be maintained in the X-ray flux only if the dense matter transported to large radius reflects the multipolar pattern of the magnetic field close to the source.

It should also be noted that the pulse profile is measured in a much higher energy range during the flare (25-100 keV for Ulysses and 40-700 for BeppoSAX) than it is in quiescence (typically $2-10 \mathrm{keV}$ ). As a result, the pulse profile is more sensitive to the presence of higher magnetic multipoles during outburst, than in quiescence.

\subsubsection{Implications of the Variable Quiescent Pulse Profile of SGR 1900+14}

The 2-10 keV pulse profile of SGR 1900+14 was complicated and multi-peaked before the 27 August 1998 giant flare (Hurley et al. 1999c). Our explanation for the change in the pulse profile following the flare (to a single sinusoidal pulse) involves a re-scattering of the X-rays at the electron-cyclotron resonance. If during the flare an additional twist was implanted in the magnetosphere, then there was at the same time an increase in the current density and the optical depth to resonant scattering.

The smooth, single pulse observed following the flare implies a simple - predominantly dipolar - geometry of the poloidal magnetic field at a distance of $\sim 50-100 \mathrm{~km}$ from the neutron star. We can then derive an upper bound to the strength of the surface quadrupole. 
Neglecting departures from axisymmetry and reflection symmetry, the poloidal field can be decomposed as

$$
B_{\theta}(R, \theta=\pi / 2)=\sum_{\ell=1}^{\infty} B_{\ell}\left(R_{\mathrm{NS}}\right)\left(\frac{R}{R_{\mathrm{NS}}}\right)^{-(\ell+2)}
$$

at the magnetic equator. Here, $B_{1}=\frac{1}{2} B_{\text {pole }}$, and higher $\ell$ represent higher multipoles. We require that the quadrupole $\ell=2$ remain weaker than the dipole $\ell=1$ at the electron cyclotron resonance of a $10 \mathrm{keV}$ photon. Expressing the resonant radius as $R_{\mathrm{res}} / R_{\mathrm{NS}}=\left(B_{1} / B_{\mathrm{QED}}\right)^{1 / 3}(\hbar \omega / \mathrm{keV})^{-1 / 3}$, one deduces

$$
\frac{B_{2}\left(R_{\mathrm{NS}}\right)}{B_{1}\left(R_{\mathrm{NS}}\right)} \lesssim 5\left[\frac{B_{1}\left(R_{\mathrm{NS}}\right)}{10^{14} \mathrm{G}}\right]^{1 / 3}\left(\frac{\hbar \omega}{10 \mathrm{keV}}\right)^{-1 / 3} .
$$

The complicated 2-10 keV pulse profile observed before the August 27 flare then has two explanations in our model. The first possibility is that, even before the flare, the X-ray flux was reprocessed by resonant scattering off electrons at $R \sim 50-100 \mathrm{~km}$, but that the current was not axisymmetric. The second possibility is that the current flowing along extended field lines was small, so that the complicated pulse profile resulted from anisotropic emission and scattering close to the source. For example, the ion cyclotron resonance lies in the X-ray range near the surface of a magnetar, where the field is probably dominated by higher multipoles (\$5.3).

These two models lead to differing conclusions about the overall strength of the magnetic field at the surface of SGR $1900+14$. Polar electrical currents will accelerate the rate of spindown with respect to a simple magnetic dipole, and reduce the surface field inferred from the measured period and period derivative (see \$6). The rapid spindown of the SGRs 1900+14 and 1806-20 compared with the AXPs, and the displacement of SGR $1900+14$ from the center of the nearest supernova remnant, suggests that their spindown is transiently accelerated (Thompson et al. 2000). Such a temporary increase in the rate of spindown could be effected through a global twist imparted to the magnetosphere.

In the absence of a global twist, SGR 1900+14 is inferred to have a polar dipole magnetic field $B_{\mathrm{MDR}} \sim 2 \times 10^{15} \mathrm{G}$, based on the rate of spindown before the giant flare (Kouveliotou et al. 1999). The presence of higher magnetic multipoles (needed to explain the complicated angular pattern of resonant ion scattering) then guarantees yet stronger surface fields, and allows multiple ion scattering to occur up to a high energy cutoff of $\sim 40\left(B_{\text {surface }} / 3 B_{\mathrm{MDR}}\right) \mathrm{keV}$.

A check of these ideas is provided by a relatively short $3.5 \mathrm{~s}$ burst emitted by SGR 1900+14 on 29 August 1998, two days after the giant flare. This burst was followed by 
a faint $\left(L_{X}<10^{37} \mathrm{erg} \mathrm{s}^{-1}\right)$ and very extended tail, which lasted more than $1000 \mathrm{~s}$ and gradually merged with the persistent emission (Ibrahim et al. 2001). The $2-20 \mathrm{keV}$ pulse profile in this tail had a similar shape, and maintained a constant relative phase, with the later persistent emission (Palmer 2001). Even at its peak, the radiative flux of the tail did not exceed that of the persistent emission $[2]$ by more than an order of magnitude. Thus, the pulse profile should be controlled by the same resonant scattering processes in the decaying tail, as it is in the later persistent emission, and the observed constant phase alignment of the pulse is expected.

\section{Implications for SGR/AXP spindown}

The measured spindown of SGR 1806-20 (Kouveliotou et al. 1998) and SGR 1900+14 (Kouveliotou et al. 1999; Woods et al. 1999c; Marsden et al. 1999) corresponds to a polar dipole field $B_{\text {pole }} \simeq 2 \times 10^{15} \mathrm{G}$. In the presence of a net twist, the external magnetic field drops off more slowly than $\sim R^{-3}$ (Fig. 2). The field strength $B\left(R_{\mathrm{lc}}\right)$ at the speed of light cylinder $R_{\mathrm{lc}}=c P / 2 \pi$ is then stronger than a pure dipole, $B_{\theta}\left(R_{\mathrm{lc}}\right) / B_{\text {pole }}=\frac{1}{2} p F(0)\left(R_{\mathrm{lc}} / R_{\mathrm{NS}}\right)^{-(2+p)}$. Since the rate of loss of rotational energy is $I \Omega \dot{\Omega} \sim B_{\theta}\left(R_{\mathrm{lc}}\right)^{2} R_{\mathrm{lc}}^{2} c$, the flaring of the field causes the spindown rate to increase. Equivalently, the real polar surface field decreases from the one inferred from a measured $\dot{P}$ and $P$ with increasing twist (decreasing radial index $p$ ). Compared directly with the magnetic dipole value, it is

$$
\frac{B_{\text {pole }}}{B_{\text {pole }}(p=1)}=\frac{1}{p F(0)}\left(\frac{c P}{2 \pi R_{\mathrm{NS}}}\right)^{p-1} .
$$

Notice that there is no direct dependence on $\dot{P}$ in this expression. An additional consequence is to reduce the braking index below the dipole value,

$$
n=\frac{\ddot{\Omega} \Omega}{(\dot{\Omega})^{2}}=2 p+1 .
$$

The ratio (44) is plotted in Fig. 6 for various spin periods. The true polar field is smaller by a factor $\simeq \frac{1}{3}$ when the outer magnetosphere has a twist $\Delta \phi_{\mathrm{N}-\mathrm{S}}=1$ radian, for spin periods in the range $3-10 \mathrm{~s}$; whereas a twist $\Delta \phi_{\mathrm{N}-\mathrm{S}}=1.5$ radian leads to a reduction of one order of magnitude in $B_{\text {pole }}$. This model has the further implication that, for a fixed

\footnotetext{
${ }^{12}$ The August 29 burst itself occurred during a period of enhanced persistent emission following the August 27 giant flare (Woods et al. 2001a); this comparison is made with the amplitude of the persistent emission recorded just before the August 29 burst.
} 
polar field, the spindown rate increases with the optical depth to resonant scattering, and hence with the hardness of the persistent X-ray spectrum. Indeed, the active SGRs 1806-20 and 1900+14 both have higher $\dot{P}$ and harder X-ray spectra than any AXP - a trend which has been further quantified by Marsden and White (2001) for the combined population of SGR and AXP sources. The quiescent SGR 0526-66 has a softer spectrum (Kulkarni et al. 2001). We predict that its spindown rate, when measured, will be intermediate between these sources and the AXPs.

It should be emphasized that if the spindown of a magnetar is persistently accelerated in this manner (so that the magnetospheric twist remains constant), then its characteristic age hardly differs from the magnetic dipole value. The spin frequency decreases as $\Omega(t) \propto t^{-2 p}$, and so the characteristic age is larger than $P / 2 \dot{P}$ by a factor $1 / p$. In the case of the AXP 1E 1841-045, the near equality between the $4 \times 10^{3}$ yr characteristic age and the age of the surrounding Kes 73 (Gotthelf et al. 1999) does not imply that its magnetic field must be close to a true dipole.

It has been previously noted (Thompson \& Blaes 1998; Harding, Contopoulos, \& Kazanas 1999; Thompson et al. 2000) that persistent seismic activity in a magnetar can also increase the rate of spindown with respect to a vacuum magnetic dipole - by triggering a fluctuating current in the magnetosphere which drives an outward flux of particles and Alfvén waves. The real polar field of SGRs 1806-20 and 1900+14 is reduced by a factor $\sim 3$ with respect to the one inferred from to the magnetic dipole formula, if the persistent seismic luminosity is equal to the observed X-ray luminosity of $\sim 10^{35} \mathrm{erg} \mathrm{s}^{-1}$. The plerionic synchrotron emission powered by a persistent particle wind of luminosity $\sim 10^{35} \mathrm{erg} \mathrm{s}^{-1}$ could, in fact, have escaped detection. Measurements of spindown in SGR $1900+14$ or SGR 1806-20 do not, however, show a direct correlation between the rate of spindown and bursting activity - with the noticeable exception of the August 27 giant flare itself (Woods et al. 1999c, 2001b). For this reason, static magnetospheric currents seem a more promising source of non-uniform spindown in the SGR and AXP sources.

Ejection of a large number of particles during a giant flare would cause a transient spindown of a soft gamma repeater. As we now show, the cumulative torque is larger if the external field is twisted, than if it is dipolar close to the star. The spin period of SGR $1900+14$ did indeed increase by $\Delta P / P=1 \times 10^{-4}$ (in comparison with the extrapolation of the previously measured spindown) within three months of the 27 August 1998 giant flare (Woods et al. 1999a). However, the torque calculated assuming a dipolar (near) field is too small by an order of magnitude if $B_{\text {pole }} \sim 10 B_{Q E D}=4.4 \times 10^{14} \mathrm{G}$, and if the particle energy $\Delta E$ and duration $\Delta t$ of the outflow are normalized to the energy and duration of 
the X-ray outburst (Thompson et al. 2000). Estimating

$$
I \frac{\Delta \Omega_{\mathrm{NS}}}{\Omega_{\mathrm{NS}}} \simeq-\frac{2}{3 c^{2}} \Delta E R_{A}^{2}
$$

where the Alfvén radius is determined by balancing the ram pressure of the particles with the magnetic tension,

$$
\frac{\Delta E / \Delta t}{4 \pi R_{A}^{2} c}=\frac{B^{2}\left(R_{A}\right)}{4 \pi}=\frac{B_{\mathrm{pole}}^{2}}{4 \pi}\left(\frac{R_{A}}{R_{\mathrm{NS}}}\right)^{-2(2+p)},
$$

one finds that the net torque is increased by a factor

$$
\frac{(\Delta P / P)_{p=0.8}}{(\Delta P / P)_{p=1}}=4\left(\frac{B_{\mathrm{NS}}}{10 B_{\mathrm{QED}}}\right)^{1 / 9}\left(\frac{\Delta E}{10^{44} \mathrm{erg}}\right)^{-1 / 18}\left(\frac{\Delta t}{400 \mathrm{~s}}\right)^{1 / 18}
$$

when $p$ is reduced from 1 to 0.8 . This brings the calculated torque close to the observed value if $B_{\text {pole }} \sim 10 B_{\mathrm{QED}}$.

\subsection{Narrow Distribution of SGR/AXP Spin Rates}

The SGR sources have spin periods measured [3] in the range 5-8 s. The distribution of spin periods for the AXP sources is remarkably similar: 6-12 s (e.g. Mereghetti 2000). While the number of sources is small enough that the detection of much shorter spin periods is not surprising, even if the sources are born spinning much more rapidly, it is surprisingly narrow given the wide range of characteristic ages $P / \dot{P}-$ from $1-3 \times 10^{3}$ yr for SGRs 1806-20 and $1900+14$ up to $4 \times 10^{5} \mathrm{yr}$ for the AXP $1 \mathrm{E} 2259+586$. It has been suggested, as a result, that the spindown of the active SGRs is transiently accelerated, and that the spindown of $1 \mathrm{E} 2259+586$ (which sits near the center of the much younger SNR CTB 109) has decayed significantly from its long term average (Thompson et al. 2000). Clearly transient acceleration is possible in this model if the AXPs are (mostly) dormant SGRs, and the external magnetic field is twisted up during periods of burst activity.

The narrow range of spin periods is suggestive of some physical process which limits the spindown rate beyond a period of $\sim 8$ seconds. We would like to point out that this period is remarkably close to the upper envelope of the distribution of spin periods in the known radio pulsar population.

\footnotetext{
${ }^{13}$ Derived from the persistent emission for SGR 1806-20 (Kouveliotou et al. 1998), from the persistent emission and 27 August 1998 giant flare for SGR 1900+14 (Hurley et al. 1999a,b; Feroci et al. 1999; Mazets et al. 1999), and from the 5 March 1979 giant flare for SGR 0526-66 (Mazets et al. 1979).
} 
The magnetospheric model which we have outlined provides a motivation for a reduction in torque above a critical spin period, where the potential drop through the magnetosphere is no longer high enough to trigger a pair cascade through emission of curvature $\gamma$-rays. In the absence of pair creation, the space-charge limited flow along open magnetic field lines will generate, in one hemisphere, a toroidal magnetic close to the magnetic axis with the opposite sign to the more global toroidal field (Fig. [). Fast reconnection of these opposing toroidal fields would have the effect of forcing a relaxation of the static twist inside the speed of light cylinder. In other words: beyond the pair death line, the intermediate regions of the corotating magnetosphere probably cannot maintain a static twist. Current will diffuse away from the region closest to the star (where the most of the current is concentrated in our self-similar solutions: eq. [18]) only on the relatively long timescale (38).

We should emphasize that we have not yet been able to demonstrate unambiguously the opposite effect: that pair creation will act to stabilize a large-scale twist. Pair creation does cause a huge multiplication in the number of charges flowing outward on open field lines, so that the pair-loaded plasma is capable of maintaining a net Goldreich-Julian charge density - even while the axial current flows in the opposite direction to the original space-charge limited flow (\$3.1). Whether the global current flow actually takes advantage of this effect is a question that we cannot presently address from first principles.

\section{Summary}

We have shown that several properties of magnetar candidates in their quiescent states become easier to understand if the neutron star's magnetic field is globally twisted. These properties are directly affected in a correlated manner by persistent magnetospheric currents. The observed X-ray pulse profile and spectrum are modified by resonant cyclotron scattering and the spindown torque is increased (for a fixed surface field strength) over the standard vacuum dipole formula.

We have idealized the magnetosphere as a twisted dipole, and constructed self-similar solutions to the force-free equation. The self-similar ansatz requires that the surface flux density and the current density (related to the twist of the field lines) have a particular dependence on the magnetic latitude which changes shape depending on the strength of the current. As the net twist between the north and south magnetic hemispheres $\Delta \phi_{\mathrm{N}-\mathrm{S}}$ increases from 0 to $\pi$, the external field continuously interpolates between a dipole and a twisted, split monopole. Since axisymmetric shear deformations of an axisymmetric star do not change the angular distribution of $B_{R}$, actual deformations of a magnetar must be 
represented by some non-linear combination of these solutions. This also suggests that the magnetosphere of a neutron star may, in practice, not be able to maintain a twist larger than $\Delta \phi_{\mathrm{N}-\mathrm{S}} \sim 1-1.5$ radians (above which the distribution of flux with polar angle begins to differ significantly from a pure dipole).

Our principal conclusions can be summarized as follows:

1. It has previously seemed difficult to make deductions about the configuration of the magnetic field in the SGR and AXP sources: one obtains evidence for the presence of higher multipoles from the light curves of the giant flares (Feroci et al. 2001; Thompson \& Duncan 2001) but not much more than that. The existence of magnetars was motivated by considerations of magnetic field amplification through dynamo activity in young, convective neutron stars: a large-scale helical dynamo is possible when the initial spin period is shorter than $\sim 3$ msec (the convective overturn time of nuclear matter from which neutrinos are escaping with a luminosity $L_{\nu} \gtrsim 10^{52} \mathrm{erg} \mathrm{s}^{-1}$ ) (Duncan \& Thompson 1992; Thompson \& Duncan 1993). Nonetheless, the connection between this theoretical dynamo model and the (now) slowly rotating SGR and AXP sources has seemed tenuous. The new results presented in this paper - which indicate the presence of strong internal toroidal fields in the SGR and AXPs - show that this theoretical picture has, at least, a degree of self-consistency.

2. A persistent current can be maintained by electrons and ions stripped from the neutron star surface, if the elemental composition is light (e.g. hydrogen, helium, or carbon). The impact of the returning ions at the cathode region of the neutron star surface, and the downward acceleration of the returning electrons at the anode surface (where the ions are accelerated upward) generates a luminosity $L_{X} \sim 10^{35}\left(B_{\text {pole }} / 10^{14} \mathrm{G}\right) \mathrm{erg} \mathrm{s}^{-1}$ in surface X-ray emission if the entire magnetosphere is twisted. This luminosity provides a lower bound to the electromagnetic output of a neutron star with an actively decaying magneic field and is, by coincidence, comparable to the passive X-ray flux powered by ambipolar diffusion in the neutron star core (TD96; Heyl \& Kulkarni 1998). The rate of surface heating would be lower by a factor $\sim \gamma_{e} m_{e} / m_{p}$ if the current were carried by relativistic $e^{ \pm}$pairs of Lorentz factor $\gamma_{e}$.

A significant rate of surface heating leads to the interesting possibility of an emission line at the surface ion cyclotron frequency. The magnetospheric charges are stopped mainly by ion collisions in magnetic field much stronger than $B_{\mathrm{QED}}$. The stopping depth is higher than in the case of a non-magnetic atmosphere, and the broad-band spectrum will, as a result, be closer to a black body than in the weak-field regime analyzed by Zel'dovich \& Shakura (1969) and Deufel, Dullemond, \& Spruit (2001).

3. A significant optical depth to resonant cyclotron scattering, $\tau_{\text {res }} \sim$ 
$(v / c)^{-1}\left(B_{\phi} / B_{\theta}\right)_{\theta=\pi / 2}$, is generated by the current carriers at the magnetic equator. This optical depth is anisotropic and approaches zero at the magnetic axis, where the equilibrium current density is $J(\theta) \propto \theta^{2}$. When the twist is large, $\left(B_{\phi} / B_{\theta}\right)_{\theta=\pi / 2}=O(1)$, photons will experience multiple resonant scattering, forming a high energy non-thermal tail. At a fixed $L_{X}$, the hardness of this tail will increase with the strength of the overall twist imparted to the magnetosphere, and so one obtains an explanation for the trend of increasing spectral hardness with overall burst activity in the combined population of SGR and AXP sources.

In our self-similar model, $\tau_{\text {res }}$ is independent of the charge and mass of the particles, as well as the resonant radius. The large difference in the charge/mass ratio of electrons and ions therefore allows a magnetar to have more than one scattering corona, localized at quite different radii: $R \sim 50-100 \mathrm{~km}$ for electrons and $\sim 10-20 \mathrm{~km}$ for ions. Ions will resonantly scatter X-rays only below the (Doppler-shifted) surface cyclotron frequency, and so the spectral tail generated by ion cyclotron scattering will be cut off at a much lower frequency than in the case of resonant electron scattering. Measurements of the persistent emission above $\sim 30 \mathrm{keV}$ can test the relative importance of the two mechanisms.

The overall similarity in the luminosities of the thermal and non-thermal components of SGR and AXP spectra has a simple interpretation in this model, but would be more difficult to understand if the non-thermal emission arose from an independent radiative process (such as synchrotron or curvature emission) in the magnetosphere. For these sources, the mechanism of multiple cyclotron scattering also has significant advantages over non-resonant Comptonization in a thin surface layer which is heated by magnetospheric charges (Zel'dovich \& Shakura 1969; Deufel et al. 2001). Aside from the relatively soft spectrum of the surface emission expected in a strong magnetic field, it will also be noted that the intrinsically brightest soft gamma repeater 0526-66 has a softer spectrum than the other three SGRs (each of which are $~ 10-30$ times less luminous; Kulkarni et al. 2001).

4. The observed pulse profile is strongly modified by resonant cyclotron scattering. Three effects enter here: the strong anisotropy in the optical depth to electron cyclotron scattering, the aspherical shape of the resonant surface, and the doppler beaming of the scattered radiation resulting from the bulk motion of the charge carriers. In addition, the flux of thermal seed photons generated by the surface impact of magnetospheric charges is strongly anisotropic even in this self-similar model. Not only is the surface current inhomogeneous, but a larger fraction of the radiative flux will be carried by the O-mode (which is beamed along the local magnetic field) than is the case in passively cooling neutron stars. This second effect is enhanced if the magnetospheric current is concentrated on extended field lines (e.g. TD96). 
5. Soft Gamma Repeater flares provide prima facie evidence for sudden variations in the magnetic field, and therefore in the electrical currents flowing outside the star (Thompson et al. 2000). We have shown that the energy available in the external field can maintain these currents for as long as $\sim 30\left(B_{\text {pole }} / 10^{14} \mathrm{G}\right)$ yr, if the current is supported by electrons and ions stripped from the neutron star surface. It is interesting to note, in this regard, that SGR 0526-66, which has been quiescent as a burst source since 1983, has a persistent X-ray spectrum which is strongly non-thermal but at the same time significantly softer than the actively bursting SGRs (Kulkarni et al. 2001). This model also provides an explanation for the simplified pulse profile observed in the persistent emission of SGR 1900+14 following the 27 August 1998 giant flare, which was maintained even after the X-ray flux returned to the baseline value observed before the flare. We obtain a valuable constraint on how the magnetic field was modified during the flare: the current flowing along extended magnetic field lines actually increased during the flare, suggesting that it was triggered by the release of sub-surface stresses.

6. Given a fixed polar magnetic field $B_{\text {pole }}$, the observed rate of spindown will grow as the external field is twisted up, and there is an increase in the fraction of the field lines which open out across the speed-of-light cylinder. Equivalently, the real surface polar field is reduced by a factor 3 for a net twist $\Delta \phi_{\mathrm{N}-\mathrm{S}}=1$ radian, in comparison with the dipole formula (Fig. 6).

7. Consideration of the stability of a twisted, force-free magnetosphere leads to the requirement that, close to the magnetic axis, the current flows in the same direction on both closed and open field lines. Such an alignment of the currents can be achieved only if the charge flow is pair-loaded on open field lines - which allows a differential drift between positive and negative charges to maintain a current opposite to $\rho_{G J} c \hat{R}$ in one hemisphere. There is, in turn, a limiting spin period beyond which the rate of spindown can no longer be accelerated with respect to an orthogonal vacuum dipole. If the pair cascade cuts off at a spin period comparable to the maximum observed in the known radio pulsar population, then one obtains an explanation for the observed narrow distribution $(P=6-12 \mathrm{~s})$ of AXP spins, and the similarly narrow distribution $(P=5-8 \mathrm{~s})$ of SGR spins. Direct evidence for such a decay in the torque is provided by the anomalous pulsar $1 \mathrm{E} 2259+586$, which has a characteristic age at least a factor of 10 larger than the age of the SNR in which it resides (Thompson et al. 2000). 


\subsection{Relation between the SGRs, AXPs, and the Soft X-ray Pulsars}

We collect, in this section, the various threads which link our model of non-potential neutron star magnetospheres to the observed behavior of the Soft Gamma Repeaters, Anomalous X-ray Pulsars, and their possible cousins, a growing group of Soft X-ray Pulsars.

\subsubsection{Polar Dipole Fields of the SGRs/AXPs and their Relation to Radio Pulsar Fields}

The polar magnetic fields of the two rapidly spinning down SGRs 1806-20 and 1900+14 (Kouveliotou et al. 1998, 1999, Woods et al. 2001b) are inferred to be $B_{\text {pole }}=1-3 \times 10^{15} \mathrm{G}$ from the standard magnetic dipole formula. These fields lie a factor of 10-30 above the strongest fields measured in the radio pulsar population, $B_{\text {pole }}=10^{14} \mathrm{G}$ (Camilo et al. 2000). The polar fields inferred analogously for the AXPs are continuous with the pulsar distribution: the source $1 \mathrm{E} 2259+586$ has a long characteristic age $P / 2 \dot{P}=2.3 \times 10^{5}$ yr and polar dipole field $1.2 \times 10^{14} \mathrm{G}$. However, most of the AXPs have nominal dipole fields 5 -10 times this value.

In our model, the actual polar magnetic fields of these sources will lie below the classical magnetic dipole value. A reduction of $\sim 3-10$ is plausible in some sources (corresponding to net twist angles of $\sim 1-1.5$ radians). The higher spindown rates measured in the SGRs, as compared with the AXPs, could simply represent a greater degree of magnetospheric twist imparted by deformations of the magnetic field that are associated with bursting activity. Indeed, the two AXPs with the fastest spindown also have the hardest persistent X-ray spectra (e.g. Table 2 of Kaspi et al. 2001). This positive correlation between spindown rate and spectra becomes even stronger when the SGRs and AXPs are lumped together (Marsden \& White 2001). Nonetheless, some distribution of surface fields is almost certainly present in the AXP and SGR populations, and it is plausible that some of the quiescent sources really do have weaker surface fields than the bursting sources.

Additional physical constraints on the dipole fields of the SGR sources come from these independent lines of argument:

i) Confinement of the relativistically hot plasma which powered the pulsating tails of the two giant flares requires magnetic fields stronger than $10^{14}\left(E / 10^{44} \mathrm{erg}\right)^{1 / 2} \mathrm{G}$ (Thompson \& Duncan 1995). This argument has been generalized to allow for the possibility that the dipole is offset from the center of the star, which would reduce the magnetic moment

\footnotetext{
${ }^{14}$ This polar field exceeds by a factor 2 the average surface field usually quoted in the radio pulsar literature.
} 
corresponding to a fixed plasma energy $E$ (Thompson \& Duncan 2001). Even including this effect, one deduces $B_{\text {pole }} \gtrsim 10^{14} \mathrm{G}$, because a compact fireball would have a very high internal temperature $T \gg 1 \mathrm{MeV}$ and would lose its energy rapidly to neutrino radiation through $e^{+}+e^{-} \rightarrow \nu+\bar{\nu}$ (instead of the observed X-ray flux).

ii) Transient spindown of SGR $1900+14, \Delta P / P=1 \times 10^{-4}$, was observed within 3 months of the 27 August 1998 giant flare (Woods et al. 1999c). A plausible mechanism for this torque involves a particle wind during the giant flare itself, combined with scattering of the X-rays by matter suspended in the magnetosphere near $R \sim 200 \mathrm{~km}$ (where the momentum flux is high enough to break open the magnetic field lines). If the magnetic field were dipolar inside this 'Alfvén' radius, then the maximum torque would be $\Delta P / P \simeq 10^{-5}\left(B_{\text {pole }} / 10 B_{Q E D}\right)$ (Thompson et al. 2000). However, if the magnetic field were twisted close to the star, then the Alfvén radius would increase. The net torque would be brought close to the observed value if $B_{\text {pole }} \gtrsim 5 \times 10^{14} \mathrm{G}$ (eq. [48]).

iii) The surface X-ray flux predicted by our self-similar model (eq. [34) is comparable to the observed luminosities of the SGR and AXP sources if $B_{\text {pole }} \sim 10^{14} \mathrm{G}$, but is excessively large if $B_{\text {pole }}$ is as large as $10^{15} \mathrm{G}$. However, it should be emphasized that the internal stresses acting on the crust of a magnetar may be more localized, and its entire magnetosphere need not be twisted. The polar field could be as strong as $\sim 10^{15} \mathrm{G}$ if the current were intermittent and flowed only over a fraction of the neutron star surface.

All of these arguments point to dipole fields stronger than $10^{14} \mathrm{G}$. A polar field several times the strongest pulsar field appears needed to explain a transient spindown $\Delta P / P=1 \times 10^{-4}$ of SGR $1900+14$ by an outflowing wind during the 27 August flare. The quiescent SGR 0526-66 has a softer spectrum (Kulkarni et al. 2001). We predict that its spindown rate, when measured, will be intermediate between these sources and the AXPs.

We re-emphasize that the actual surface fields of the SGR sources are probably much stronger than the (corrected) dipole fields: the complicated pulse profile observed during the 27 August flare provides direct evidence for the presence of higher multipoles (Feroci et al. 2001; Thompson \& Duncan 2001). In addition, only magnetic fields stronger than $\sim 4 \times 10^{15} \mathrm{G}$ will experience rapid ambipolar diffusion through the core of a magnetar (TD96; Heyl \& Kulkarni 1998).

To summarize: our model indicates that the distribution of true polar (dipole) magnetic fields of the SGRs/AXPs is significantly narrower than the classical dipole formula would suggest. The range of spindown rates is broadened by magnetospheric currents. The true polar fields of the SGRs and AXPs are continuous with the distribution of radio pulsar fields; but are probably stronger than $10^{14} \mathrm{G}$ in some sources. Radio pulsars correspond to 
those sources in which the toroidal field is either absent, or too weak to shear the crust. Furthermore if an accelerated torque in the SGR/AXP sources is associated with an active pair cascade on open field lines, then strong-B fields do not, in themselves, suppress pair creation: there must be a greater similarity in this regard between active magnetars and ordinary radio pulsars, than some calculations have suggested.

\subsubsection{Relation between the SGRs and AXPs}

The SGRs are distinguished from the AXPs by the emission of bright X-ray outbursts and, in their quiescent states, harder X-ray spectra and faster spindown. We have shown that the last two properties can be explained by a greater degree of twist in the external magnetic field. In addition, the sudden untwisting of an (internal) magnetic field provides an attractive mechanism for powering the giant flares of the SGRs. This model suggests that some AXPs may be dormant SGRs: indeed, the SGRs go through long periods of quiescence (the LMC source SGR 0526-66 has not been observed to burst since 1983; Golenetskii et al. 1987).

Even in such a unified description of the SGRs and AXPs, the question remains as to whether one type of activity typically precedes the other in a given source, or whether instead an AXP will undergo sporadic intervals of SGR activity which are separated by periods of silence as a burst source. In fact, some AXPs may never manifest SGR behavior. It has been suggested by Gaensler et al. (2001) that the AXPs are characteristically younger than the SGRs, because 3 of the 6 AXP sources are situated very close to the centers of supernova remnants. On the other hand, the other 3 AXPs do not have obvious SNR counterparts, and one soft gamma repeater (SGR 1806-20) sits close to the center of the radio nebula G10.0-0.3 (Kulkarni et al. 1994). Circumstantial evidence for high proper motions in two SGRs comes from the projected position of SGR 0526-66 near the edge of the LMC remnant N49 (Cline 1982), and the position of SGR 1900+14 just outside SNR G42.8+0.6 (Hurley et al. 1999b). A systematic difference in proper motions between the AXPs and SGRs, if real, is an important clue to the conditions which give rise to these sources, but our model does not offer any unambiguous suggestion for what that difference may be.

The short spindown ages of SGRs $1900+14$ and $1806-20(P / \dot{P}<3000$ yrs: Kouveliotou et al. 1998, 1999; Woods et al. 2001c) seem to provide evidence, at first sight, that these sources are younger than most of the AXPs. This impression could, however, be an artifact of a sufficiently strong twisting of the external field. If the sources are in fact older (as their positions with respect to the nearest SNR would suggest) then their spindown must 
be persistently but transiently accelerated with respect to the AXP population (Thompson et al. 2000). Combining the two populations, any AXP must spend at most $\sim 25 \%$ of its $\sim 10^{4}-10^{5}$ yr lifetime as a bright X-ray source in an SGR mode (Thompson et al. 2000). However, the length of any given interval of SGR activity is poorly constrained at present: it must be at least $10-20$ yrs, but could easily be much longer than that.

We conclude that no unambiguous sequence of SGR and AXP activity is discernable from the data, in part because the spindown torques of the bursting sources appear to be accelerated transiently, and also because a wide range of proper motions may exist in the combined population of SGRs and AXPs. A plausible scenario is one in which a portion of the AXP population undergoes intermittent periods of bursting activity. It should be kept in mind that SGR activity may be concentrated during a particular range of ages, when the crust of the star is colder and more brittle. Because the spindown torque may be increased substantially by global magnetospheric currents, the true polar fields of the actively bursting SGRs need not lie at the extreme high end of the magnetar population.

This model is testable by long-term monitoring of the spin of an SGR source, after it ends a period of bursting activity. Thus, continuous (phase-connected) monitoring of these sources is crucial to the unraveling of the relation between the SGR and AXP phenomena.

\subsection{Connection between the SGRs and AXPs and nearby Soft X-ray Pulsars}

The nearby soft X-ray pulsars RX J0420.0-5022, RX J0720.4-3125, and RBS 1223 have spin periods $(P=22.7,8.37$ and 5.2 s; Neuhäuser \& Trümper 1999) remarkably close to the SGRs and AXPs. It has been noted (Heyl \& Hernquist 1998; Kulkarni \& van Kerkwijk 1998) that these sources may be aged magnetars. They are much fainter X-ray sources than the SGRs and AXPs and could, at earlier times, have been observable either as radio pulsars or as SGRs/AXPs. (The microphysical heating mechanism most plausibly is a combination of ohmic decay and Hall deformations in the neutron star crust, because ambipolar diffusion of a magnetic field through the core should be largely frozen when the surface temperature is as low as $\sim 60-100 \mathrm{eV}$.) If these objects are evolved from the SGR/AXP population, then they provide further evidence for a decay of the torque beyond a characteristic spin period. The recent possible detection of rapid spindown in the source RBS 1223 implies a surprisingly short characteristic age of $\sim 10^{4}$ yrs (Hambaryan et al. 2001), which is not consistent with this simple scenario. 


\subsection{Transient Effects}

This model also provides a basis for interpreting time-dependent effects observed in the SGR and AXP sources, including variations in flux and pulse profile (Iwasawa, Koyama, \& Halpern 1992; Woods et al. 2001a), and variations in torque (Paul et al. 2000; Kaspi et al. 2001; Woods et al. 2001b). Indeed, these transient effects probably provide the strongest constraints on the physical processes operating in the magnetospheres of the SGR and AXPs.

We are optimistic that progress in understanding the electrodynamics of neutron stars with actively decaying magnetic fields - magnetars - will occur more rapidly than has been the case with radio pulsars - for the simple reason that the observations place many more direct constraints on theoretical models.

We thank Phil Arras, Vicky Kaspi, Chryssa Kouveliotou, and Peter Woods for comments and stimulating discussions. CT acknowledges the support of the NSERC of Canada, and the Alfred P. Sloan Foundation. ML acknowledges the support of a CITA National Fellowship. CT and ML also thank the Institute for Theoretical Physics at the University of California at Santa Barbara (NSF grant PHY99-0749) for its support during the workshop on 'Spin, Magnetism and Rotation in Young Neutron Stars', when part of this work was done.

\section{A. Polarization Mode Exchange.}

In this appendix we show that even near the center of the cyclotron resonance, the dielectric properties of the magnetosphere are dominated by vacuum polarization. The plasma contribution to the refractive index is

$$
|n-1|_{\text {plasma }}=\frac{2 \pi Z e n_{Z} c}{B \Delta \omega}
$$

at a frequency $\omega=Z e B / M c \pm \Delta \omega$. (Here $Z e, M$ and $n_{Z}$ are the charge, mass and density of the resonant particles, which could either be electrons or ions.) Estimating $Z e n_{Z} \sim B / 4 \pi R$ through Ampère's equation, this gives

$$
|n-1|_{\text {plasma }} \sim \frac{1}{4 \pi}\left(\frac{\lambda}{R}\right)\left(\frac{\Delta \omega}{\omega}\right)^{-1},
$$


independent of $Z$ and $M$, but depending on the wavelength $\lambda=2 \pi c / \omega$ of the X-ray photon. By contrast, the vacuum contribution to the index of refraction is

$$
|n-1|_{\text {vacuum }}=K \alpha_{\mathrm{em}}^{2}\left(\frac{B}{B_{\mathrm{QED}}}\right)^{2} \sin ^{2} \theta=K \alpha_{\mathrm{em}}^{2}\left(\frac{\hbar \omega}{m_{e} c^{2}}\right)^{2} \sin ^{2} \theta,
$$

which we have evaluated at the electron cyclotron resonance $\hbar \omega=\left(B / B_{\mathrm{QED}}\right) m_{e} c^{2}$. In this expression $\alpha_{\mathrm{em}}=\frac{1}{137}$, and the constant $K=\frac{7}{90}$ for the O-mode and $\frac{2}{45}$ for the E-mode. The characteristic width of the resonance is determined by the thermal motion, $\Delta \omega / \omega \sim\left(k T / m_{e} c^{2}\right)^{1 / 2}=0.04(k T / \mathrm{keV})^{1 / 2}$. We conclude that the vacuum contribution is the larger by a factor $\sim 10^{4}(\hbar \omega / \mathrm{keV})^{3}(\Delta \omega / \omega)$ at the electron cyclotron resonance (radius $R \sim 100 \mathrm{~km}$ for magnetar-strength fields).

The good photon polarization states are linear in this regime, and both are absorbed and emitted at the cyclotron resonance (which interacts with an elliptically polarized mode). The net result is that cyclotron scattering, at both ion and electron resonances, will reduce the polarization of X-rays escaping the star in a direction almost parallel to the local magnetic field, and will induce a net linear polarization of X-rays escaping across closed magnetic field lines (with the polarization vector lying perpendicular to $\mathbf{B}$ ). Thus, measurements of the emergent X-ray polarization (Heyl \& Shaviv 2000) will provide a direct probe of the current flowing through the atmosphere of a magnetar.

\section{B. Resonant Cyclotron Force Vs. Gravity}

The radiation field is anisotropic everywhere in the magnetosphere, and a particle at rest will feel a force from resonant cyclotron scattering. We now show that if the surface $\mathrm{X}$-ray flux is powered self-consistently by the impact of magnetospheric charges (\$5.1), then the radiative force acting on electrons is typically large compared with gravity. The conclusion is slightly more complicated for ions: in the quiescent state of an SGR or AXP source, the radiative force acting on them is typically small compared with gravity at the surface of the star. The radiative force remains weaker than gravity at greater distances if the spectral intensity $L_{\omega}$ increases with frequency below $\hbar \omega \sim 1 \mathrm{keV}\left(L_{\omega} \sim \omega^{\alpha}\right.$ with $\left.\alpha>0\right)$.

\section{B.1. Radiative Force}

The radiative force is easily estimated when the magnetic field is purely radial, and the radiation field is axially symmetric about $\mathbf{B}$. Because the re-emitted photon carries 
vanishing average momentum, this force is

$$
F_{\mathrm{rad}}=\int d \omega \int_{0}^{1} 2 \pi d(\cos \theta)\left[\frac{d L_{\omega}}{d \Omega} \cos \theta\right] \frac{\sigma_{\mathrm{res}}(\omega, \cos \theta)}{4 \pi R^{2} c}
$$

(we neglect the effect of the recoil; cf. Sincell \& Krolik 1992). Substituting $\sigma_{\text {res }}(\omega, \cos \theta)=\left(Z^{2} \pi^{2} e^{2} / M c\right)\left(1+\cos ^{2} \theta\right) \delta\left(\omega-\omega_{c}\right)$ near the cyclotron frequency $\omega_{c}=Z e B / M c$, one finds (see Mitrofanov \& Pavlov 1982 for the case of electron scattering)

$$
F_{\mathrm{rad}}=K_{\mathrm{rad}} \frac{Z \pi^{2} e^{2}}{e B} \frac{\left(\omega L_{\omega}\right)_{\omega_{c}}}{4 \pi R^{2} c} .
$$

(The numerical coefficient $K_{\text {rad }}=2$ in the case of a purely radial photon field; whereas $K_{\text {rad }}=\frac{3}{4}$ near the surface of a black body.) Comparing with the gravitational force $F_{\text {grav }}=G M M_{\mathrm{NS}} / R^{2}$ on the scattering charge, and substituting eq. (34) for $L_{X}=\int L_{\omega} d \omega$, one finds

$$
\frac{F_{\text {rad }}}{F_{\text {grav }}}=\frac{\pi K_{F} Z p F(\pi / 2)}{8(2+p)}\left(\frac{M}{m_{p}}\right)^{-1}\left(\frac{B}{B_{\text {pole }}}\right)^{-1}\left[\frac{\left(\omega L_{\omega}\right) \omega_{c}}{L_{X}}\right]\left(\frac{B_{\phi}}{B_{\theta}}\right)_{\theta=\pi / 2} .
$$

The numerical coefficient in front is $O\left(10^{-1}\right)$, and for electrons one deduces

$$
\frac{F_{\text {rad }}}{F_{\text {grav }}} \sim 10^{2}\left(\frac{R_{\text {res }}}{R_{\mathrm{NS}}}\right)^{3}\left[\frac{\left(\omega L_{\omega}\right)_{\omega_{c}}}{L_{X}}\right]\left(\frac{B_{\phi}}{B_{\theta}}\right)_{\theta=\pi / 2} \gg 1 \quad \text { (electrons). }
$$

(If the twist is very small, then internal heating can easily power a large enough surface $\mathrm{X}$-ray flux to enforce the same inequality.)

By contrast, ions will interact resonantly with X-rays at the surface of the star in the presence of $\sim 10^{14}-10^{15} \mathrm{G}$ magnetic fields, and one deduces

$$
\frac{F_{\text {rad }}}{F_{\text {grav }}} \sim 0.1\left(\frac{Z}{A}\right)\left[\frac{\left(\omega L_{\omega}\right) \omega_{c}}{L_{X}}\right]\left(\frac{B_{\phi}}{B_{\theta}}\right)_{\theta=\pi / 2} \lesssim 1 \quad \text { (ions). }
$$

(Atoms of large atomic number $Z$ will become bound in molecular chains in magnetarstrength fields: Lai \& Salpeter 1997; Thompson et al. 2000.)

The radiation force on the ions can overcome gravity at the surface of the star only if the luminosity $\left(\omega L_{\omega}\right)_{\omega_{c, p}}$ of X-rays at the surface cyclotron frequency is much higher than eq. (34). One possible example of such a situation is the burst from SGR $1900+14$ on 29 August 1998, which showed an extended faint tail of X-ray emission with a very hard spectrum $(k T \gtrsim 4 \mathrm{keV})$. That tail may represent transient cooling of a relatively small surface hotspot following the dissipation of a very hot magnetospheric plasma (Ibrahim et al. 2001). The radiative force at the line would also be increased in the presence of surface 
heating by magnetospheric charges; but the mildly relativistic upward motion of the ions would quickly shift them out of resonance with the surface cyclotron frequency. Finally, it has been suggested that in some magnetar candidates, the steep high energy spectral tail extends to frequencies well below $\hbar \omega \sim 1 \mathrm{keV}$ (Kulkarni et al. 2001). In that case, it would be possible for the radiative force on the ions to exceed gravity beyond a certain distance from the star.

\section{B.2. Draining Suspended Material through Persistent Currents}

Notice that in the case of a neutral ion-electron plasma, the radiative force on the electrons indicated by eq. ( $(\mathbb{B} 4)$ could exceed gravity by more than a factor $\sim(A / Z)\left(m_{p} / m_{e}\right)$, thereby allowing plasma which is blown into the magnetosphere during an X-ray flare to be supported in the magnetosphere against gravity as the X-ray flux returns to the baseline value. However, the mass of plasma which can be so supported (in e.g. a thin disk near the magnetic equator: Zheleznyakov \& Serber 1994) is small enough that it would quickly be drained by even a relatively weak magnetospheric current. Balancing the radiation pressure normal to the 'disk' against the normal component of gravity at a scale height $h$ above the magnetic equator,

$$
\frac{\left(\omega L_{\omega}\right)_{\omega_{c}}}{4 \pi R^{2} c}\left(\frac{h}{R}\right) \lesssim \Sigma \frac{G M_{\mathrm{NS}}}{R^{2}}\left(\frac{h}{R}\right),
$$

gives the maximum surface density which can be supported against gravity by resonant scattering,

$$
\Sigma \lesssim \frac{\left(\omega L_{\omega}\right)_{\omega_{c}}}{4 \pi G M_{\mathrm{NS}} c} .
$$

(This estimate requires a large optical depth to resonant scattering $\tau_{\text {res }} \sim F_{\text {rad }} / F_{\text {grav }}$, which can indeed be maintained when the radiative force $F_{\text {rad }}$ acting on the electrons is stronger than gravity.)

The flux of charges across the magnetic equator at radius $R$ is $J / e \sim$ $(c / 4 \pi)\left(2 B_{\theta} / R\right)\left(B_{\phi} / B_{\theta}\right)_{\theta=\pi / 2}$. If a source of charges is available in the magnetosphere, it is energetically favorable for the current to tap it (instead of being drawn from charges lifted off the neutron star surface). Thus, one can expect the 'disk' to be drained by the current, in a very short time:

$$
\begin{aligned}
t_{\mathrm{drain}}=\frac{2 \Sigma / m_{p}}{J / e} & \sim \frac{R_{\mathrm{NS}}}{c}\left(\frac{\left(\omega L_{\omega}\right)_{\omega_{c}}}{G M_{\mathrm{NS}} B_{\mathrm{pole}} m_{p} c / e}\right)\left(\frac{R}{R_{\mathrm{NS}}}\right)^{4}\left(\frac{B_{\phi}}{B_{\theta}}\right)_{\theta=\pi / 2}^{-1} \\
& \sim 0.03\left[\frac{\left(\omega L_{\omega} \omega_{c}\right.}{L_{X}}\right]\left(\frac{R}{10 R_{\mathrm{NS}}}\right)^{4} \mathrm{s.}
\end{aligned}
$$


Notice that this expression has no explicit dependence on the magnitude of the twist, if the surface X-ray flux is powered self-consistently by the impact of magnetospheric charges.

Matter can also be suspended by the centrifugal force beyond the corotation radius,

$$
R_{\mathrm{co}}=\left(G M_{\mathrm{NS}}\right)^{1 / 3} \Omega_{\mathrm{NS}}^{-2 / 3}
$$

even if the radiative force is weaker than gravity. The maximum column density which can be so suspended can be estimated by balancing the ram pressure of material corotating with the star, with the local magnetic tension (Thompson 2000; Ibrahim et al. 2001),

$$
\frac{\Sigma}{2 h}\left(R_{\mathrm{co}} \Omega_{\mathrm{NS}}\right)^{2} \simeq \frac{B_{\theta}^{2}\left(R_{\mathrm{co}}\right)}{8 \pi}
$$

Here $h$ is the scale height of the plasma above the magnetic equator. Substituting eq. (Bי), and re-expressing $\Sigma$ in terms of a Thomson optical depth, we obtain

$$
\tau_{T} \simeq \frac{\Sigma \sigma_{T}}{m_{p}}=2\left(\frac{B_{\text {pole }}}{10^{15} \mathrm{G}}\right)^{2}\left(\frac{h / R_{\mathrm{co}}}{10^{-2}}\right)\left(\frac{P}{6 \mathrm{~s}}\right)^{-8 / 3}
$$

(for $M_{\mathrm{NS}}=1.4 M_{\odot}$ and $R_{\mathrm{NS}}=10 \mathrm{~km}$ ). The scale height can be estimated as

$$
\frac{h\left(R_{\mathrm{co}}\right)}{R_{\mathrm{co}}} \sim\left(\frac{k T}{m_{p} g\left(R_{\mathrm{co}}\right) R_{\mathrm{co}}}\right)^{1 / 2}
$$

where the temperature of the plasma is reduced from the surface value by the factor $T / T_{S} \sim\left(h / R_{\mathrm{co}}\right)^{1 / 4}\left(R_{\mathrm{co}} / R_{\mathrm{NS}}\right)^{-1 / 2}$. This gives

$$
\frac{h\left(R_{\mathrm{co}}\right)}{R_{\mathrm{co}}} \sim 0.004\left(\frac{k T_{S}}{0.5 \mathrm{keV}}\right)^{4 / 7}\left(\frac{P}{6 \mathrm{~s}}\right)^{4 / 21} .
$$

The normalization $B_{\text {pole }} \sim 10^{15} \mathrm{G}$ of the surface dipole field in eq. (B11) is appropriate to the actively bursting SGRs 1806-20 and 1900+14, if they have purely dipolar magnetic fields (Kouveliotou et al. 1998, 1999). However, we have seen (in $\S 6$ ) that the real value of $B_{\text {pole }}$ is reduced if the magnetic field decreases with radius more slowly than a dipole, $\mathbf{B} \sim R^{-(2+p)}$ with $p<1$. This also has the effect of increasing the mass which can be contained by the magnetic tension against the centrifugal force. For a fixed $P$ and $\dot{P}$, the net effect is to re-scale $B_{\text {pole }}$ from the magnetic dipole value by the factor

$$
B_{\text {pole }} \rightarrow\left(\frac{\Omega R_{\mathrm{co}}}{c}\right)^{1-p} B_{\text {pole }}(\mathrm{MDR})<B_{\text {pole }}(\mathrm{MDR})
$$

This factor is $0.55(P / 6 \mathrm{~s})^{-0.05}$ in the case $p=0.85$. Combining it with eq. (B13) in eq. (B11), we conclude that the suspended matter can maintain a modest optical depth to Thomson scattering, $\tau_{T}>1$, when $B_{\text {pole }}(\mathrm{MDR}) \gtrsim 2 \times 10^{15} \mathrm{G}$. 
Finally, let us write down the time for a persistent current to drain this suspended material. It is

$$
t_{\text {drain }}=4\left(\frac{B_{\text {pole }}}{10^{15} \mathrm{G}}\right)\left(\frac{h / R_{\mathrm{co}}}{10^{-2}}\right)\left(\frac{B_{\phi}}{B_{\theta}}\right)_{\theta=\pi / 2}^{-1} \quad \mathrm{yr},
$$

assuming $M_{\mathrm{NS}}=1.4 M_{\odot}$ and $R_{\mathrm{NS}}=10 \mathrm{~km}$. Substituting once again eqs. (B13) and (B14), one infers a drainage time $t_{\text {drain }} \sim 1\left(B_{\text {pole }} / 10^{15} \mathrm{G}\right)\left(B_{\phi} / B_{\theta}\right)_{\theta=\pi / 2}^{-1}$ yr (quoted here for $p=0.85)$. Of course, the density of suspended material may be high enough for it to

spin outward from the star through the action of the centrifugal force, and settle into a rotationally supported disk (Thompson 2000).

\section{REFERENCES}

Banas, K.R., Hughes, J.P., Bronfman, L., \& Nyman, L.A. 1997, ApJ, 480, 607

Basko \& Sunyaev 1975, A\&A, 42, 311

Blandford, R.D. \& Payne, D.G. 1981, MNRAS, 194, 1033

Camilo, F., Kaspi, V.M., Lyne, A.G., Manchester, R.N., Bell, J.F., D’Amico, N., McKay, N.P.F., \& Crawford, F. 2000, ApJ, 541, 367

Canuto, V., Lodenquai, J., \& Ruderman, M. 1971, Phys. Rev. D3, 2303

Cline, T.L. 1982, in Gamma-Ray Transients and Related Astrophysical Phenomena, ed. R.E. Lingenfelter, H.S. Hudson \& D.M. Worrall (New York: AIP), p. 17

Deufel, B., Dullemond, C.P., \& Spruit H.C. 2001, A\&A, in press (astro-ph/0108438)

Duncan, R.C. \& Thompson, C., 1992, ApJ, 392, L9 (DT92)

Feroci, M. et al. 1999, ApJ, 515, L9

Feroci, M., Hurley, K., Duncan, R.C., \& Thompson, C., ApJ, 549, 1021

Frail, D.A. 1998, in The Many Faces of Neutron Stars, ed. R. Bucchieri, J. van Paradijs, \& M.A. Alpar (Dordrecht: Kluwer), p. 179

Frail, D.A., Kulkarni, S.R., \& Bloom, J.S. 1998, Nature, 398, 127

Fuchs Y., Mirabel, F., Chaty, S., Claret, A., Cesarsky, C.J., \& Cesarsky, D.A. 1999, A\&A, 350,891 
Gaensler, B.M., Slane, P.O., Gotthelf, E.V., \& Vasisht G. 2001, ApJ, 559, 963

Gavriil, F.P. \& Kaspi, V.M. 2001, ApJ, submitted astro-ph/0107422)

Goldreich, P. \& Julian, W.H. 1969, ApJ, 157, 869

Golenetskii, S.V., Aptekar, R.L., Guryan, Y.A., Ilinskii, V.N., \& Mazets, E.P. 1987, Sov. Astron. Lett., 13, 166

Gotthelf E.V., Vasisht, G. \& Dotani, T. 1999, ApJ, 522, L49

Haberl, F., Motch, C., Buckley, D.A.H., Zickgraf, F.-J., \& Pietsch, W. 1997, A\&A, 326, 662

Hambaryan, V., Hasinger, G., Schwope, A.D., \& Schulz, N.S. 2001, A\&A, in press (astro-ph/0110365)

Harding, A.K., Contopoulos, I., \& Kazanas, D. 1999, ApJ, 525, L125

Herold, H., Ruder, H., \& Wunner, G. 1982, A\&A, 115, 90

Heyl, J.S. \& Hernquist, L. 1997, ApJ, 489, L67

Heyl, J.S. \& Hernquist, L. 1998, MNRAS, 297, L69

Heyl, J.S. \& Kulkarni, S.R. 1998, ApJ, 506, L61

Heyl, J.S. \& Shaviv, N.J. 2000, MNRAS, 311, 555

Ho, W.C.G. \& Lai, D. 2001, MNRAS, 327, 1081

Hulleman, F., van Kerkwijk, M.H., \& Kulkarni, S.R. 2000, Nature, 408, 689

Hulleman, F., Tennant, A.F., van Kerkwijk, M.H., Kulkarni, S.R., Kouveliotou, C., \& Patel, S.K. 2001, ApJ, in press (astro-ph/0110172)

Hurley, K. 2000, in Proceedings of the Fifth Compton Symposium, ed. M.L. McConnell \& J.M. Ryan (New York: AIP), p. 515

Hurley, K., Cline, T., Mazets, E., Barthelmy, S., Butterworth, P., Marshall, F., Palmer, D., Aptekar, R., Golenetskii, S., Il'Inskii, V., Frederiks, D., McTiernan, J., Gold, R., \& Trombka, J. 1999a, Nature, 397, 41

Hurley, K., Kouveliotou, C., Woods, P., Cline, T., Butterworth, P., Mazets, E., Golenetskii, S., \& Frederics, D., 1999b, ApJ, 510, L107 
Hurley, K., Li, P., Kouveliotou, C., Murakami, T., Ando, M., Strohmayer, T., van Paradijs, J., Vrba, F., Luginbuhl, C., Yoshida, A., \& Smith, I. 1999c, ApJ, 510, L111

Hurley, K., Kouveliotou, C., Cline, T., Mazets, E., Golenetskii, S., Frederiks, D.D., \& van Paradijs, J. 1999d, ApJ, 523, L37

Ibrahim, A.I., Strohmayer, T.E., Woods, P.M., Kouveliotou, C., Thompson, C., Duncan, R.C., Dieters, S., van Paradijs, J., \& Finger, M. 2001, ApJ, in press (astro-ph/0007043)

Iwasawa, K., Koyama, K., \& Halpern, J.P. 1992, PASJ, 44, 9

Kaplan, D.L., Kulkarni, S.R., van Kerkwijk, M.H., Rothschild, R.E., Lingenfelter, R.L., Marsden, D., Danner, R., \& Murakami, T. 2001a, ApJ, 556, 399

Kaplan, D.L., Kulkarni, S.R., Frail D.A., \& van Kerkwijk, M.H. 2001b, ApJ, submitted (astro-ph/0107519)

Kaplan, D.L., Fox, D.W., Kulkarni, S.R., Gotthelf, E.V., Vasisht, G., \& Frail D.A. 2001c, ApJ, submitted (astro-ph/0108195)

Kaspi, V.M., Chakrabarty, D., \& Steinberger, J. 1999, ApJ525, L33

Kaspi, V.M., Gavriil, F.P., Chakrabarty, D., Lackey, J.R., \& Muno, M.P. ApJ, 558, 253

Kouveliotou, C., et al. 1998, Nature, 393, 235

Kouveliotou, C., et al. 1999, ApJ, 510, L115

Kulkarni, S.R. \& Frail, D.A. 1993, Nature, 365, 33

Kulkarni, S.R., Frail, D.A., Kassim, N.E., Murakami, T., \& Vasisht, G. 1994, Nature, 368, 129

Kulkarni, S.R. \& van Kerkwijk, M.H. 1998, A\&A, 507, L49

Kulkarni, S.R., Kaplan, D.L., Marshall, H.L., Frail, D.A., Murakami, T., \& Yonetoku, D. 2001, preprint

Lai, D. \& Ho, W.C.G. 2001, preprint (astro-ph/0108127)

Lai, D. \& Salpeter, E.E. 1997, ApJ, 491, 270

Lynden-Bell, D. \& Boily, C. 1994, MNRAS, 267, 146 
Lyutikov, M. \& Thompson C. 2001, in progress

Marsden, D., Rothschild, R.E., \& Lingenfelter, R. 1999, ApJ, 520, L107

Marsden, D. \& White, N.E. 2001, ApJ, 551, L155

Mazets, E.P., et al., 1979, Nature, 282, 587

Mazets, E.P., et al., 1999, preprint (astro-ph/9905196 v2)

Mereghetti, S. 2000, in 'The Neutron Star - Black Hole Connection', ed. V. Connaughton, C. Kouveliotou, J. van Paradijs, \& J. Ventura (Dordrecht: Reidel), in press (astro-ph/9911252)

Michel, F.C. 1991, 'Theory of Neutron Star Magnetospheres', (U. Chicago Press)

Mestel, L. 1993, in Pulsars as Physics Laboratories, ed. R.D. Blandford, A. Hewish, A.G. Lyne, \& L. Mestel (Oxford), p. 93

Mestel, L. \& Pryce, M.H.L. 1992, MNRAS, 254, 355

Mitrofanov, I.G. \& Pavlov, G.G. 1982, MNRAS, 200, 1033

Murakami, T., Tanaka, Y., Kulkarni, S. R., Ogasaka, Y., Sonobe, T., Ogawara, Y., Aoki, T., \& Yoshida, A. 1994, Nature, 368, 127

Murakami, T. et al. 1999, ApJ, 510, L122

Nelson, R.W., Salpeter, E.E., \& Wasserman, I. 1993, ApJ, 418, 874

Nelson, R.W., Wang, J.C.L., Salpeter, E.E., \& Wasserman, I. 1995, ApJ, 438, L99

Neuhaüser, R. \& Trümper, J.E. 1999, A\&A, 343, 151

Oosterbroek, T., Parmar, A.N., Mereghetti, S., \& Israel, G.L. 1998, A\&A, 334, 925

Özel, F. 2001, ApJ, submitted (astro-ph/0103227)

Pacini, F. 1967, Nature, 216, 567

Paczyǹski, B., 1992, Acta Astron., 42, 145

Palmer, D.M. 2001, in 'Soft Gamma Repeaters: The Rome 2001 Mini-Workshop', ed. M. Feroci, S. Mereghetti, \& L. Stella, in press (astro-ph/0103404

Paul, B., Kawasaki, M., Dotani, T., \& Nagase, F. 2000, ApJ, 537, 319 
Pivovaroff, M.J., Kaspi, V.M., \& Camilo, F. 2000, ApJ, 535, 379

Psaltis, D., Özel, F., \& DeDeo, S. 2000, ApJ, 544, 390

Rajagopal, M. \& Romani, R.W. 1997, ApJ, 491, 296

Reisenegger, A. \& Goldreich, P. 1992, ApJ, 395, 250

Ruderman, M.A. \& Sutherland, P.G. 1975, ApJ, 195, 51

Schaeffer, R., Reeves, H., \& Orland, H. 1982, ApJ, 254, 688

Scharlemann, E.T., Arons, J., \& Fawley, W.M. 1978, ApJ, 222, 297

Sincell, M.W. \& Krolik, J.H. 1992, ApJ, 395, 553

Thompson, C. 2000, in The Neutron Star-Black Hole Connection, ed. V. Connaughton, C. Kouveliotou, J. van Paradijs, \& J. Ventura (Dordrecht: Reidel), in press (astro-ph/0010016)

Thompson, C. 2001, in Soft Gamma Repeaters: The Rome 2000 Mini-Workshop, ed. M. Feroci, S. Mereghetti, \& L. Stella, in press (astro-ph/0110679)

Thompson, C. \& Blaes, O. 1998, Phys. Rev. D, 57, 3219

Thompson, C. \& Duncan, R.C. 1993, ApJ, 408, 194 (TD93)

Thompson, C. \& Duncan, R.C., 1995, MNRAS, 275, 255 (TD95)

Thompson, C. \& Duncan, R.C. 1996, ApJ, 473, 322 (TD96)

Thompson, C., Duncan, R.C., Woods, P.M., Kouveliotou, C., Finger, M.H., \& van Paradijs, J. 2000, ApJ, 543, 340

Thompson, C. \& Duncan, R.C. 2001, ApJ, 561, 000 (astro-ph/0110675)

Thompson, C. \& Murray, N.W. 2001, ApJ, 560, 000 (astro-ph/0105425)

Treves, A., Popov, S.B., Colpi, M., Prokhorov, M.E., \& Turolla R. 2000, in X-ray astronomy 2000, ed. R. Giacconi, L. Stella, \& S. Serio (San Francisco: ASP), in press

van Kerkwijk, M.H., Kulkarni, S.R., Matthews, K., \& Neugebauer, G. 1995, ApJ, 444, L33

Vrba, F.J., Henden, A.A., Luginbuhl, C.B., Guetter, H.H., Hartmann, D.H., \& Klose, S. 2000, ApJ, 533, L17 
Woltjer, L. 1958, ApJ, 128, 384

Woods, P., Kouveliotou, C., van Paradijs, J., Finger, M.H. \& Thompson, C. 1999a, ApJ, 518, L103

Woods, P., Kouveliotou, C., van Paradijs, J., Hurley, K., Kippen, R., Finger, M.H., Briggs, M., Dieters, S., \& Fishman, G. 1999b, ApJ, 519, L139

Woods, P.M., Kouveliotou, C., van Paradijs, J., Finger, M.H., Thompson, C., Duncan, R.C., Hurley, K., Strohmayer, T., Swank, J., \& Murakami, T. 1999c, ApJ, 524, L55

Woods, P.M., Kouveliotou, C., Finger, M.H., Gögüs, E., Scott, D.M., Dieters, S., Thompson, C., Duncan, R. C., Hurley, K., Strohmayer, T., Swank, J., \& Murakami, T. 2000, ApJ, 535, L55

Woods, P.M., Kouveliotou, C., Gogus, E., Finger, M.H., Swank, J., Smith, D.A., Hurley, K., \& Thompson, C. 2001a, ApJ, 552, 748

Woods, P.M., Kouveliotou, C., Gougus, E., Finger, M.H., Swank, J., Markwardt, C.B., Hurley, K., \& van der Klis, M. 2001b, ApJ, submitted (astro-ph/0109361)

Zane, S., Turolla, R., \& Treves, A. 2000, ApJ, 537, 387

Zel'dovich, Y.B. \& Shakura, N.I. 1969, Soviet Astron.-AJ, 13, 175

Zheleznyakov, V.V. \& Serber, A.V. 1994, Space Sci. Rev., 68,

This preprint was prepared with the AAS LATEX macros v4.0. 


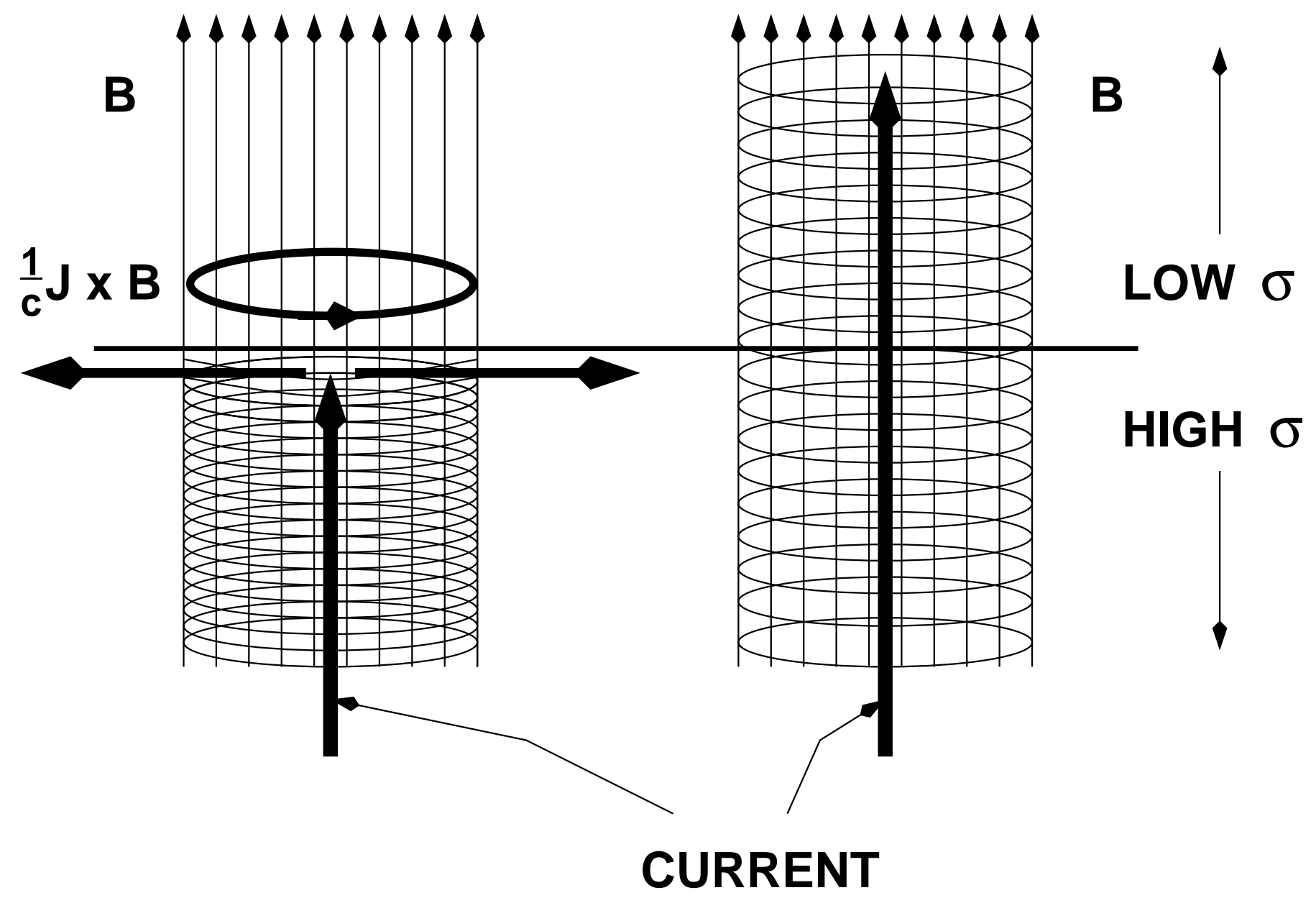


Fig. 1.- A twisted magnetic field is anchored in the highly conducting interior of a degenerate star. The twist is initially confined to the interior of the star, so that the current closes at the surface by flowing across the magnetic field. The resulting $\frac{1}{c} \mathbf{J} \times \mathbf{B}$ force causes the liquid near the surface to rotate, so as to distribute the twist more uniformly along the magnetic field lines. The net effect is to force the current to flow out of the star, into its 'magnetosphere'. In the case of a magnetar, this process may be partly stabilized by the rigidity of the crust, so that the external field twists up intermittently (giving rise to SGR flares). 


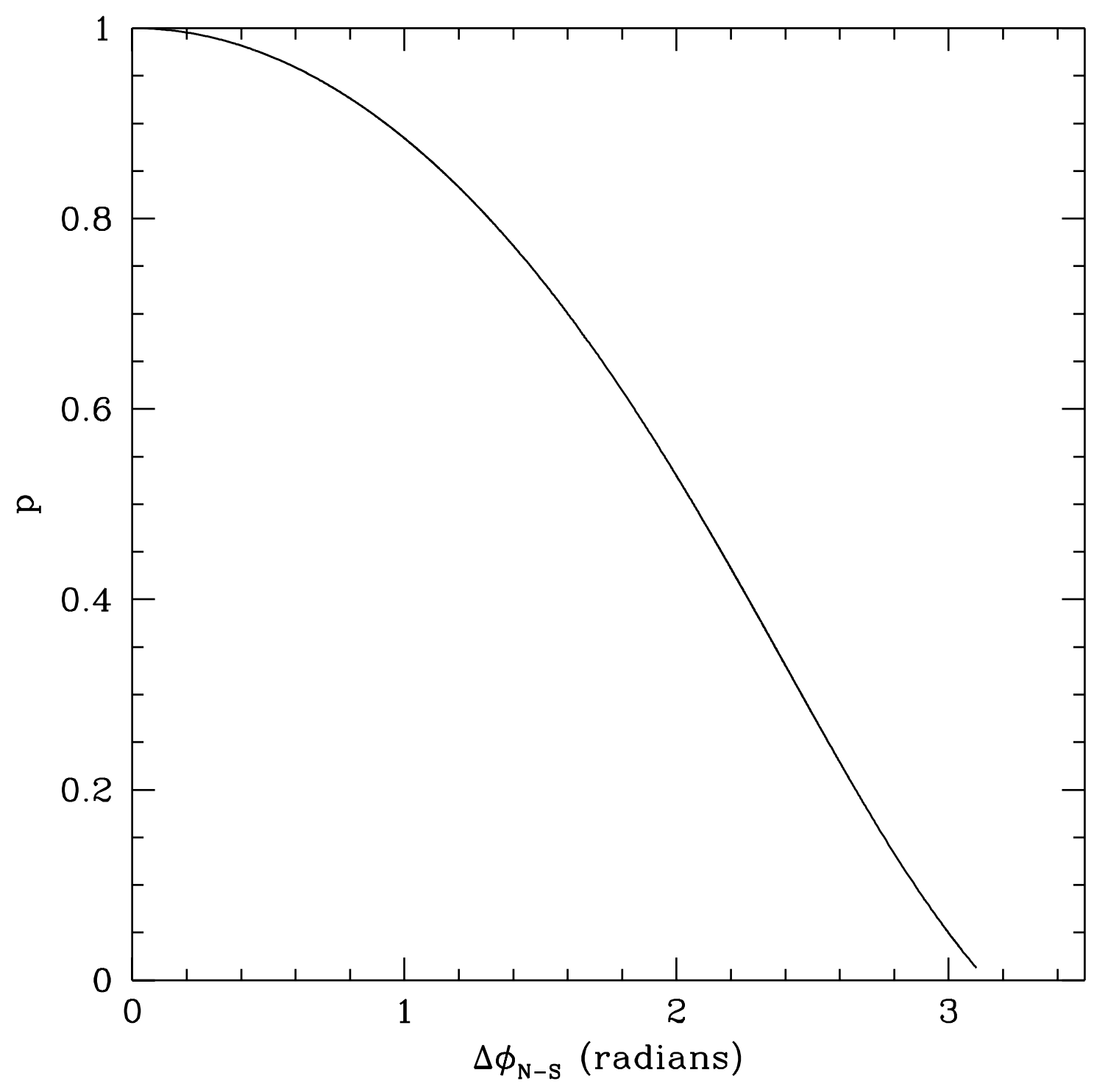

Fig. 2.- The radial index $p$ of the magnetic flux function $\mathcal{P}$ (eq. [3]) is plotted versus the net twist angle $\Delta \phi_{\mathrm{N}-\mathrm{S}}=\Delta \phi(\theta \rightarrow 0)$ between the north and south magnetic poles (eq. [B]). 

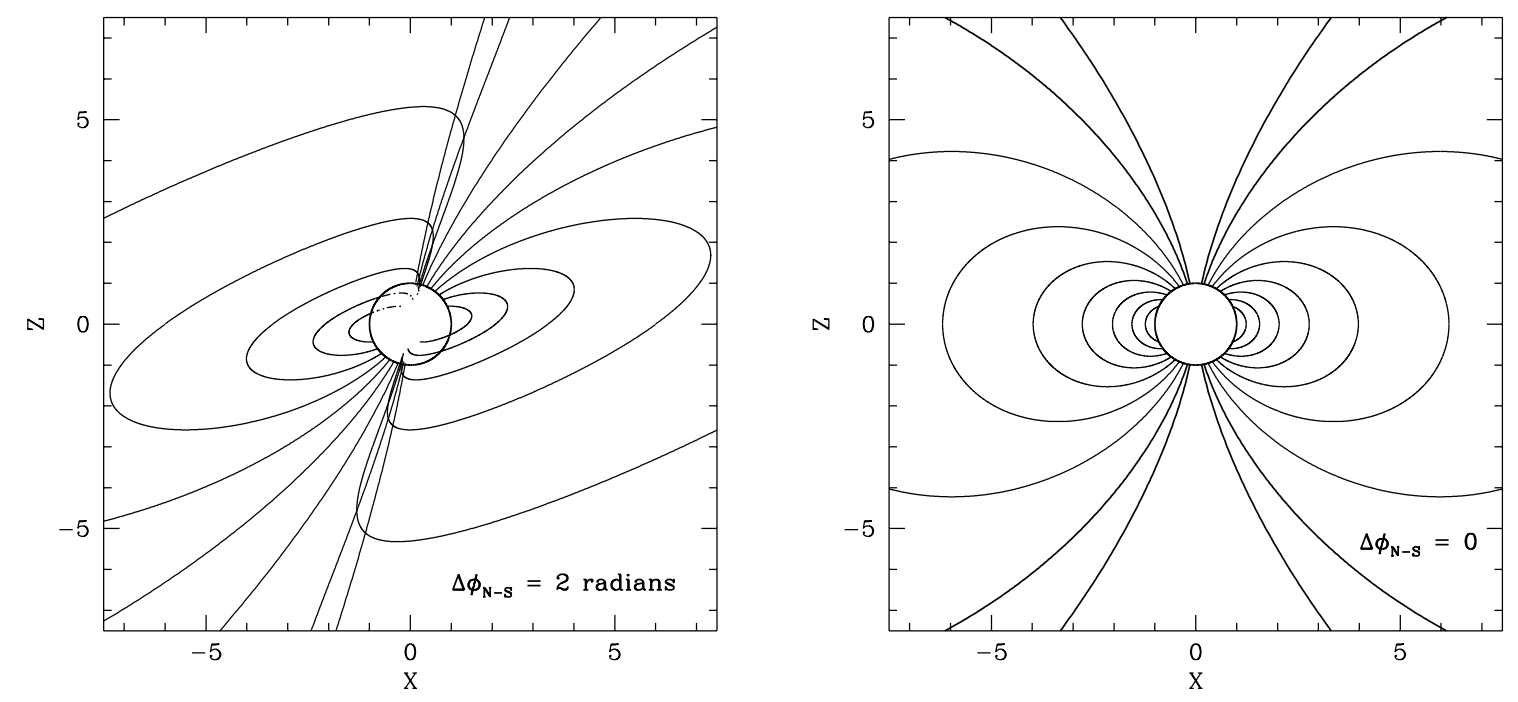

Fig. 3.- An example of a twisted, self-similar, force-free magnetosphere, with net twist angle $\Delta \phi_{\mathrm{N}-\mathrm{S}}=2$ radians. Only a small number of field lines are plotted here. The field lines protruding from the top right and bottom left corners of the star are anchored in the $X-Z$ plane, at regular intervals $\Delta \mu=0.1$. Dashed lines indicate that the field is projected behind the star. A pure dipole is shown for comparison. 


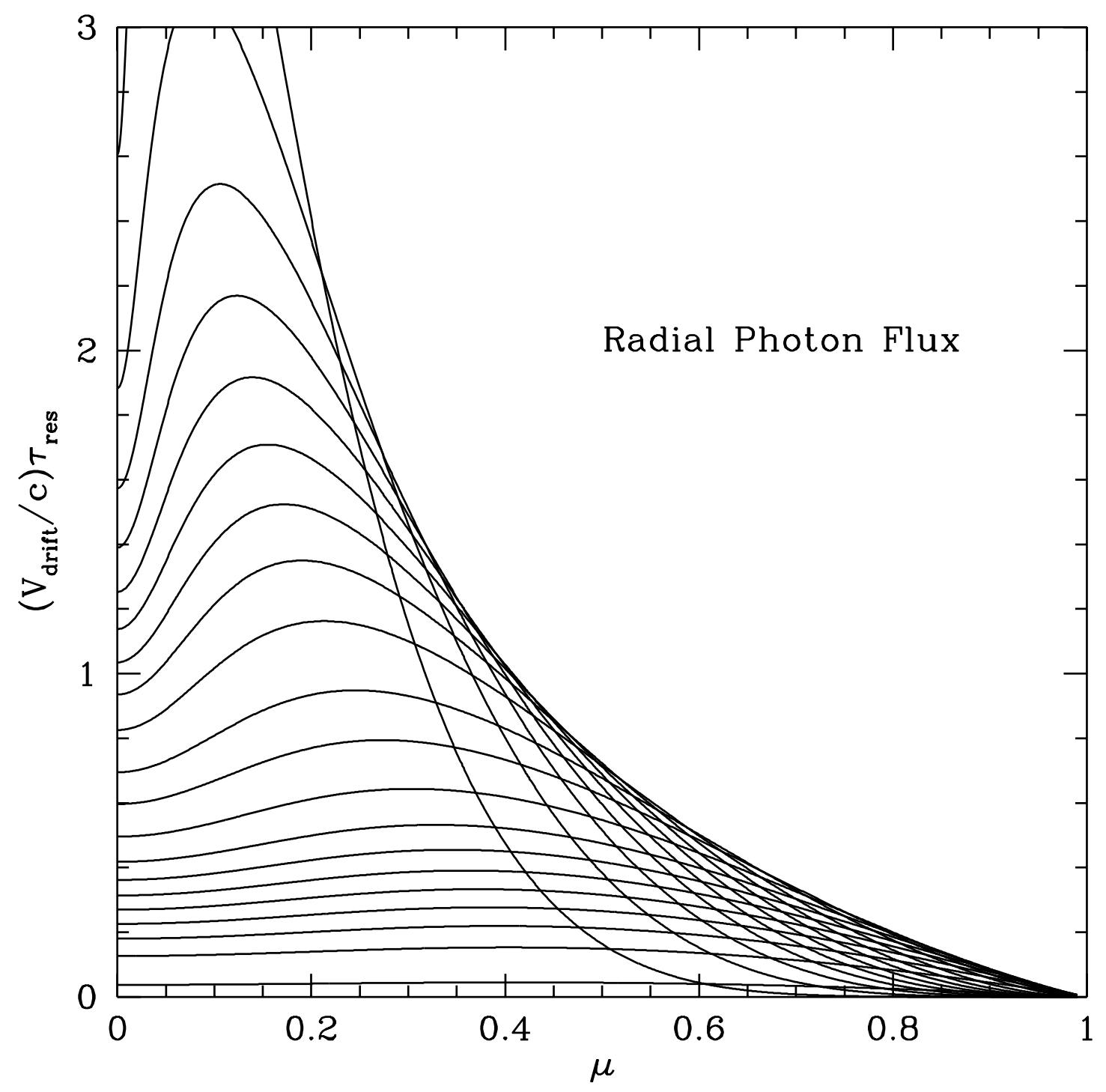

Fig. 4.- Optical depth $\tau_{\text {res }}$ to scattering at the cyclotron resonance (eq. 24]) experienced by radially streaming photons, as a function of magnetic co-latitude $\mu=\cos \theta$ and the drift speed $V_{\text {drift }}$. This optical depth is calculated in the approximation that the charges are static. The various curves correspond to different twist angles $\Delta \phi_{\mathrm{N}-\mathrm{S}}$; the peak value of $\tau_{\text {res }}\left(V_{\text {drift }} / c\right)$ is $\sim 0.6$ when the twist is $\Delta \phi_{\mathrm{N}-\mathrm{S}}=1.5$ radians. Within a self-similar twisted magnetosphere, this optical depth is independent of the radius, frequency, or the charge/mass ratio of the scattering particle (as long as the cyclotron resonance sits outside the spherical surface in which the magnetic field is anchored). If the current is supported by more than one species of charge carrier, as is generally the case, the plotted value of $\tau_{\text {res }}$ must be multiplied by the fraction of the current carried by the species of scattering charge. 


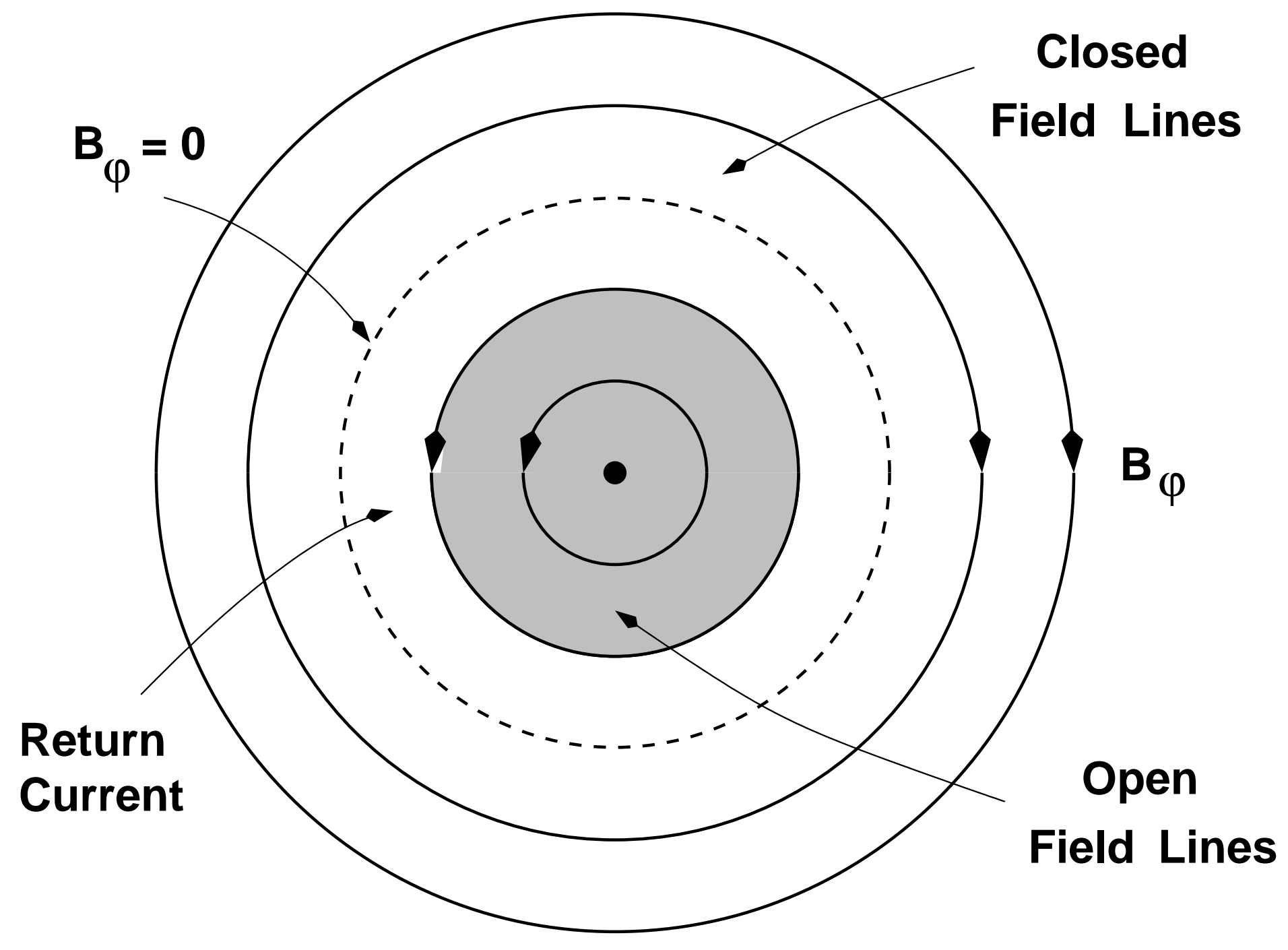


Fig. 5.- In one magnetic hemisphere, a space-charge limited flow will generate a current of the opposite sign to the current flowing on the closed, twisted field lines. The toroidal component of the field will then have an opposing sense on the open and closed field lines. In the absence of pairs, this may cause a cancellation of the twist in the intermediate regions of the magnetosphere. An electron-positron pair cascade allows the density of currentcarrying particles to build up, so that the Goldreich-Julian charge density $-\boldsymbol{\Omega} \cdot \mathbf{B} / 2 \pi c$ can be maintained even as the current flows backward compared with a pure space-charge limited flow. 


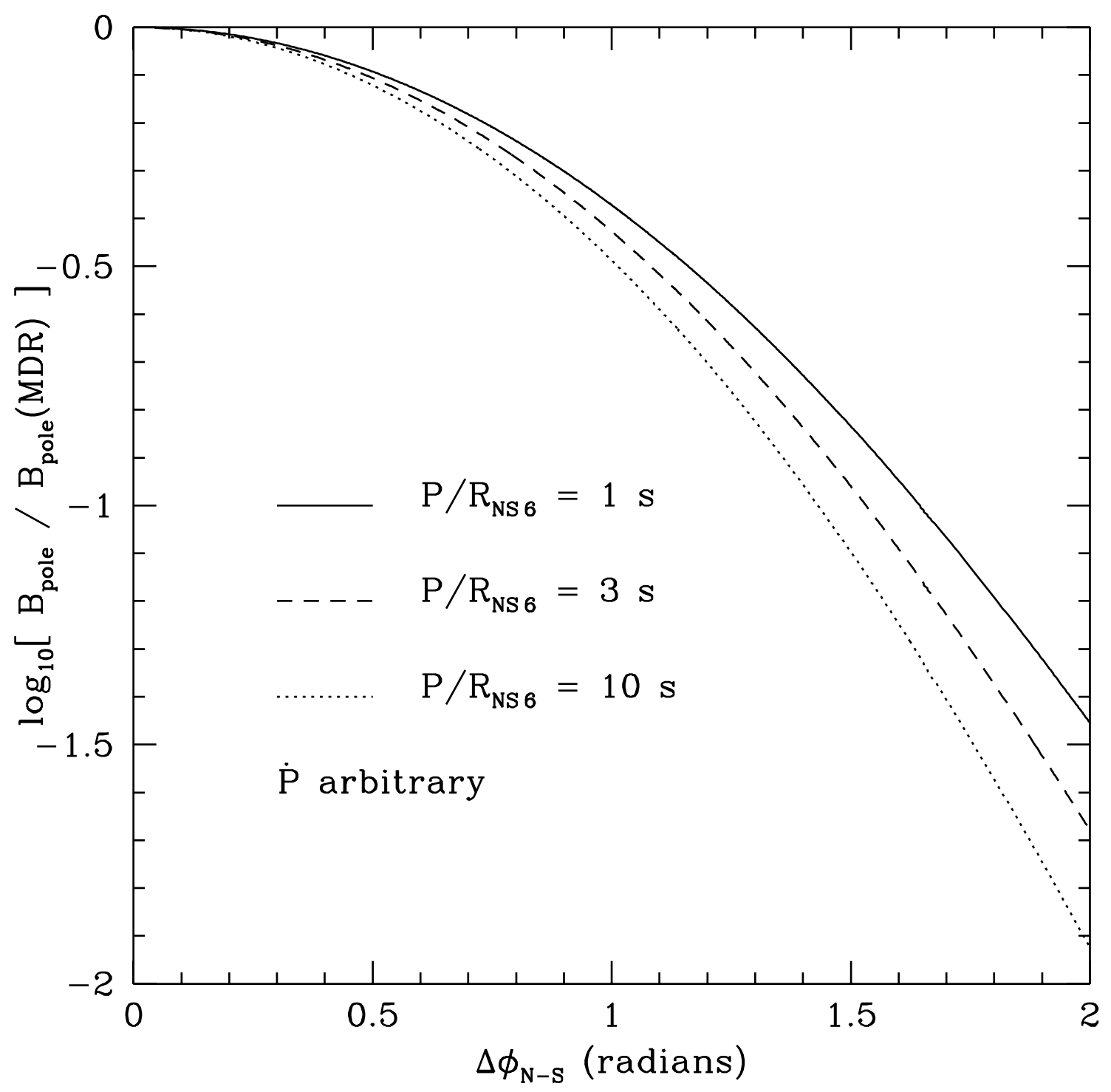

Fig. 6.- Polar surface magnetic field, as inferred from a measured spin period $P$ and period derivative $\dot{P}$, compared with the classical magnetic dipole formula (eq. 444) evaluated at $\sin \alpha=1$. 


\title{
Electrodynamics of Magnetars: \\ Implications for the Persistent X-ray Emission and Spindown of the Soft Gamma Repeaters and Anomalous X-ray Pulsars
}

\author{
C. Thompson ${ }^{1}$, M. Lyutikov ${ }^{2,3,4}$, and S.R. Kulkarni ${ }^{5}$ \\ Submitted to the Astrophysical Journal
}

\begin{abstract}
We consider the structure of neutron star magnetospheres threaded by large-scale electrical currents, and the effect of resonant Compton scattering by the charge carriers (both electrons and ions) on the emergent X-ray spectra and pulse profiles. In the magnetar model for the Soft Gamma Repeaters and Anomalous X-ray Pulsars, these currents are maintained by magnetic stresses acting deep inside the star, which generate both sudden disruptions (SGR outbursts) and more gradual plastic deformations of the rigid crust. We construct self-similar force-free equilibria of the current-carrying magnetosphere with a power law dependence of magnetic field on radius, $\mathbf{B} \propto r^{-(2+p)}$, and show that a large-scale twist of field lines softens the radial dependence of the magnetic field to $p<1$. The spindown torque acting on the star is thereby increased in comparison with an orthogonal vacuum dipole. We comment on the
\end{abstract}

\footnotetext{
${ }^{1}$ Canadian Institute for Theoretical Astrophysics, 60 St. George St., Toronto, ON M5S 3H8

${ }^{2}$ Department of Physics, McGill University, Montréal, QC

${ }^{3}$ Massachusetts Institute of Technology, 77 Massachusetts Avenue, Cambridge, MA 02139

${ }^{4}$ CITA National Fellow

${ }^{5}$ California Institute of Technology, 105-24, Pasadena, CA 91125
} 
strength of the surface magnetic field in the SGR and AXP sources, as inferred from their measured spindown rates, and the implications of this model for the narrow measured distribution of spin periods.

A magnetosphere with a strong twist $\left(B_{\phi} / B_{\theta}=O(1)\right.$ at the equator) has an optical depth $\sim 1$ to resonant cyclotron scattering, independent of frequency (radius), surface magnetic field strength, or charge/mass ratio of the scattering charge. When electrons and ions supply the current, the stellar surface is also heated by the impacting charges at a rate comparable to the observed X-ray output of the SGR and AXP sources, if $B_{\text {dipole }} \sim 10^{14} \mathrm{G}$. Redistribution of the emerging X-ray flux at the cyclotron resonance will strongly modify the emerging pulse profile and, through the Doppler effect, generate a non-thermal tail to the X-ray spectrum. We relate the sudden change in the pulse profile of SGR 1900+14 following the 27 August 1998 giant flare, to an enhanced optical depth at the electron cyclotron resonance resulting from a sudden twist imparted to the external magnetic field during the flare. The self-similar structure of the magnetosphere should generate frequency-independent profiles; more complicated pulse profiles may reflect the presence of higher multipoles, ion cyclotron scattering, or possibly non-resonant Compton scattering of O-mode photons by pair-loaded currents.

Subject Headings: gamma rays: bursts - stars: neutron - X-rays: stars

\section{Introduction}

Our view of neutron stars has been strongly influenced by radio pulsars. The demographics and physical properties of isolated neutron stars have largely been derived from extensive pulsar searches, and associations of pulsars with supernovae. Over the last decade, thanks to new high energy missions, there has been a growing recognition of diversity in the neutron star population. Considerable attention has been focused on the radio-quiet neutron stars (RQNS), which often have a bright presence in the X-ray sky (Frail 1998). Although the known RQNS are certainly heterogeneous, two groups of objects seem to show sufficient regularity to classify them: the Anomalous X-ray pulsars (AXPs) and the Soft Gamma-ray Repeaters (SGRs). Both appear to be young objects given their location within supernova remnants and star-forming regions.

The AXPs have spin periods in the range $P=6-12 \mathrm{~s}$, characteristic ages $P / \dot{P}=3 \times 10^{3}-4 \times 10^{5} \mathrm{yr}$, and X-ray luminosities $L_{X}=5 \times 10^{34}-10^{36} \mathrm{erg} \mathrm{s}^{-1}$ (for recent reviews see Mereghetti 2000, Thompson 2001). Three out of the six AXPs lie close 
to the centers of SNRs, confirming their youth. In none of these objects is there evidence of a binary stellar companion, or the usual indicators of accretion through a disk. Their spin-down luminosity is always less than a few percent of the observed X-ray luminosity. The unfamiliar source of energy makes these objects anomalous.

SGRs are identified by their hard X-ray flares. Once localized, their quiescent X-ray emission (also in the range $L_{X} \sim 5 \times 10^{34}-10^{36} \mathrm{erg} / \mathrm{s}$ ) allows further studies of these equally enigmatic objects. SGRs have spin periods in the range 5-8 s but somewhat smaller characteristic ages than the AXPs, less than a few thousand years. Only one or two of the four SGRs are concident with a SNR. However, all the SGRs are found in star-forming regions, attesting to their youth. It is important to note that only $50 \%$ of pulsars with characteristic ages less than $\sim 5 \times 10^{4}$ years have associated SNRs. Thus, for both SGRs and AXPs, the frequency of associations with SNRs appears no different statistically from that of young pulsars (Gaensler et al. 2001). As with AXPs, there are no observational reasons to believe that these objects have companions; nor evidence for accretion. The reader is referred to Hurley (2000) and Thompson (2000) for recent reviews of the SGRs.

The giant flare of 5 March 1979 (Mazets et al. 1979) now associated with SGR 0525-66 provided the first observational evidence that the SGRs are highly magnetized neutron stars or "magnetars". These objects where hypothesized to form through dynamo action in supernova collapse (Duncan \& Thompson 1992). Superstrong magnetic fields, $B>10^{15} \mathrm{G}$, are able to supply enough energy to power the bursts, thereby matching the extreme peak luminosities of more a million times Eddington; and to confine a significant proportion of the cooling plasma over the long (> $200 \mathrm{~s}$ ) duration of the flare (Duncan \& Thompson 1992; Pacyzński 1992; Thompson \& Duncan 1995). The presence of 8-s pulsations in the March 5 burst provided a consistent and independent support for the magnetar hypothesis.

The discovery of long period pulsations in the quiescent X-ray emission of SGRs by Kouveliotou et al. (1998) has opened a rich field of timing studies of SGRs (Woods et al. 1999c, 2001b; Kaspi et al. 1999, 2001; Gavriil \& Kaspi 2001), from which we are now able to obtain critical information about both the electrodynamics and the superfluid behavior of these interesting objects, in much the same way as has been the case with timing studies of radio pulsars.

The same magnetar model which has successfully described the bright outbursts of the SGRs is also able to account for their long spin periods and their quiescent emission (through magnetic field decay). Indeed, the SGRs in their quiescent states overlap with the AXPs in a three-dimensional parameter space $\left(P, \dot{P}\right.$ and $\left.L_{X}\right)$. This striking similarity motivated the suggestion that they share a common energy source: the decay of a very strong magnetic field (Thompson \& Duncan 1996, hereafter TD96). The bursting activity 
of the SGRs is highly intermittent, so that some of the AXPs may be dormant SGRs.

The circumstantial connection between the SGRs and AXPs has become stronger with two objects SGR 0526-66 and AXP 1E 1048.1-5937 constituting as possible "missing links'. After the 5 March 1979 giant flare, SGR 0526-66 continued to emit a dozen shorter bursts, but has not been an active burster since 1983. Its persistent spectrum is consistent with a pure power law with photon index $15=3.5$ (Kulkarni et al. 2001). This relatively soft power-law index is characteristic of the AXPs, but is much softer than the $\Gamma \simeq 2.2$ measured in the persistent emission of the other SGRs. By contrast, 1E 1048.1-5937 has never been observed to burst, but its power-law index $\Gamma=2.5$ is harder than that of SGR 0526-66. Equally significantly, this object appears to have the largest timing noise of AXPs and at levels similar to those of SGRs (Kaspi et al. 2001). Specifically, between 1994 and 1996, the spindown torque of 1E 1048.1-5937 appears to have increased by a factor $\sim 2$ over its long term value (Paul et al. 2000). This behavior is similar to the accelerating spindown observed in SGR 1900+14 several months following the August 27 giant flare (Woods et al. 2001b). Thus, the latest timing results are unveiling a remarkable similarly in the detailed spin behavior of the SGRs and AXPs.

Within the combined population of SGR and AXP sources, there is overall a positive correlation between spindown rate and the hardness of the spectral index $\Gamma$ in the persistent emission (Marsden \& White 2001). There is, similarly, a positive correlation between hardness and pulsed fraction within the AXP population (e.g. Table 2 in Kaspi et al. 2001).

This smooth continuity of the X-ray and timing properties, when combined with the other observational similarities summarized above, provides a strong phenomenological link between SGRs and AXPs. If the SGRs are magnetars, then so are the AXPs.

The discovery of optical counterparts of AXPs (Hulleman, van Kerkwijk \& Kulkarni 2000; Hulleman et al. 2001) offers new clues about AXPs. The existing optical data on SGRs are consistent with SGR counterparts being similarly faint (Kaplan et al. 2001a). These authors find that the dimness of the optical counterpart is incompatible with standard accretion disk models and thus by elimination provide evidence for the magnetar model.

There do, however, exist significant differences between AXPs and SGRs. Beyond the obvious difference - the spectacular X-ray outbursts of the SGRs - there is also a puzzling discrepency between different methods of estimating their relative ages. The location of three AXPs close to the centers of their SNRs would suggest that - given similar proper

\footnotetext{
${ }^{6}$ Here $d N / d E \propto E^{-\Gamma}$.
} 
motions - the SGRs must descend from the AXPs. However, the characteristic ages of SGRs are typically smaller than those of AXPs. Combining these two observations, one infers that the measured spindown of an SGR must be transiently accelerated compared with its long-term average. Precisely this effect is now being observed (over relatively brief 1-2 year intervals) in the timing solutions (Paul et al. 2000; Woods et al. 2001b). The positive correlation between spindown rate and cumulative bursting activity further suggests that activity as an SGR source is also intermittent. One of the basic goals of this paper is to connect this apparent transient behavior to the magnetar model.

This general picture does not require a temporal link between the SGRs and AXPs, but if one exists then the SGRs are probably old AXPs which hibernate most of the time and display SGR-like activity over a small fraction $(\sim 10-25 \%)$ of their lifetime. Alternatively, some AXPs may never emit bright X-ray outbursts, and may be distinguished from the SGRs in some hidden attribute, e.g., the multipolarity of the magnetic field.

How do the AXPs and SGRs fit into the grander picture of neutron star behavior? Recent radio surveys have discovered pulsars with polar magnetic fields approaching $10^{14} \mathrm{G}$ (Camilo 2000), continuous with the lower range of fields deduced from AXP spindown. However, these objects appear to be no different from other radio pulsars and in particular are not X-ray bright (Pivovaroff et al. 2000). This bifurcation in X-ray behavior suggests that the magnetar phenomenon (e.g. rapid magnetic field decay) does not turn on until $B$ exceeds $10^{14} \mathrm{G}$. In particular, AXPs and radio pulsars may be distinguished by the strength of the internal magnetic field.

In this paper, we present a physical model which synthesizes the persistent emission and torque behavior of the SGRs and AXPs, relates that behavior to the mechanism driving the SGR flares, and provides a strong suggestion that these objects were born with very rapid rotation. The starting hypothesis is that the magnetic field is globally twisted inside the star - up to a strength about 10 times the external dipole - and is strong enough to twist up the external field (at intervals). In the magnetar model, these currents provide the most promising mechanism for generating the non-thermal persistent emission, through magnetospheric Comptonization and surface heating (TD96). Direct evidence for this effect comes from the observed change in the pulse profile of SGR 1900+14 following the 27 August 1998 giant flare (Thompson et al. 2000). We explain how these persistent currents flowing outside the star will heat its surface, and modify the emerging X-ray spectrum and pulse profile. The external twist will also increase the electrical current flowing across the speed of light cylinder, and therefore the spindown torque acting on the star. A positive correlation between spectral hardness and spindown rate is a natural consequence of this model. 
The presence of an ultrastrong magnetic field, $B \sim 4 \times 10^{15} \mathrm{G}$, in the deep interior of the SGR and AXP sources has a few further interesting consequences.

First, such a strong field can comfortably account for the total energy released by these sources in various channels. Not only do magnetars lose energy through electromagnetic emissions (X-ray, UV and optical), but also through relativistic particles and neutrino radiation from the deep crust and core. There is evidence for particle losses during the 27 August 1998 giant burst of SGR 1900+14 (Frail, Kulkarni \& Bloom 1999). While current observations do not show any plerions around other SGRs (or AXPs), the upper limits on the persistent particle luminosity obtained from these observations are not constraining. If the observed X-ray output of the AXPs is dominated by transport of the magnetic field through the neutron star core, then neutrino losses will exceed the surface flux by a factor $\sim 30$ (TD96; Heyl \& Kulkarni 1998). Estimating the bolometric losses is also complicated in some sources (primarily the AXPs) by a steep power-law component in the X-ray spectrum, which is not observed below $\sim 0.5 \mathrm{keV}$ due to absorption. Assuming a typical observed luminosity of $3 \times 10^{35} \mathrm{erg} \mathrm{s}^{-1}$ and a mean age of $10^{4} \mathrm{yr}$ we obtain $E \sim 10^{47} \mathrm{erg}$, comparable to the inferred output of the bursting SGR sources. This should be compared to the total magnetic energy of $10^{47} B_{15}^{2}$ erg where $B_{15}$ is the strength of the field in units of $10^{15} \mathrm{G}$ and it is assumed that this field permeates the entire volume of the neutron star. The very high electrical conductivities of neutron star interiors will prevent currents from being entirely dissipated, and so internal fields somewhat stronger than $\sim 10^{15} \mathrm{G}$ are needed to supply the entire bolometric output of an AXP or SGR source. This simple estimate also underlines the importance of obtaining more precise measurements of the low-energy spectral cutoff in sources with soft spectra.

Second, the presence of ultrastrong internal magnetic fields leads to a simple physical mechanism for the termination of the bright X-ray emissions of the SGR and AXP sources. It is clear observationally that the AXP/SGR phenomenon is restricted to young objects, with total ages less than $10^{4}-10^{5} \mathrm{yr}$. What causes this decline in magnetar activity? One explanation involves the strong temperature-dependence of the rate of magnetic field transport through the neutron star core (e.g. Goldreich \& Reisenegger 1992). Transport is dramatically accelerated by internal heating, and so the transport of a deeply anchored field will cut off sharply when cooling of the star is dominated by surface X-ray emission (TD96).

What do old magnetars look like? It is in this context that the discovery of long period isolated nearby X-ray pulsars such as RX J0720.4-3125 (Haberl et al. 1997) becomes interesting. There are three such objects (see Treves et al. 2000 for a review), a high fraction $(30 \%)$ of nearby neutron stars. Several authors have argued these objects to be old magnetars (e.g. Heyl \& Hernquist 1998, Kulkarni \& van Kerkwijk 1998). If indeed 
this is the case, then the birthrate of magnetars is a significant fraction of the pulsar birth rate. Furthermore, the similarity of the periods of these objects with those of AXPs+SGRs indicates that period evolution may slow down after the active magnetar phase.

Third, as we have already mentioned, the presence or absence of a strong toroidal field offers an explanation for the bifurcation in behavior between radio pulsars and the X-ray bright SGRs and AXPs. More generally, the maximum total (as opposed to dipolar) field which will manifest pulsar-like activity (and the minimum field which is needed to power an AXP or SGR) needs a more detailed theoretical explanation. These minima and maxima need not coincide. Is there a gap between the B-field strengths of the observed high-field pulsars, and the magnetar population? The theoretical model advanced in this paper will be useful in making this question more precise.

\subsection{Plan of the Paper}

In this paper, we consider the properties of neutron stars whose external magnetic fields support global electrical currents, flowing across a large fraction of the stellar surface. We construct axisymmetric, self-similar solutions to the force-free equation, which describe the structure of a magnetosphere with a global twist between the two hemispheres. We begin in Section 2 with a general discussion of how the decay of an electrical current flowing inside stellar material of a very high electrical conductivity may be accelerated as hydromagnetic stresses divert part of the current to the exterior, where it is damped much more rapidly. Section 3 describes the construction and basic properties of our self-similar model, which forms a one-parameter sequence labeled by the net angle through which the magnetic field lines are twisted (or, equivalently, by the radial power-law index of the magnetic field). The modification of the corotation charge density by static currents is calculated, and the matching of our twisted solution onto open magnetic field lines is discussed qualitatively.

Resonant cyclotron scattering of photons by the charge carriers is explored in Section 4 , where the following remarkable property is demonstrated: the optical depth to resonant scattering is proportional to the twist, but does not depend explicity on the radius, or the mass and electrical charge of the current-carrying particles. This resonant optical depth is largest at the magnetic equator, but decreases to zero near the magnetic axis (where the current flowing along more extended field lines is relatively weak).

Section 5 gives an introduction to how the X-ray spectrum and pulse profile of a magnetar will be modified by multiple resonant scattering in the magnetosphere, as well as the implication for the mechanism of the giant flares in the SGR sources. In Section 6, 
we calculate how the surface field inferred from spindown measurements is modified, and then consider the implications of our model for the narrow distribution of pulse periods measured in the SGR and AXP sources. Section 7 closes the paper with a summary of our findings, and their more general implications for the nature of the SGR and AXP sources.

\section{Twisted Neutron Star Magnetospheres}

The non-thermal persistent emission of the SGRs has been ascribed to a static twist imparted to the external magnetic field by sub-surface motions during X-ray flares, with the effect of diverting an electrical current from the interior of the star to its exterior (Thompson et al. 2000). Magnetic fields exceeding $\sim 10^{14} \mathrm{G}$ are strong enough to fracture the deep crust of a neutron star and, if stronger than $\sim 10^{15} \mathrm{G}$, will undergo rapid transport through the dense stellar interior over the short $\sim 10^{4}-10^{5} \mathrm{yr}$ active lifetime of the SGR/AXP sources (TD96; Heyl \& Kulkarni 1998).

The magnetic fields of neutron stars are most likely generated by a hydromagnetic dynamo as the star is born. At this time, the star undergoes a violent convective instability driven by neutrino cooling (Burrows 1987; Keil, Janka, \& Mueller 1996). Magnetic fields are easily transported across the boundary of the neutron-rich core, where they can reconnect from the field anchored in the star (Thompson \& Murray 2001). In this manner, net magnetic helicity builds up in the anchored field as the disconnected field carries away helicity. A neutron star is generically formed with strong differential rotation (because its equation of state is stiff), and will support a large-scale helical dynamo when its initial spin period is shorter than the convective overturn time of $\sim 3-10$ ms (Duncan \& Thompson 1992).

Consider a twisted magnetic field which is anchored in a highly conducting crust of a neutron star (Fig. 11). Suppose that initially the twist vanishes outside the star, where the conductivity is much lower. Then the current closes through a thin surface layer, which feels a Lorentz force $\frac{1}{c} \mathbf{J} \times \mathbf{B}$. In the absence of any tensile strength, this force causes the surface layer and the external field to twist up. The net effect is to distribute the twist more uniformly along the flux tube (Fig. 1), and therefore to reduce its total energy. The decay of the current is accelerated by forcing it into a medium of lower conductivity the magnetosphere. The twist will then relax in a time comparable to the decay time

$t_{\text {decay }}^{\text {ex }}$ outside the star. In the case of a realistic neutron star, this process will be impeded by the compositional stratification of the stellar core: the untwisting of the magnetic field is suppressed if it requires the motion of fluid elements across equipotential surfaces (Thompson \& Duncan 2001). 
In the SGRs and AXPs, such an external current can be supported by charges stripped from the surface: the effective temperature is high enough to allow thermal emission of electrons and light ions (hydrogen and helium), even in the presence of a $\sim 10^{15} \mathrm{G}$ magnetic field (Thompson et al. 2000). Any large-scale deformation of a neutron star is further constrained by the rigidity of its crust. A weak magnetic field is pinned by the crust, and the external current decays without being replenished. The behavior of magnetic fields stronger than $\sim 10^{14}-10^{15} \mathrm{G}$ depends on the intermittency with which the crust responds to the applied magnetic stresses. The compositional stratification of a neutron star (Reisenegger \& Goldreich 1992) strictly limits the degree to which an internal magnetic field can unwind promptly. The lowest energy deformations of the rigid crust are rotational, and large-scale fractures can be triggered repeatedly as transport processes (such as ambipolar diffusion and Hall drift) allow the shear components of the magnetic stress to build up with time. An empirical estimate of this growth time is given by the mean duration $\Delta t_{\text {flare }} \sim 10^{2} \mathrm{yr}$ between giant flares in any one SGR source (e.g. Mazets et al. 1999). The external current will be maintained more or less continuously if $t_{\text {decay }}^{\text {ex }} \gtrsim t_{\text {growth }} \sim \Delta t_{\text {flare}}$; otherwise, the external current will decay in between spasmodic events. More gradual, plastic deformations of the star are also possible and, in the magnetar model, are required to explain measured variations in the X-ray output of some AXPs (e.g. Iwasawa, Koyama, \& Halpern 1992) which are not associated with bright X-ray bursts. In this case, a non-potential magnetic field will be maintained outside the star only if $t_{\text {decay }}^{\text {ex }} \gtrsim t_{\text {growth }}$. Note also that the net twist which is maintained along extended field lines (reaching out beyond several stellar radii) is controlled by the current flowing through only a fraction of the surface.

\section{Force-Free Equilibria}

The exterior of an isolated neutron star is traditionally modeled as a potential magnetic field, excluding a narrow bundle of field lines which extend out to the speed of light cylinder (Pacini 1967; Goldreich \& Julian 1969; Ruderman \& Sutherland 1975). We generalize this classical dipole model to include an electrical current flowing continuously across the entire surface of the star. We assume initially that the star does not rotate, so that the closed magnetic field lines fill an infinite volume outside the star. The effects of slow rotation are discussed in Sections 3.1 and 3.2 .

The energy density of the charge carriers needed to supply the current is, in this context, tiny compared with $B^{2} / 8 \pi$, and the bare space charge $|\rho| \ll J / c$ on the closed field lines. This leads us to solve the force-free equation, $\mathbf{J} \times \mathbf{B}=0$, to determine the structure

of the magnetic field around a highly conducting spherical mass of radius $R_{\mathrm{NS}}$. The solution 
of this equation can be written formally as

$$
\nabla \times \mathbf{B}=\alpha(\mathcal{P}) \mathbf{B}
$$

When the magnetosphere is axisymmetric (as we will assume), the magnetic field lines form a one-dimensional sequence labeled by the flux parameter $\mathcal{P}=\mathcal{P}(R, \theta)$. The poloidal magnetic field can be written as

$$
\mathbf{B}_{P}=\frac{\nabla \mathcal{P} \times \hat{\phi}}{R \sin \theta} .
$$

(In components, $B_{R}=-R^{-2} \partial \mathcal{P} / \partial(\cos \theta)$ and $B_{\theta}=-(R \sin \theta)^{-1} \partial \mathcal{P} / \partial R$.) The constancy of the current along an infinitesimal bundle of magnetic flux guarantees that the coefficient $\alpha$ is a function only of $\mathcal{P}$.

As a major simplification we search for self-similar configurations

$$
\mathcal{P}=\mathcal{P}_{0} r^{-p} F(\cos \theta)
$$

adapting the trick of Lynden-Bell and Boily (1994) who studied force-free equilibria bounded by an infinite, conducting plane. Here, $r=R / R_{\mathrm{NS}}, \theta$ is the magnetic co-latitude, and $\mathcal{P}_{0}=\frac{1}{2} B_{\text {pole }} R_{\mathrm{NS}}^{2}$. The resulting sequence of magnetospheric equilibrium which is essentially identical to that obtained previously, in particular cases, by Wolfson (1995). Comparing with equation (11) shows that $\alpha(\mathcal{P})$ is proportional to $\mathcal{P}^{1 / p}$, and on dimensional grounds one can write

$$
\alpha(\mathcal{P})=\frac{C^{1 / 2}}{R_{\mathrm{NS}}}\left(\frac{p+1}{p}\right)^{1 / 2}\left(\frac{\mathcal{P}}{\mathcal{P}_{0}}\right)^{1 / p} .
$$

The shape of the field lines, including the radial index $p$, is determined uniquely by the single parameter $C$ which is related to the strength of the current. The poloidal components of eq. (II) can be integrated to give

$$
B_{\phi}=\frac{\int \alpha(\mathcal{P}) d \mathcal{P}}{R \sin \theta}=\frac{p}{p+1} \frac{\mathcal{P} \alpha(\mathcal{P})}{R \sin \theta} .
$$

Substituting eqs. (22)-(5) into the $\phi$-component of eq. (11) then gives the non-linear equation

$$
p(p+1) F+\left(1-\mu^{2}\right) \frac{\partial^{2} F}{\partial \mu^{2}}=-C F^{1+2 / p}
$$

for the angular factor $F=F(\mu)$.

The solution of eq. (6), including the dependence $p(C)$, is uniquely defined by the parameter $C$ and by the three boundary conditions: $B_{R} \propto F^{\prime}=0$ at $\mu \equiv \cos \theta=0$ (the magnetic equator), $F^{\prime}=$ const $=-2$ at $\mu=1$ (corresponding to a fixed flux density 
$B_{\text {pole }}$ at the magnetic pole), and $B_{\phi}(\mu=1) \propto F(1)=0$. (Wolfson 1995 instead fixes the magnetic flux threading each hemisphere.) The solutions are symmetric under $\mu \leftrightarrow-\mu$, with $F(\mu)=1-\mu^{2}$ representing a pure dipole. For each value of $C \leq 0.873$ there are two solutions for $p$. The upper branch connects continuously to the vacuum dipole $C=0$, $p=1\left(B_{R}, B_{\theta} \propto R^{-3}\right)$; and the lower branch connects to the split monopole $C=0, p=0$ $\left(B_{R}= \pm 2 \mathcal{P}_{0} / R^{2}\right)$.

Every twisted force-free magnetosphere has a finite toroidal field

$$
\frac{B_{\phi}(\theta)}{B_{\theta}(\theta)}=\left[\frac{C}{p(1+p)}\right]^{1 / 2} F^{1 / p}(\theta)
$$

A magnetic field line anchored at polar angle $\theta$ will twist through a net angle

$$
\Delta \phi(\theta)=2 \int_{\theta}^{\pi / 2} \frac{B_{\phi}(\theta)}{B_{\theta}(\theta)} \frac{d \theta}{\sin \theta}
$$

before returning to the stellar surface. Both branches of force-free equilibria connect to form a one-parameter sequence, labeled by the net twist of field lines anchored close to the two magnetic poles, $\Delta \phi_{\mathrm{N}-\mathrm{S}} \equiv \Delta \phi(\theta \rightarrow 0)$. For modest twists $\Delta \phi_{\mathrm{N}-\mathrm{S}} \lesssim 1$ one has

$$
\Delta \phi_{\mathrm{N}-\mathrm{S}} \simeq 2\left(\frac{B_{\phi}}{B_{\theta}}\right)_{\theta=\pi / 2}
$$

The radial index $p$ is a decreasing monotonic function of $\Delta \phi_{\mathrm{N}-\mathrm{S}}$ (Fig. 2). The radial dependence of the magnetic field softens to $\mathbf{B} \propto r^{-2.88}$ when $\Delta \phi_{\mathrm{N}-\mathrm{S}}=1$ radian. The net twist approaches $\Delta \phi_{\mathrm{N}-\mathrm{S}}=\pi$ (one-half turn) in the split monopole limit $(p=0)$. For comparison, a twisted cylindrically symmetric magnetic field pinned in an infinite half-plane expands to infinity after $1 / \sqrt{3}$ turns (Lynden-Bell \& Boily 1994). One example of a twisted, self-similar magnetosphere (corresponding to $\Delta \phi_{\mathrm{N}-\mathrm{S}}=2$ radians) is depicted in Fig. 3 .

These solutions to the force-free equation carry a net helicity

$$
\mathcal{H}_{B}=\int \mathbf{A} \cdot \mathbf{B} d V=\frac{3 \pi}{2}\left(B_{\text {pole }} R_{\mathrm{NS}}^{2}\right)^{2} \sqrt{\frac{C}{p(p+1)}} \int_{0}^{1} d \mu \frac{[F(\mu)]^{2+1 / p}}{1-\mu^{2}}
$$

This expression reduces to

$$
\mathcal{H}_{B} \simeq \frac{2 \pi}{5}\left(B_{\text {pole }} R_{\mathrm{NS}}^{2}\right)^{2} \Delta \phi_{\mathrm{N}-\mathrm{S}}
$$

for modest twists $\Delta \phi_{\mathrm{N}-\mathrm{S}} \lesssim 1$ radian. The helicity is approximately $\mathcal{H}_{B} \sim B_{\phi} B_{\theta} R^{4}$, and can be expressed in terms of the magnetic energy as $\mathcal{H}_{B} \sim\left(B_{\phi}^{2} R^{3}\right) \times R$ when the twist is moderately large, $B_{\phi} \sim B_{\theta}$. This means that the magnetic energy is minimized at fixed 
helicity if the helicity is concentrated close to the star. Indeed, the current density decreases toward the magnetic axis in these self-similar solutions (as $J(\theta) \sim \theta^{2}$ in the in the case of a modest twist, $\Delta \phi_{\mathrm{N}-\mathrm{S}} \lesssim 1$ ).

Many further properties of these solutions are described in Lyutikov \& Thompson (2001).

\subsection{Effects of Slow Rotation}

We have constructed self-similar solutions to the force-free equation in the infinite volume outside a nonrotating spherical surface. A real neutron star rotates, and its rigidly co-rotating magnetosphere has a finite extent, $R \sin \theta \leq c P / 2 \pi \equiv R_{\mathrm{lc}}$ (Goldreich \& Julian 1969). Close to this speed of light cylinder, the rotation will itself cause the field lines to be twisted (Michel 1991; Mestel \& Pryce 1992) - but in a different sense than in the static, twisted magnetosphere. Here we discuss some basic effects of slow rotation, corresponding to an angular velocity of rotation $\Omega \ll c / R_{\mathrm{NS}}$.

The rotational sweeping of the magnetic field lines induces an electric field

$$
\mathbf{E}=-\frac{1}{c}(\boldsymbol{\Omega} \times \mathbf{R}) \times \mathbf{B}
$$

as measured in a background inertial frame. Here $\Omega$ is the angular velocity of the star. The component of $\mathbf{E}$ parallel to $\mathbf{B}$ is cancelled if the closed field lines support a net charge density

$$
\rho=\frac{1}{4 \pi} \nabla \cdot \mathbf{E}=\frac{1}{4 \pi c} \mathbf{\Omega} \cdot[-2 \mathbf{B}+\mathbf{R} \times(\nabla \times \mathbf{B})]=\rho_{\mathrm{GJ}}+\rho_{\text {twist }} .
$$

A second term

$$
\rho_{\text {twist }}=\frac{1}{4 \pi c} \boldsymbol{\Omega} \cdot[\mathbf{R} \times(\nabla \times \mathbf{B})] \simeq \frac{1}{c^{2}} \boldsymbol{\Omega} \cdot(\mathbf{R} \times \mathbf{J})
$$

is now present, as compared with the analysis of Goldreich \& Julian (1969). (The second equality in this expression applies to field lines which close well inside the light cylinder.) Thus, a current flowing in a rotating magnetosphere generates a charge density

$$
\frac{\rho_{\text {twist }}}{J / c} \sim \frac{R}{R_{\mathrm{lc}}},
$$

near the magnetic equator, decreasing to

$$
\frac{\rho_{\text {twist }}}{J / c} \sim\left(\frac{R}{R_{\mathrm{lc}}}\right)^{5 / 2}
$$

on the last closed field lines. 
The term (14) is easily understood to result from the Lorentz boost of the current $\mathbf{J}$ by the rotational velocity $\Omega \times \mathbf{R}$ of the corotating magnetosphere. In radio pulsar models, it has previously been considered only with respect to the open field lines which may carry a space-charge limited current from the stellar surface (Mestel 1993).

The density of charge carriers needed to support a static twist generally exceeds the standard corotation charge density. At radius $R$ and magnetic co-latitude $\theta$, one has $]$

$$
\left|\frac{J / c}{\rho_{\mathrm{GJ}}}\right| \sim \theta^{2}\left(\frac{R}{R_{\mathrm{lc}}}\right)^{-1}\left(\frac{B_{\phi}}{B_{\theta}}\right)_{\theta=\pi / 2} .
$$

Polarization of the positive and negative charges flowing along the magnetic field can therefore supply a corotation charge density of either sign. It is also easy to integrate the current flowing along field lines which extend out to a radius $R$; normalizing to the Goldreich-Julian current $I_{\mathrm{GJ}}=\Omega^{2} R_{\mathrm{NS}}^{3} B_{\text {pole }} / 2 c$, one has

$$
\frac{I(R)}{I_{\mathrm{GJ}}} \simeq\left(\frac{R}{R_{\mathrm{lc}}}\right)^{-2}\left(\frac{B_{\phi}}{B_{\theta}}\right)_{\theta=\pi / 2} .
$$

\subsection{Matching of the Twisted Magnetosphere onto Open Field Lines}

On the open field lines, the corotation charge density could be supplied by a space-charge limited flow of ions and electrons from the surface of the neutron star (e.g. Scharlemann, Arons, \& Fawley 1978). In the absence of electron-positron pairs, one necessarily has $J \simeq \rho c$ in such a flow. Because $\rho_{\mathrm{GJ}}=-\boldsymbol{\Omega} \cdot \mathbf{B} / 2 \pi c$ has the same sign above both magnetic poles, a space-charge limited flow with $\rho=\rho_{\mathrm{GJ}}$ implies a current with an opposing sign in the two magnetic hemispheres. By contrast, the closed loops of current flowing through a twisted magnetosphere will maintain a uniform sign near the magnetic axis.

In the absence of pair creation, such a space-charge limited flow would generate, in one hemisphere and close to the magnetic axis, a toroidal magnetic field with the opposite sign to the more global toroidal field. Figure 5 depicts the distribution of toroidal magnetic field in that hemisphere, assuming a geometry for the current flow that is familar from radio pulsar models: the return current (with a sign opposite to the space-charge limited flow) fills a cylindrical sheath near the boundary between open and closed field lines. This return current forces $B_{\phi} \rightarrow 0$ on some cylindrical surface, outside of which $B_{\phi}$ reverses sign due to the global twist (eq. (18)).

\footnotetext{
${ }^{7}$ We neglect the change in radial index of the field due to the twist.
} 
Pair creation in the space-charge limited flow allows an entirely different relation between charge and current densities $\rho$ and $J$, because the charges of opposite sign can drift with different speeds:

$$
\rho=e\left(n_{+}-n_{-}\right) \simeq \rho_{\mathrm{GJ}}
$$

whereas

$$
J=e\left(n_{+} v_{+}-n_{-} v_{-}\right) \neq \rho_{\mathrm{GJ}} c .
$$

The total number of charge carriers (of either sign) is greatly amplified, by a factor $N_{\text {pair }} \gg 1$, which we leave as a free parameter. The net current becomes

$$
J \simeq \rho_{\mathrm{GJ}} c+N_{\text {pair }}\left|\rho_{\mathrm{GJ}}\right|\left(v_{+}-v_{-}\right)
$$

As a result, a small electric field parallel to $\mathbf{B}$ is sufficient to reverse the sign of $J$ by inducing a small difference in the speed of positive and negative charges $\left|v_{+}-v_{-}\right| / c \sim N_{\text {pair }}^{-1}$. This allows the current to flow in the same direction on the open field lines as the surrounding closed-field current (and in the opposite direction to the original space-charge limited flow). The difference in Lorentz factors $\left|\Gamma_{+}-\Gamma_{-}\right| / \Gamma \sim N_{\text {pair }}^{-1}$ corresponds to a very modest electrostatic potential drop along the outer magnetic field, $\left|e E_{\|} R\right| \sim\left(\Gamma / N_{\text {pair }}\right) m_{e} c^{2}$. Such a 'reversed' current must be intrinsically time-dependent if the seed charges originate on the surface of the neutron star: otherwise, there will be a build-up of charge above the corotation charge density $\rho_{\mathrm{GJ}}$ in the region where pairs are created.

The implications of this effect for the observed (narrow) distribution of spin periods in the SGR and AXP sources are discussed in \$6.1. Fast reconnection of these opposing toroidal fields would force a relaxation of the static twist inside the speed of light cylinder, and thus cause a relaxation in the spindown torque.

\section{Resonant Cyclotron Scattering}

A remarkable feature of these twisted, force-free equilibria is that the current currying charges also provide a significant optical depth to resonant cyclotron scattering. A particle of charge $Z e$ and mass $M$ has a resonance frequency $\omega_{c}=Z e B / M c$, and scatters a photon of frequency $\omega$ (incident at angle $\theta_{k B}$ to B) with cross section $\sigma_{\text {res }}(\omega)=\pi^{2}\left(Z^{2} e^{2} / M c\right) \delta\left(\omega-\omega_{c}\right)\left(1+\cos ^{2} \theta_{k B}\right)$ (e.g. Canuto, Lodenquai, \& Ruderman 1971). In a dipole field, the resonance sits at a radius

$$
r_{\text {res }}=\left(\frac{Z e B_{\text {pole }}}{M c \omega}\right)^{1 / 3} f(\theta)=10.5 Z^{1 / 3}\left(\frac{B_{\text {pole }}}{10^{14} \mathrm{G}}\right)^{1 / 3}\left(\frac{M}{m_{e}}\right)^{-1 / 3}\left(\frac{\hbar \omega}{\mathrm{keV}}\right)^{-1 / 3} f(\theta),
$$


in the approximation of static charges. The angular function $f(\theta)=\left(1-\frac{3}{4} \sin ^{2} \theta\right)^{1 / 6}$. The importance of resonant scattering may be characterized by the resonant optical depth $\tau_{\text {res }}$ :

$$
\tau_{\text {res }}(\omega, \theta)=\int n_{Z}(R) \sigma_{\text {res }}\left(\omega-\omega_{c}(R, \theta)\right) d R=\pi^{2} n_{Z} Z e\left(1+\cos ^{2} \theta_{k B}\right) \frac{R_{\text {res }}}{B\left(R_{\text {res }}\right)}\left|\frac{d \ln R}{d \ln B}\right|,
$$

where $n_{Z}$ is the plasma density at the location of the resonance. The shape of the resonant surface becomes more spherical in the presence of a net twist, but only slightly when $\Delta \phi_{\mathrm{N}-\mathrm{S}} \lesssim 1$ (Lyutikov \& Thompson 2001).

The plasma density at the resonance may be estimated using the current density of the self-similar magnetospheric models considered in Section 3 (eqs. (11) and (田), $J=(c / 4 \pi)\left(B / R_{\mathrm{NS}}\right)[C(p+1) / p]^{1 / 2}\left(\mathcal{P} / \mathcal{P}_{0}\right)^{1 / p}$. Assuming a drift velocity $v^{Z} \leq c$, the particles of charge $Z e$ and mass $M$ generate an optical depth satisfying

$$
\begin{aligned}
\left(\frac{v^{Z}}{c}\right) \tau_{\text {res }} & =\varepsilon^{Z} \frac{\pi}{4}\left(1+\cos ^{2} \theta_{k B}\right)\left[\frac{C(1+p)}{p}\right]^{1 / 2} \frac{[F(\theta)]^{1 / p}}{2+p} \\
& =\frac{\pi \varepsilon^{Z}}{4}\left(1+\cos ^{2} \theta_{k B}\right)\left(\frac{p+1}{p+2}\right)\left[\frac{F(\theta)}{F(\pi / 2)}\right]^{1 / p}\left(\frac{B_{\phi}}{B_{\theta}}\right)_{\theta=\pi / 2}
\end{aligned}
$$

(assuming that the particles carry a fraction $\varepsilon^{Z}$ of the current). Resonant scattering (at much lower frequencies) by a Goldreich-Julian current flowing along open magnetic field lines in a dipolar magnetic field has been considered previously by Rajagopal \& Romani (1997).

Two features of the expression (24) deserve to be emphasized. First, the product $\left(v^{Z} / c\right) \tau_{\text {res }}$ equilibrates to a value near unity when the twist is significant, $B_{\phi} / B_{\theta} \sim 1$ at the magnetic equator, independent of the mass and charge of the scatterers, the radius, or the resonant frequency (with the obvious proviso that the resonant radius sits outside the star). Second, the optical depth to scattering vanishes near the magnetic axis, where the equilibrium current density is reduced as the result of the large extent of the field lines (Fig. (4). The angular factor in eq. (24) is $[F(\theta)]^{1 / p} \simeq \sin ^{2} \theta$ for twists $\Delta \phi_{\mathrm{N}-\mathrm{S}} \lesssim 1$ radian $\left(F \simeq 1-\mu^{2}, p \simeq 1\right)$.

Thus the emission leaving the surface of the neutron star near equatorial plane will be strongly scattered at the cyclotron resonance, while at the poles the emission will emerged almost unscattered. This axisymmetric, self-similar model will more accurately describe the magnetospheric structure of an SGR/AXP at larger distances from the star, where the magnetic field lines are anchored in a small portion of the neutron star surface, and their arrangement is less sensitive to irregularities in the surface flux density. As a result, the scattering surface should more closely approximate this simple, axisymmetric form at lower frequencies, and at the electron cyclotron resonance than at the ion resonance(s). 
A similar result can be derived in any situation where the magnetic field is strongly sheared, and can be applied to more complicated (e.g. multipolar) magnetic field geometries. The resonant optical depth along a ray is inversely proportional to the gradient of $B$ parallel to the ray,

$$
\tau_{\text {res }}=\pi^{2}(Z e) n_{Z}\left(1+\cos ^{2} \theta_{\mathrm{kB}}\right)\left|\frac{d l}{d B}\right| .
$$

Estimating $(Z e) v^{Z} n^{Z} \sim \frac{1}{2}(c / 4 \pi)|\nabla \times \mathbf{B}|$ (the resonant charges carry half the current), one has

$$
\tau_{\text {res }}\left(\frac{v^{Z}}{c}\right)=\frac{\pi}{8}\left(1+\cos ^{2} \theta_{\mathrm{kB}}\right)|\nabla \times \mathbf{B}|\left|\frac{d l}{d B}\right| .
$$

Strong shearing of the magnetic field corresponds to $|\nabla \times \mathbf{B}||d l / d B| \sim 1$, and implies strong Doppler heating within the scattering layer ( $\$ 5.4$ ).

\subsection{Equilibrium Drift Speed}

Current-carrying charges will feel a drag force when they enter a region with a significant flux of photons at the cyclotron energy. In the quiescent state of an SGR (or AXP) source, the drag force acting on the ion component of the current is only a modest fraction of gravity (Appendix B). The minimum drift speed of the upward moving ions at the surface of the star can then be related to the gravitational potential difference which they traverse along a magnetic flux line,

$$
\Delta \phi=\left(1-\frac{R_{\mathrm{NS}}}{R_{\max }}\right) \frac{G M_{\mathrm{NS}}}{R_{\mathrm{NS}}},
$$

through

$$
\frac{v_{i}(\min )}{c}=\left(\frac{2 \Delta \phi}{c^{2}}\right)^{1 / 2} \simeq 0.6\left(1-\frac{R_{\mathrm{NS}}}{R_{\max }}\right)^{1 / 2} .
$$

The drift speed will decrease as the ions climb the potential.

The drag force acting on the electrons is largest at a radius $R \sim 100 \mathrm{~km}$, where their cyclotron energy is in the keV range. The spectral energy density of the radiation field can then be estimated as $U_{\omega} \sim L_{\omega} / 4 \pi R^{2} c$. An electron moving along $\mathbf{B}$ with a particular speed $v_{X}$ will feel a Compton drag force which is perpendicular to $\mathbf{B}$, so that the electron does not gain or lose kinetic energy. In general $v_{X}$ is a modest fraction of the speed of light $c$ and does not equal the drift speed $v_{e}$ of the current-carrying electrons: for example, $v_{X}=\cos \theta_{\mathrm{kB}}$ when the photons stream at a fixed angle $\theta_{\mathrm{kB}}$ with respect to $\mathbf{B}$. To clarify the discussion that follows, we consider only the simplest case where the radiation field is directed perpendicular to $\mathbf{B}$, and $v_{X}=0$. 
The component of the Compton draft force parallel to $\mathbf{B}$ can be written as

$$
F_{\|} \sim \Gamma_{e}^{2}\left(\frac{v_{e}}{c}\right) \int \frac{L_{\omega}}{4 \pi R^{2} c} \sigma\left(\omega, \theta_{\mathrm{kB}}=\pi / 2\right) d \omega
$$

here $v_{e}$ and $\Gamma_{e}$ are the speed and Lorentz of the electron along B. Substituting the resonant cross section, one finds that this drag force is easily sufficient to slow an electron to sub-relativistic speed, unless it is compensated by an electrostatic force

$$
e E_{\|} \sim \frac{\pi}{4} \Gamma_{e}^{2}\left(\frac{v_{e}}{c}\right)\left(\frac{e^{2}}{m_{e} c^{2}}\right)\left(\frac{L_{\omega_{c}}}{R_{\mathrm{res}}^{2}}\right) .
$$

The measured spectrum of a magnetar is low enough in the optical bands (Hulleman et al. 2000) that the ions feel a negligible Compton drag force at the same radius that the electrons resonantly scatter off $\mathrm{keV}$ photons. Thus, after exiting the electron resonance layer, the ions (which we take to be protons) will have a minimum kinetic energy

$$
\left(\Gamma_{p}-1\right) m_{p} c^{2} \sim e E_{\|} R_{\mathrm{res}}
$$

Now the assumption of a steady current flow, combined with charge neutrality $\left(n_{p}=n_{e}\right)$, leads to the requirement that the ratio of drift speeds $v_{e} / v_{p}$ maintain a constant value $\varepsilon_{e} / \varepsilon_{p}$ within the resonance scattering layer. (As before, $\varepsilon_{i}$ is the fraction of the current carried by particle species $i$. Notice that only a tiny charge separation $\left|n_{p}-n_{e}\right| / n_{e}=O\left(10^{-12}\right)$ is needed to maintain the electrostatic field (30).) In the simplest case where $v_{e}=v_{p}$, one deduces

$$
\frac{\Gamma_{\mathrm{eq}}-1}{\Gamma_{\mathrm{eq}}^{2}\left(v_{\mathrm{eq}} / c\right)} \sim \frac{\pi e^{2}}{4 m_{p} m_{e} c^{4}} \frac{\left(\omega L_{\omega}\right)_{\omega_{c}}}{R_{\mathrm{res}} \omega_{c}}
$$

for the equilibrium drift speed (Lorentz factor) within the resonant scattering layer. Re-expressing the resonant radius $R_{\text {res }}$ in terms of the surface polar (dipole) field, this equation becomes

$$
\frac{\Gamma_{\mathrm{eq}}-1}{\Gamma_{\mathrm{eq}}^{2}\left(v_{\mathrm{eq}} / c\right)} \sim 0.9\left[\frac{\left(\omega L_{\omega}\right)_{\omega_{c}}}{10^{35} \mathrm{erg} \mathrm{s}^{-1}}\right]\left(\frac{\hbar \omega_{c}}{\mathrm{keV}}\right)^{-2 / 3}\left(\frac{B_{\mathrm{pole}}}{10^{14} \mathrm{G}}\right)^{-1 / 3}\left(\frac{R_{\mathrm{NS}}}{10 \mathrm{~km}}\right)^{-1}
$$

One observes that mildly relativistic motion of the charge carriers is implied if the X-ray luminosity of the source is $\sim 10^{35} \mathrm{erg} \mathrm{s}^{-1}$. The left side of eq. (33) has a maximum value of $\simeq 0.3$ at $v_{\text {eq }} / c \simeq 0.8$. The current must, as a result, be dominated by ions when $L_{X}$ significantly exceeds $\sim 10^{35} \mathrm{erg} \mathrm{s}^{-1}$. 


\section{Implications for X-ray Spectra and Pulse Profiles}

We have described the basic properties (magnetic field geometry, optical depth to resonant scattering) of a class of twisted, self-similar solutions to the force free equation. In this section, we show how several observed properties of the SGR and AXP sources connect directly with this model, which provides a promising framework for synthesizing the two populations.

\subsection{Surface Heating (vs. Volumetric Heating)}

Transport of a magnetic field in the deep crust and core of a magnetar will result in dissipation both inside and outside the star (TD96). The heat released in the core (through frictional heating and beta reactions) is converted largely to neutrinos, and will be conducted to the surface with a delay of one year or longer if the surface temperature is $\sim 0.5 \mathrm{keV}$. Non-potential distortions of the magnetic field outside the star are converted much more efficiently to visible electromagnetic radiation (X-rays), because the associated particle flows are largely confined to closed magnetic field lines. Changes in the external magnetic field will, as a result, induce equally sudden changes in the source brightness (e.g. SGR flares).

In the present context, where we are interested in a smooth shearing deformation of the magnetic field, the dissipation outside the star takes two principal forms: impact of the current-carrying charges on the stellar surface; and resonant Comptonization of this surface X-ray flux by the magnetospheric currents. The surface heating provides a minimal radiative output of a magnetar, which is increased by a modest factor as the X-rays undergo multiple scattering (\$5.4). When $B_{\text {dipole }}$ (multiplied by the irradiated fraction of the stellar surface) is $\sim 10^{14} \mathrm{G}$, we find that the surface X-ray flux is, by coincidence, comparable to the equilibrium flux powered by ambipolar diffusion of a strong internal magnetic field (TD96; Heyl \& Kulkarni 1998). The angular pattern of the surface radiation can be expected to differ significantly from models of passively cooling magnetars; the implications for pulse fractions are discussed in $\$ 5.5$.

Both positive and negative charges are needed to cancel the space charge outside the neutron star. Let us consider the simplest case where the surface has a light element composition, and positive ions (e.g. hydrogen, helium, or carbon) can be emitted thermionically (Thompson et al. 2000). The electric field which lifts an ion through the gravitational potential of the star is many orders of magnitude smaller than the field induced by a bare space charge $\rho \sim J / c$. A first estimate of the minimum power consumed 
by the current is obtained by summing the gravitational potential energy of the ions falling onto the cathode, with the (approximately equal) electrostatic energy picked up by returning electrons at the anode, where the ions are accelerated. The contribution to the power from an infinitesimal current $J\left(R_{\mathrm{NS}}\right) d A$ originating in a surface element $d A$ is then $d L_{X}=2\left(G M_{\mathrm{NS}} m_{p} / R_{\mathrm{NS}}\right)\left(1-R_{\mathrm{NS}} / R_{\max }\right) J\left(R_{\mathrm{NS}}\right) d A / e$. Here $R_{\max } / R_{\mathrm{NS}}=[F(\theta) / F(\pi / 2)]^{-1 / p}$ is the maximum radius attained by a field line anchored at polar angle $\theta$. One observes that the heating rate is strongly inhomogeneous over the surface of the star, even under the assumption of a cylindrically symmetric and self-similar magnetosphere. The current density varies across the stellar surface, as does the gravitational potential shift along magnetic flux lines of different lengths.

Integrating over the surface of the star, we find

$$
\begin{aligned}
L_{X} & =\frac{B_{\text {pole }} G M_{\mathrm{NS}} m_{p} c}{2 e} \frac{p F(\pi / 2)}{2+p}\left(\frac{B_{\phi}}{B_{\theta}}\right)_{\theta=\pi / 2} \\
& \simeq 3 \times 10^{35}\left(\frac{B_{\text {pole }}}{10^{14} \mathrm{G}}\right)\left(\frac{M_{\mathrm{NS}}}{1.4 M_{\odot}}\right)\left(\frac{B_{\phi}}{B_{\theta}}\right)_{\theta=\pi / 2} \quad \mathrm{erg} \mathrm{s}^{-1} .
\end{aligned}
$$

In this self-similar model, the rate of surface heating is close to the persistent X-ray luminosity of the SGR and AXP sources if $B_{\text {pole }} \times\left(B_{\phi} / B_{\theta}\right)_{\theta=\pi / 2} \sim 10^{14} \mathrm{G}$. The heating rate is too high if the magnetosphere is strongly twisted and $B_{\text {pole }} \sim 10^{15} \mathrm{G}$, but in reality the twist could be more localized - e.g. around the magnetic poles.

There is an inefficiency in driving this form of dissipation by ambipolar diffusion of the core magnetic field: the total radiative output of an AXP or SGR is $\sim 10^{47}$ ergs over its $\sim 10^{4}$ year lifetime, but the total energy radiated in neutrinos is some $\sim 30$ times higher. The minimal (r.m.s.) magnetic field needed to power the observed activity is $\sim 10^{15} \mathrm{G}$, which increases by a factor $\sim 5$ to allow rapid diffusion of the field through the core. In $^{\text {In }}$ this regard, the Sun provides a rough analog: distortions of the magnetic field outside its photosphere are converted efficiently to non-thermal radiation (Solar flares). However, only a tiny fraction of the total energy stored in the Solar magnetic field is actually released in its exterior: only one part in a thousand of the bolometric Solar output is radiated through the chromosphere and corona. In a magnetar, motions of the external magnetic footpoints are driven gradually by diffusive processes in the deep crust and core, and

\footnotetext{
${ }^{8}$ These fields are easily strong enough to break the neutron star's rigid crust; but observations of magnetic white dwarfs demonstrate the existence of stable magnetostatic equilibria even in perfectly fluid, degenerate stars. When the magnetic field is pinned by a rigid crust, transport of the field will force the build-up of shear stresses in the crust to a (small) fraction of the bulk magnetostatic stresses; see Thompson \& Duncan (1995), (2001).
} 
also suddenly by brittle fractures of its crust. We focus in this paper on smooth shear deformations of the external magnetic field, but the possibility remains that a magnetar will also experience an approximate analog of 'microflaring' on the Sun: the Hall electric field will drive high-frequency dislocations of its crust $(\lambda \lesssim 0.1 \mathrm{~km})$, which couple efficiently to magnetospheric Alfvén waves (TD96).

\subsection{Decay of the External Twist}

The preceding result (eq. [34]) also allows a simple estimate of the maximum time for the external twist to dissipate completely, in the absence of further sub-surface motions. The energy of a twisted magnetosphere exceeds the energy of a pure dipole with the same polar flux density,

$$
\frac{E_{B}}{E_{B}(\text { dipole })}=\frac{3}{2(p+1)} \int_{0}^{1} d \mu\left(\frac{d F}{d \mu}\right)^{2}>1
$$

and can be approximated by

$$
\frac{E_{B}}{E_{B}(\text { dipole })}=1+0.17 \Delta \phi_{\mathrm{N}-\mathrm{S}}^{2}
$$

for $\Delta \phi_{\mathrm{N}-\mathrm{S}} \lesssim 1$. This works out to

$$
E_{B}-E_{B}(\text { dipole })=1.4 \times 10^{44} \Delta \phi_{\mathrm{N}-\mathrm{S}}^{2}\left(\frac{B_{\text {pole }}}{10^{14} \mathrm{G}}\right)^{2}\left(\frac{R_{\mathrm{NS}}}{10 \mathrm{~km}}\right)^{3} \quad \mathrm{erg},
$$

for twists $\lesssim 1$ radian. The decay time for the global twist is then

$$
t_{\text {decay }}=\frac{E_{B}-E_{B}(\text { dipole })}{L_{X}}=40 \Delta \phi_{\mathrm{N}-\mathrm{S}}^{2}\left(\frac{L_{X}}{10^{35} \mathrm{erg} \mathrm{s}^{-1}}\right)^{-1}\left(\frac{B_{\text {pole }}}{10^{14} \mathrm{G}}\right)^{2}\left(\frac{R_{\mathrm{NS}}}{10 \mathrm{~km}}\right)^{3} \quad \mathrm{yr} .
$$

very similar to the estimate made in Thompson et al. (2000).

The amount of surface heating would be reduced by a factor $\gamma_{e}\left(m_{e} / m_{p}\right)\left(c^{2} / G M_{\mathrm{NS}}\right)-$ and the lifetime of the external twist would be increased correspondingly - if the current were carried by relativistic pairs of energy $\gamma_{e} m_{e} c^{2}$.

\subsection{The Ion Cyclotron Resonance and Higher Multipoles}

In this self-similar model, the ion component of the current will generate a comparable optical depth to the electron component in a self-similar magnetosphere. The optical depth 
to resonant scattering is independent of the net mass or electrical charge, excepting the factor $\varepsilon^{Z}\left(v^{Z} / c\right)^{-1}$ (eq. 24.) Indeed, the cyclotron energy of an ion of charge $+Z e$ and mass $A m_{n}$ in a magnetic field $B$ is $\hbar \omega=6.3(Z / A)\left(B / 10^{15} \mathrm{G}\right) \mathrm{keV}$. Although this resonance sits at X-ray frequencies in magnetar-strength fields, the resonant radius (22) is $\sim 10$ times smaller for ions than electrons (or positrons).

The heavier particles will resonantly scatter a more restricted range of frequencies (below their surface cyclotron frequency). This means that higher magnetic multipoles will leave a more direct imprint in the pulse profile through resonant ion scattering, than electron scattering. Notice also that higher multipoles will increase the surface field strength over the polar dipole value, and hence increase the range of frequencies which resonantly scatter close to the star. The pulse profile of the August 27 giant flare provides direct evidence for the presence of higher magnetic multipoles in SGR 1900+14: four sub-pulses of a large amplitude appeared during the intermediate portion of the burst, which repeated coherently with the 5.16 spin period (Feroci et al. 2001; Thompson \& Duncan 2001).

\subsubsection{Surface Stopping of Magnetospheric Charges and Ion Cyclotron Emission}

The surface of a magnetar will be impacted by ions or electrons, depending on the sign of the electrical current. The characteristic kinetic energy of an ion is its gravitational binding energy to the star, $E_{i} \sim G M_{\mathrm{NS}}\left(A m_{p}\right) / R_{\mathrm{NS}}=200 A \mathrm{MeV}$. If the ion flow contains a significant proton component, then a layer of hydrogen will form at the cathode surface. The downward-moving protons will be stopped by $p-p$ collisions at a depth corresponding to an electron column $\sigma_{T} N_{e} \simeq 30$.

We point out here the interesting possibility that ion cyclotron emission line may be observed in magnetars whose surfaces are heated by magnetospheric charges. The absorption feature generated near the ion cyclotron resonance of a passively cooling magnetar has been investigated recently by Zane et al. (2001) and Ho and Lai (2001). At the cathode, a significant fraction of the energy of the impacting charges is transferred directly to the ions. This spectral feature is easiest to detect if the surface magnetic field lies in the range $B_{S}=(0.2-2) \times 10^{15}(A / Z) \mathrm{G}$, so that the ion cyclotron resonance sits between 1 and $10 \mathrm{keV}$. Phase-resolved spectroscopy of magnetar candidates could, for this reason, provide a direct measure of the fraction of the thermal emission powered by magnetospheric currents.

\footnotetext{
${ }^{9}$ Resonant absorption of cyclotron photons will, during a bright X-ray phase, exert a stronger force than gravity (Ibrahim et al. 2001; Appendix B.1).
} 
The nature of the radiative transport near the ion cyclotron resonance is substantially different in 'naked' magnetars with persistent magnetospheric currents, than in accreting neutron stars. Below a critical radiative flux $\omega_{c, i} F_{\omega_{c, i}} \sim(J / e)(A / Z) G M_{\mathrm{NS}} m_{p} / R_{\mathrm{NS}}$ (eq. B3), the density of returning, current-carrying ions is almost constant above the surface of a magnetar. This stands in contrast with an accreting neutron star whose surface electron cyclotron frequency sits in the X-ray range, close to which a strong radiative force on the accreting electrons will allow the infalling matter to settle gradually. The resonant optical depth (eq. 23]) is proportional to the local density of scattering charges (rather than to the column density as with non-resonant scattering). This means that $\tau_{\text {res }}$ has a strong spike just above the surface of an accreting neutron star, but remains approximately constant above the surface of an isolated magnetar with a non-potential magnetic field. In the accreting case, the result is the formation of an absorption feature at an X-ray cyclotron resonance (Zane, Turolla, \& Treves 2000). In the magnetar case, by contrast, the kinetic energy lost by the impacting charges will raise the temperature in a thin surface layer without a large cyclotron opacity developing outside that layer. This allows an emission feature to form at a cyclotron resonance. (Nelson et al. 1995 have demonstrated this effect for electron cyclotron scattering in a $\sim 10^{12} \mathrm{G}$ magnetic field, at the surface of a neutron star which is impacted by ions moving at the free fall speed.)

\subsection{Resonant Compton Heating}

Multiple scattering at the cyclotron resonance will redistribute photons in frequency. This effect has some similarities with Comptonization in an accretion flow (Blandford \& Payne 1981), except that resonant scattering of a photon of a given frequency occurs only at a particular resonant surface: space and energy diffusion are now directly coupled. Photons which are backscattered return to a region where their energy lies below the local cyclotron resonance, and will undergo subsequent resonant scatterings.

The optical depth to resonant scattering by charges moving with drift speed $v$ is $\simeq(v / c)^{-1}\left(B_{\phi} / B_{\theta}\right)_{\theta=\pi / 2}$ at the magnetic equator (eq. 24]). Let us first consider the case of electron scattering. Resonant scattering by $\sim \mathrm{keV}$ photons at a radius $R / R_{\mathrm{NS}} \sim 10\left(B_{\text {pole }} / 10^{14} \mathrm{G}\right)^{1 / 3}$ maintains a mildly relativistic electron drift speed when $\omega_{c} L_{\omega_{c}}=10^{35} \mathrm{erg} \mathrm{s}^{-1}(\S 4.1)$. In addition, the product $\left(v_{e} / c\right) \tau_{\text {res }}$ has no explicit frequency dependence below an energy $\hbar \omega \sim m_{e} c^{2}$, and above the very low cyclotron frequency at the speed of light cylinder $\omega_{c, e} / 2 \pi \sim 10^{8} \mathrm{~Hz}$.

In spite of the complicated geometry of the magnetosphere, we can draw a remarkably simple conclusion: the product of the mean frequency shift per scattering $\Delta \omega / \omega$ and the 
number of scatterings is $O(1)$ when the twist angle $\Delta \phi_{N-S} \sim 1$. As a result, multiple resonant scattering of thermal X-ray photons, within a self-similar twisted magnetosphere, provides a simple mechanism for generating a high energy tail to the X-ray spectrum. In the case of electron cyclotron scattering, this high energy tail will extend up to a frequency $\hbar \omega_{\max } \sim \frac{1}{2} m_{e} v_{e}^{2} \gtrsim 100 \mathrm{keV}$, where the increase in frequency due to the Doppler effect is balanced by the Compton recoil.

At a fixed spectral intensity, the hardness of the high energy tail will increase with the number of scatterings and, hence, with the net twist imparted to the external magnetic field. However, this relation between spectral hardness and magnetospheric current can be compensated by changes in spectral intensity, for the reason that the drift speed of the electrons varies as a complicated function of $L_{\omega_{c}}$ when $\left(\omega L_{\omega}\right)_{\omega_{c}} \lesssim 10^{35} \mathrm{erg} \mathrm{s}^{-1}$ (\$.1). The often measured ratio of power-law to thermal intensities should also be interpreted with caution in this situation: if the thermal seed is generated primarily by surface heating, then its spectrum will not be a pure black body, and a spectral decomposition into black body and power-law components will not accurately measure the relative luminosities carried by the surface and magnetospheric components.

The particles which support the magnetospheric current will, on balance, transfer energy to the photons. This statement must be qualified if, however, the photon flux at the surface of the star is high enough to drive a wind (Ibrahim et al. 2001; Appendix B.1). In that case, a cloud of particles with $\tau_{\text {res }} \gtrsim 1$ will be suspended in the magnetosphere predominantly by radiation forces. Even in the absence of an electrical current, the kinetic energy of a suspended particle can, in principle, significantly exceed the energy of a seed photon: for example, if a photon bubble instability drives buoyant oscillations of the particles with respect to the photons (Arons 1992).

\subsubsection{Scattering at the Ion Cyclotron Resonance}

The optical depths to ion and electron cyclotron scattering are comparable in this self-similar model. More generally, if the surface magnetic field is strongly sheared, then a the product of the resonant optical depth and the ion drift speed satisfies $\tau_{\text {res }}\left(v^{Z} / c\right) \sim 1$ across a resonant layer (§田).

However, multiple scattering by ions is different in three important respects. First, as discussed in Section 5.3, it is sensitive to the presence of higher-order multipoles in the magnetic field. Second, the upper cutoff to the non-thermal spectral tail is not set by the 
Compton recoil, but instead by the surface cyclotron frequency,

$$
\hbar \omega_{\max }=\frac{\hbar \omega_{c, i}\left(R_{\mathrm{NS}}\right)}{\gamma_{i}\left(1-v_{i} / c\right)} \simeq \frac{30}{\gamma_{i}\left(1-v_{i} / c\right)}\left(\frac{Z}{A}\right)\left(\frac{B}{100 B_{\mathrm{QED}}}\right) \quad \mathrm{keV} .
$$

In this expression, the Doppler factor $\gamma_{i}^{-1}\left(1-v_{i} / c\right)^{-1}$ takes into account the (upward) motion of the ions with speed $v_{i}$. Even if the ions are moving sub-relativistically, their kinetic energy $\frac{1}{2}\left(A m_{p}\right) v_{i}^{2}$ will greatly exceed $\hbar \omega_{c, i}$. A non-thermal spectrum extending above $20 \mathrm{keV}$ can be produced by resonant ion scattering if the surface field is stronger than $\sim 3 \times 10^{15} \mathrm{G}$, i.e., if higher multipoles are present in the magnetic field. Indeed, higher multipoles are almost certainly present in the SGR sources (Feroci et al. 2001).

Third, a photon has a much larger probability of being backscattered directly at the neutron star within the ion corona $(R \sim 10-20 \mathrm{~km})$ than in the electron corona $(R \sim 50-100 \mathrm{~km})$. The simplest geometry involves an ion current converging toward the surface of the star, where photons are reflected upward by (non-resonant) electron scattering if they are in the ordinary polarization mode. Repeated scatterings between the converging ion current and the surface results in first order Fermi acceleration of a photon to higher energy. Multiple ion scattering can also occur entirely within the magnetosphere, and is most effective at generating a high energy spectral tail if ions of different charge/mass ratios $Z / A$ are present, or if ions flow both toward and away from the surface of the star at neighboring locations. The heavier ions, having a lower charge/mass ratio $Z / A \simeq \frac{1}{2}$, will resonantly scatter X-rays closer to the star. This means that the resonance condition $\omega=\omega_{c, i} / \gamma_{i}\left(1 \mp v_{i} / c\right)$ can be satisfied for both outward-moving and inward-moving photons if the backscattering is by the protons at a larger radius. The inner and outer resonant surfaces are converging in a region where the ions are streaming outward from the star (and losing kinetic energy in its gravitational potential), and an extended high-energy spectral tail will result.

\subsubsection{Non-resonant Electron Scattering.}

The (angle-averaged) electron scattering cross section of one photon polarization mode (the O-mode) is close to Thomson, even at frequencies well below the first electron Landau resonance. It is therefore worthwhile noting that the Thomson scattering depth through a current carrying magnetic field can approach unity if the flux density exceeds $\sim 100 B_{\mathrm{QED}}$, and the field is strongly sheared. We estimate $n_{e} v_{e} \sim J / 2 e=(c / 8 \pi e)|\nabla \times \mathbf{B}|$ for an electron-ion current. One then finds

$$
\tau_{T} \simeq n_{e} \sigma_{T} \ell=\frac{1}{3} \alpha_{\mathrm{em}}\left(\frac{B}{B_{\mathrm{QED}}}\right)\left(\frac{v_{e}}{c}\right)^{-1}
$$




$$
=0.24\left(\frac{B}{100 B_{\mathrm{QED}}}\right)\left(\frac{v_{e}}{c}\right)^{-1}
$$

in the case of strong shear (size $\ell$ of the current-carrying region comparable to $B /|\nabla \times \mathbf{B}|)$. The optical depth increases by a factor $\sim 2$ when the current is supported by electron-positron pairs.

When pairs are the dominant charge-carriers, the mean frequency shift of a photon is second order in the drift speed of the charges and, in the optically thick limit $\tau_{T} \gg 1$, each photon scatters $\sim \tau_{T}^{2}$ times before escaping the 'pair corona'. In this case, the Compton parameter depends only on the amount of shear and on the strength of the field (Thompson \& Duncan 2001). One has

$$
y \simeq \frac{4}{3} \tau_{T}^{2}\left(\frac{v_{e}}{c}\right)^{2} \simeq \frac{16}{27}\left(\alpha_{\mathrm{em}} \frac{B}{B_{\mathrm{QED}}}\right)^{2}
$$

for a strongly sheared field. A high density of pairs can be self-consistently maintained by $\gamma-\gamma$ collisions during an SGR burst, when the plasma temperature exceeds $\sim 30 \mathrm{keV}$ (Thompson and Duncan 1995, 2001). A self-consistent pair corona is also possible at much lower luminosities, through multiple Compton upscattering of seed X-rays into an extended non-thermal spectral tail. This requires $y \gtrsim 1$, corresponding to static magnetic fields stronger than $B \sim 200 B_{\mathrm{QED}} \sim 10^{16} \mathrm{G}$ (eq. 41]).

\subsubsection{Comparison with the SGR and AXP Sources.}

The spectra of some AXPs are inferred to have very soft power-law components (photon index $\sim-4$ ). These soft spectra may be explainable in terms of passive radiative transport through the surface of a neutron star with a $\sim 10^{14} \mathrm{G}$ magnetic field (Özel 2001; Ho \& Lai 2001; Lai \& Ho 2001). It should, nonetheless, be kept in mind that the spectrum of one AXP is much harder (the photon index is -2.5 for 1E 1048.1-5937; Oosterbroek et al. 1998); and the actively bursting SGRs have even harder spectra (photon indices -2.2; Hurley 2000, and references therein). Multiple resonant scattering provides a mechanism for generating the full observed range of power-law indices: the softest AXP spectra correspond to external magnetic fields which carry relatively weak electrical currents, and the hardest SGR spectra to magnetospheres which are strongly twisted.

It is interesting to note that $t_{\text {decay }}$ (eq. [38]) is comparable to the time since the 5 March 
1979 giant flare if the polar field $\square$ is $\simeq 10^{14} \mathrm{G}$. The relatively soft power-law spectrum of SGR 0526-66 (Kulkarni et al. 2001) may therefore have an explanation in terms of the decay of the external magnetic shear following the flare.

As we have discussed, the atmosphere of a magnetar can be expected to have more than one scattering corona. The observations do not yet allow us to distinguish between the case where the coronal heating is dominated by electron cyclotron scattering, versus ion cyclotron scattering (or non-resonant $e^{ \pm}$scattering) closer to the star. Only scattering by electrons can create a non-thermal spectrum extending up to $\sim m_{e} c^{2}$ if the charges are mildly relativistic (\$4.1). For this reason, high energy observations of persistent SGR spectra (between 20 and $500 \mathrm{keV}$ ) would provide a clear discriminant between coronal heating dominated by electron vs. ion scattering.

More detailed calculations of the distribution of scatterings and frequency shifts are under way.

\subsection{Pulsations in the Persistent Emission}

The Anomalous X-ray Pulsars have been proposed to be either magnetars powered by active field decay (TD96), or passively cooling neutron stars with $\sim 10^{14} \mathrm{G}$ magnetic fields (Heyl \& Hernquist 1997). In addition to the constancy of the measured X-ray flux, these models may also be distinguished by the pattern of the emergent X-ray pulsations. The X-ray spectra and pulse profiles produced by passively cooling neutron stars have been calculated by Psaltis, Özel, \& DeDeo (2000), Özel (2001), and Özel, Psaltis, \& Kaspi (2001), taking into account the gravitational deflection of the photons, the angular variation of the opacity, and the interchange between the two X-ray polarization modes in the outer atmosphere. They find that the pulse fractions are less than those measured in two AXPs (e.g. Table 2 in Kaspi et al. 2001), even in the case of cooling through a single, localized hotspot. 1 Nonetheless, as we now describe, bombardment of the neutron star surface by persistent currents, and re-scattering of the X-rays in the magnetosphere, can lead to significantly different pulse profiles.

It should first be emphasized that the angular distribution of the surface X-ray flux depends on the relative proportions of the flux carried by the extraordinary polarization

\footnotetext{
${ }^{10}$ To be more precise, the dipole field in the absence of any external twist.

${ }^{11}$ As emphasized by Özel et al. (2001), this conclusion is dependent on systematic bias in the distances to these sources.
} 
mode (the E-mode) and the ordinary mode (the O-mode). The O-mode radiation is strongly beamed at the surface of a neutron star, because its scattering opacity scales as ${ }^{12} \sin ^{2} \theta_{k B}$ at frequencies well below the electron cyclotron frequency (Basko \& Sunyaev 1975). However, a large fraction of the energy flux across the surface of a passively cooling neutron star is carried by the E-mode, whose opacity is more isotropic.

At the surface of a magnetar, the beaming of the O-mode radiation is most important when the X-ray flux is generated by ion heating. In that case, most of the surface flux will emerge in the O-mode and, in addition, the heating may be localized at a small hot spot, which allows the formation of a narrow cone of radiation. The opacity of the E-mode is suppressed in an ultrastrong magnetic field by a factor $\left(m_{e} c \omega / e B\right)^{2}=7 \times 10^{-7}(\hbar \omega / \mathrm{keV})^{2}\left(B / 10^{14} \mathrm{G}\right)^{-2}$. Impacting protons will be stopped by $p-p$ collisions at a depth corresponding to $N_{e} \sigma_{T} \sim 30$ - well above the position of the E-mode photosphere but, generally, below the O-mode photosphere.

\subsubsection{Cyclotron Scattering in the Magnetosphere}

Resonant cyclotron scattering will strongly modify the angular pattern of the X-ray flux emerging from a twisted, current-carrying magnetosphere. This reprocessing will occur at the electron cyclotron resonance at a large distance of $\sim 50-100 \mathrm{~km}$, where gravitational bending of the photon trajectories can be largely neglected. Reprocessing will also occur at the ion cyclotron resonance closer to the star, at $10-20 \mathrm{~km}$.

Three effects are important here:

1. The optical depth to resonant scattering is a strong function of angle in a self-similar, twisted magnetosphere (Fig. 凹). Even when the twist is large, $\Delta \phi_{\mathrm{N}-\mathrm{S}} \sim 1$ radian, and the optical depth exceeds unity at the magnetic equator, the resonant scattering surface maintains two holes of solid angle $\sim 2 \mathrm{Sr}$, centered at the two magnetic poles. This effect will increase the relative X-ray flux emerging along the magnetic axes.

2. The scattered cyclotron radiation is beamed parallel to $\mathbf{B}: d \sigma_{\text {res }} / d \Omega \propto 1+\cos ^{2} \theta$ in the rest frame of the scattering charge. A signature of this effect is the appearance of a fourth-order harmonic in the pulse profile. Note also that the degree of beaming can be greatly enhanced by the bulk motion of the charge carriers in an electron-ion current. Consider, for example, an optically thick scattering screen which moves at uniform speed $v$ along the magnetic field. The intensity of the radiation emerging from the screen is

\footnotetext{
${ }^{12}$ As before, $\theta_{k b}$ is the angle between the background magnetic field and the photon wavevector.
} 
concentrated at an angle $\theta=\cos ^{-1}(v / c)$ with respect to $\mathbf{B}$.

3. The resonant surface is aspherical, with a cross-sectional area that is larger when viewed along the magnetic equator, than from one of the magnetic poles. This effect will enhance the relative flux propagating to infinity along the magnetic equator.

Each of these effects can, by themselves, generate one or two sub-pulses - depending on the orientation of the rotation and magnetic axes with respect to each other and to the line of sight. Calculations of the resultant pulse profiles are underway.

Even if the magnetic field were axisymmetric in the absence of electrical currents, deformations of the neutron star crust which generate the currents could have azimuthal structure. Resonant scattering by non-axisymmetric currents therefore provides an additional source of structure in the X-ray pulse profile.

\subsection{Implications for Giant Flare Mechanism}

There are two generic possibilities for the production of the giant flares of the SGRs, in the framework of our model (Thompson \& Duncan 1995, 2001; Woods et al. 2001a). First, a giant flare may result from a sudden change (unwinding) in the internal magnetic field. In this case, a twist is implanted into the magnetosphere. A large-scale displacement of the crust probably requires the formation of a propagating fracture, close to which the magnetic field is strongly sheared. An attractive mechanism for powering the flare involves the repeated excitation and relaxation of a high current density around the fault. The energy stored in a twisted internal field, which is available for sudden release, can be related to the limiting strain $\psi_{\max }$ of the crust, ${ }^{3} \Delta E_{B}=1 \times 10^{46}\left(\psi_{\max } / 0.01\right)^{2}\left(B_{\text {pole }} / 10^{14} \mathrm{G}\right)^{-2} \mathrm{erg}$ (Thompson \& Duncan 2001). In the aftermath of the flare, the magnetic field will retain a more smoothly distributed component of the shear. According to this first scenario, the X-ray spectrum should be harder when the X-ray flux has returned to the pre-burst value, but the pulse profile may be simpler as the result of multiple cyclotron scattering.

Alternatively, giant flares may involve a sudden relaxation in the twist outside the star, without the impetus of sudden subsurface motions, in close analogy with Solar flares. This requires that the external magnetic shear build up gradually, and that the outer crust of the neutron star is deformed plastically by internal magnetic stresses. This mechanism

\footnotetext{
${ }^{13}$ Here we have normalized $B_{\text {pole }}$ to the minimum value needed to power the giant flares through shearing and reconnection of an external magnetic field, triggered by subsurface motions (Thompson and Duncan 2001).
} 
has the advantage that the energy stored in the external twist need not be limited by the tensile strength of the crust, but instead by the total external magnetic field energy. From eq. (37), one infers that a polar dipole field of $\sim 3 \times 10^{14} \mathrm{G}$ is needed to power a flare of energy $\sim 10^{45}$ erg through the sudden relaxation of an external twist $\Delta \phi_{N-S} \sim 1$ radian. In this second scenario, the X-ray spectrum can be expected to soften following the burst, as the magnetosphere becomes more transparent to cyclotron scattering. These differences in post-flare behavior may serve to distinguish between the two possibilities.

We now discuss four pieces of observational evidence which bear upon this question. Although three seem to support the first hypothesis, the evidence is mixed. The most consistent interpretation is, perhaps, that the giant flares involve a redistribution of current in the magnetosphere, which decreases the magnetic shear locally while at the same time increasing the global twist.

1. The pulse profile of SGR $1900+14$ changed dramatically following the August 27 giant flare, simplifying to a single sinusoidal pulse from 4-5 sub-pulses (Woods et al. 2001a). This change has persisted even after the persistent X-ray flux returned to the baseline maintained before the flare (Woods et al. 2001a). This decoupling between the pulse profile and the total X-ray flux from SGR 1900+14, provides evidence that the energy source for the persistent emission is concentrated close to the star, inside the region where the pulse profile is established. Multiple scattering of $2-10 \mathrm{keV}$ photons at the electron cyclotron resonance will cause such a change in pulse profile, if the magnetic field is predominantly dipolar at a radius of $\sim 100 \mathrm{~km}$, and is a natural consequence of a twisting up of the external field during the flare. By contrast, in the second model, the simplified pulse profile would require the elimination of a non-axisymmetric component of the current during the flare. This is more difficult to arrange close to the star, where X-rays can be scattered at the ion cyclotron resonance.

2. Both giant flares (on 5 March 1979 and 27 August 1998) were initiated by a very intense $\sim 0.2-0.5 \mathrm{~s}$ pulse of hard X-rays and gamma rays (Mazets et al. 1979, 1999; Hurley et al. 1999a; Feroci et al. 1999), during which the bulk of the flare energy was probably deposited. This timescale is much longer than the light-crossing time of the central magnetosphere (where the energy in the twisted magnetic field is concentrated); but is similar to the time for a $\sim 10^{15} \mathrm{G}$ magnetic field to rearrange material in the deep crust and core of a neutron star across several kilometers. In the second model, a question of principle also arises as to how a very gradual build up of the external shear (over the estimated interval of $\sim 100$ years between giant flares in any one flare source) could lead to the sudden release of external magnetic energy on a timescale that is some 10 orders of magnitude shorter - without being initiated by a sudden yield or fracture in a rigid 
component of the star.

3. The persistent spectrum of SGR $1900+14$ softened measureably after the 27 August giant flare: the best-fit spectral index (pure power law) softened from $-1.89 \pm 0.06$ to $-2.20 \pm 0.05$ (Woods et al. 1999a). (Including a black body component, the best-fit index softened dramatically from $-1.1 \pm 0.2$ to $-1.8 \pm 0.2$.) This behavior is not consistent with a twisting up of the outer magnetosphere if the measured spectrum provides a fair measure of the angle-averaged spectrum and, in addition, if the non-thermal continuum is generated by resonant electron cyclotron scattering at $R \sim 50-100 \mathrm{~km}$. However, we have seen that regions of strong localized magnetic shear close to the star provide an alternative location for the source of this continuum - through e.g. resonant ion scattering (§屯, 5.4). The measured spectral change in SGR 1900+14 may therefore point to a more 'compact' scattering corona, within which the current relaxed even while it increased on more extended field lines following the flare.

4. Both giant flares were followed by multiple repeat bursts of a $\sim 1-7$ s duration (Golenetskii et al. 1987; Ibrahim et al. 2001) which is intermediate between the flares $(\sim 200-400 \mathrm{~s})$ and the much more common short SGR bursts ( 0.1 s: Gogus et al. 2001). These intermediate bursts released less than one percent of the energy of the giant flares, and most likely represent mild 'aftershocks' of the larger events. This behavior is possible to describe, in our axisymmetric magnetospheric model, if the release of energy is gated by the rigidity of the crust. The case of the 29 August 1998 burst from SGR 1900+14 - which had a similar peak luminosity to the pulsating tail of the August 27 flare but a duration 100 times shorter - is particularly instructive. Evidence for a trapped fireball comes from both bursts: from the shape of the declining light curve in the August 27 flare (Feroci et al. 2001); and from the presence of an extended faint, pulsating tail which followed the bright component of the August 29 burst (Ibrahim et al. 2001). This faint tail had a very hard spectrum which softened with time, and can be explained by the compression and heating of a small patch of the neutron star surface during the preceding burst. The high peak flux and short duration of the August 29 burst require that the magnetically confined fireball had a planar geometry, so that it cooled rapidly in one direction without decreasing significantly in area. The simplest interpretation here is that the August 29 burst involved a mild slippage of the crust along the same fault line which powered the preceding giant flare (Ibrahim et al. 2001). 


\subsubsection{Large-Amplitude Pulsations in the Giant Flares}

Within the 27 August 1998 flare itself, the X-ray flux showed pulsations of a very large amplitude, during all but the first 40 seconds (Hurley et al. 1999a; Feroci et al. 1999; Mazets et al. 1999). These pulsations repeated coherently at the 5.16-s spin period of the star, and the pulse profile maintained a complicated 4-peaked pattern during the intermediate portion of the burst (Feroci et al. 2001), which gradually simplified into a single pulse at the very end (Woods et al. 2001a). The 25-100 keV light curve recorded by Ulysses showed a large asymmetry between the first and second halves of the pulse profile, which was absent in the higher energy 40-700 BeppoSAX GRBM light curve (Feroci et al. 2001). The previous 5 March 1979 giant flare also showed large amplitude pulsations (Mazets et al. 1979). This narrow collimation of the X-ray flux, combined with the quasi-thermal flare spectrum, indicates a large angular variation in the optical depth.

The 'trapped fireball' model developed in Thompson \& Duncan (1995) has been successfully tested against the August 27 flare data in two respects (Feroci et al. 2001). After smoothing over the 5.16-s pulsations, the flare light curve can be well fit by the contracting surface of a magnetically confined $e^{ \pm}$fireball. In addition, the saturation of the best-fit X-ray temperature at $\sim 12 \mathrm{keV}$ during the last $\sim 350$-s of the burst is consistent with the freeze-out of photon splitting in a Comptonizing atmosphere, as predicted by Thompson \& Duncan (1995).

The cooling X-ray flux from the surface of a trapped fireball is concentrated close to the surface of the star, where the magnetic scattering opacity is greatly suppressed. It has been argued that, further from the fireball surface, the escaping X-ray flux will become collimated along partly open magnetic field lines, as the result of the strong inequality in the scattering opacity of the two X-ray polarization modes in a super-strong magnetic field (Thompson \& Duncan 1995, 2001). In particular, the opacity of the E-mode scales as $\sim B^{-2}$ and grows rapidly with radius. Thus, matter suspended higher in the magnetosphere by the hyper-Eddington flux can provide a dense scattering screen that is optically thick to Thomson scattering, and through which the X-rays can escape only by pushing the matter to the side. The burst light curve therefore provides information about the connectivity of the magnetic field lines close to the fireball surface. As the fireball shrinks, it connects with a smaller (and therefore more regular) portion of the magnetosphere - which could explain the reduced number of sub-pulses toward the end of the burst.

In the self-similar magnetospheric model which we have described, the resonant scattering opacity varies too smoothly with angle to explain the narrow collimation of the X-ray flux observed in both giant flares. Even during a giant flare, the photon flux is too weak to break open the closed field lines at a radius of $\sim 50-100 \mathrm{~km}$ 
(the position of the electron cyclotron resonance of a $\sim 40 \mathrm{keV}$ photon). The energy density in the magnetic field exceeds that in the freely streaming X-rays by a large factor $\sim 10^{6}\left(L_{X} / 10^{42} \mathrm{erg} \mathrm{s}^{-1}\right)^{-1}(\hbar \omega / 40 \mathrm{keV})^{4 / 3}\left(B_{\text {pole }} / 10^{14} \mathrm{G}\right)^{2 / 3}$ at the resonant surface, and so the radiation pressure imparts only a negligible distortion to the field lines. Thus, we must consider whether a sudden increase in the magnetospheric current during the first $\sim 1$ second of the flare (when most of the flare energy was probably released into the magnetosphere) could force the multiple X-ray beams to be re-scattered at the electron cyclotron resonance, and thereby isotropized.

During an outburst, the current-carrying electrons feel an enormous drag force where their cyclotron energy lies in the X-ray range, independent of the sign of the current (Appendix B). In the quiescent state, the electrons move in opposite directions (both toward the neutron star and away from it) on different portions of a closed current loop. However, charged particles may be injected into the magnetosphere at a much higher rate during an SGR outburst, than they need be to support the current associated with a static twist. The quiescent charge flow is given by eq. (18), and can be converted to a kinetic luminosity

$$
L_{\text {matter }} \sim \frac{I(R)}{e} m_{p} c^{2}=1 \times 10^{35}\left(\frac{B_{\text {pole }}}{10^{14} \mathrm{G}}\right)^{1 / 3}\left(\frac{\hbar \omega}{\mathrm{keV}}\right)^{2 / 3}\left(\frac{R_{\mathrm{NS}}}{10 \mathrm{~km}}\right) \quad \mathrm{erg} \mathrm{s}^{-1} .
$$

(Here we have expressed the resonant radius in terms of the cyclotron photon energy.) This luminosity is comparable to the persistent X-ray luminosity (see eq. 34]), but is less than one part in a million of the bursting luminosity. In a giant flare, the radiation pressure is high enough to advect matter outward at relativistic speed from the heated neutron star surface (Appendix B; Ibrahim et al. 2001). Only a minuscule fraction of the bursting luminosity need be carried by this entrained matter, in order to supply the charges which support a static current flowing along the twisted magnetic field.

The current can be maintained by a small differential drift of the ions with respect to the electrons and photons, in the presence of a dense wind from the neutron star surface. The cyclotron energy of the ions is too small to couple them tightly to the X-rays at the same radius where the electrons are tightly coupled. This allows the X-rays to flow outward, largely unimpeded, independent of the sign of the current. However, because the electrons are tightly coupled to the photons, a multipolar pattern can be maintained in the X-ray flux only if the dense matter transported to large radius reflects the multipolar pattern of the magnetic field close to the source.

It should also be noted that the pulse profile is measured in a much higher energy range during the flare (25-100 keV for Ulysses and 40-700 for BeppoSAX) than it is in quiescence 
(typically $2-10 \mathrm{keV}$ ). As a result, the pulse profile is more sensitive to the presence of higher magnetic multipoles during outburst, than in quiescence.

\subsubsection{Implications of the Variable Quiescent Pulse Profile of SGR 1900+14}

The 2-10 keV pulse profile of SGR $1900+14$ was complicated and multi-peaked before the 27 August 1998 giant flare (Hurley et al. 1999c). Our explanation for the change in the pulse profile following the flare (to a single sinusoidal pulse) involves a re-scattering of the X-rays at the electron-cyclotron resonance. If during the flare an additional twist was implanted in the magnetosphere, then there was at the same time an increase in the current density and the optical depth to resonant scattering.

The smooth, single pulse observed following the flare implies a simple - predominantly dipolar - geometry of the poloidal magnetic field at a distance of $\sim 50-100 \mathrm{~km}$ from the neutron star. We can then derive an upper bound to the strength of the surface quadrupole.

Neglecting departures from axisymmetry and reflection symmetry, the poloidal field can be decomposed as

$$
B_{\theta}(R, \theta=\pi / 2)=\sum_{\ell=1}^{\infty} B_{\ell}\left(R_{\mathrm{NS}}\right)\left(\frac{R}{R_{\mathrm{NS}}}\right)^{-(\ell+2)}
$$

at the magnetic equator. Here, $B_{1}=\frac{1}{2} B_{\text {pole }}$, and higher $\ell$ represent higher multipoles. We require that the quadrupole $\ell=2$ remain weaker than the dipole $\ell=1$ at the electron cyclotron resonance of a $10 \mathrm{keV}$ photon. Expressing the resonant radius as $R_{\mathrm{res}} / R_{\mathrm{NS}}=\left(B_{1} / B_{\mathrm{QED}}\right)^{1 / 3}(\hbar \omega / \mathrm{keV})^{-1 / 3}$, one deduces

$$
\frac{B_{2}\left(R_{\mathrm{NS}}\right)}{B_{1}\left(R_{\mathrm{NS}}\right)} \lesssim 5\left[\frac{B_{1}\left(R_{\mathrm{NS}}\right)}{10^{14} \mathrm{G}}\right]^{1 / 3}\left(\frac{\hbar \omega}{10 \mathrm{keV}}\right)^{-1 / 3} .
$$

The complicated 2-10 keV pulse profile observed before the August 27 flare then has two explanations in our model. The first possibility is that, even before the flare, the X-ray flux was reprocessed by resonant scattering off electrons at $R \sim 50-100 \mathrm{~km}$, but that the current was not axisymmetric. The second possibility is that the current flowing along extended field lines was small, so that the complicated pulse profile resulted from anisotropic emission and scattering close to the source. For example, the ion cyclotron resonance lies in the X-ray range near the surface of a magnetar, where the field is probably dominated by higher multipoles (\$5.3).

These two models lead to differing conclusions about the overall strength of the magnetic field at the surface of SGR $1900+14$. Polar electrical currents will accelerate the 
rate of spindown with respect to a simple magnetic dipole, and reduce the surface field inferred from the measured period and period derivative (see \$6). The rapid spindown of the SGRs 1900+14 and 1806-20 compared with the AXPs, and the displacement of SGR $1900+14$ from the center of the nearest supernova remnant, suggests that their spindown is transiently accelerated (Thompson et al. 2000). Such a temporary increase in the rate of spindown could be effected through a global twist imparted to the magnetosphere.

In the absence of a global twist, SGR $1900+14$ is inferred to have a polar dipole magnetic field $B_{\mathrm{MDR}} \sim 2 \times 10^{15} \mathrm{G}$, based on the rate of spindown before the giant flare (Kouveliotou et al. 1999). The presence of higher magnetic multipoles (needed to explain the complicated angular pattern of resonant ion scattering) then guarantees yet stronger surface fields, and allows multiple ion scattering to occur up to a high energy cutoff of $\sim 40\left(B_{\text {surface }} / 3 B_{\mathrm{MDR}}\right) \mathrm{keV}$.

A check of these ideas is provided by a relatively short $3.5 \mathrm{~s}$ burst emitted by SGR 1900+14 on 29 August 1998, two days after the giant flare. This burst was followed by a faint $\left(L_{X}<10^{37} \mathrm{erg} \mathrm{s}^{-1}\right)$ and very extended tail, which lasted more than $1000 \mathrm{~s}$ and gradually merged with the persistent emission (Ibrahim et al. 2001). The $2-20 \mathrm{keV}$ pulse profile in this tail had a similar shape, and maintained a constant relative phase, with the later persistent emission (Palmer 2001). Even at its peak, the radiative flux of the tail did not exceed that of the persistent emission 14 by more than an order of magnitude. Thus, the pulse profile should be controlled by the same resonant scattering processes in the decaying tail, as it is in the later persistent emission, and the observed constant phase alignment of the pulse is expected.

\section{Implications for SGR/AXP spindown}

The measured spindown of SGR 1806-20 (Kouveliotou et al. 1998) and SGR 1900+14 (Kouveliotou et al. 1999; Woods et al. 1999c; Marsden et al. 1999) corresponds to a polar dipole field $B_{\text {pole }} \simeq 2 \times 10^{15} \mathrm{G}$. In the presence of a net twist, the external magnetic field drops off more slowly than $\sim R^{-3}$ (Fig. 2). The field strength $B\left(R_{\mathrm{lc}}\right)$ at the speed of light cylinder $R_{\mathrm{lc}}=c P / 2 \pi$ is then stronger than a pure dipole, $B_{\theta}\left(R_{\mathrm{lc}}\right) / B_{\text {pole }}=\frac{1}{2} p F(0)\left(R_{\mathrm{lc}} / R_{\mathrm{NS}}\right)^{-(2+p)}$. Since the rate of loss of rotational energy is $I \Omega \dot{\Omega} \sim B_{\theta}\left(R_{\mathrm{lc}}\right)^{2} R_{\mathrm{lc}}^{2} c$, the flaring of the field causes the spindown rate to increase. Equivalently, the real polar surface field is weaker

\footnotetext{
${ }^{14}$ The August 29 burst itself occurred during a period of enhanced persistent emission following the August 27 giant flare (Woods et al. 2001a); this comparison is made with the amplitude of the persistent emission recorded just before the August 29 burst.
} 
than the field inferred from the measured $\dot{P}$ and $P$ using the standard magnetic dipole formula. Compared directly with the magnetic dipole value, one finds

$$
\frac{B_{\text {pole }}}{B_{\text {pole }}(p=1)}=\frac{1}{p F(0)}\left(\frac{c P}{2 \pi R_{\mathrm{NS}}}\right)^{p-1} .
$$

Notice that there is no direct dependence on $\dot{P}$ in this expression. An additional consequence is to reduce the braking index below the dipole value,

$$
n=\frac{\ddot{\Omega} \Omega}{(\dot{\Omega})^{2}}=2 p+1 .
$$

The ratio (45) is plotted in Fig. 6 for various spin periods. The true polar field is smaller by a factor $\simeq \frac{1}{3}$ when the outer magnetosphere has a twist $\Delta \phi_{\mathrm{N}-\mathrm{S}}=1$ radian, for spin periods in the range $3-10 \mathrm{~s}$; whereas a twist $\Delta \phi_{\mathrm{N}-\mathrm{S}}=1.5$ radian leads to a reduction of one order of magnitude in $B_{\text {pole }}$. This model has the further implication that, for a fixed polar field, the spindown rate increases with the optical depth to resonant scattering, and hence with the hardness of the persistent X-ray spectrum. Indeed, the active SGRs 1806-20 and 1900+14 both have higher $\dot{P}$ and harder X-ray spectra than any AXP - a trend which has been further quantified by Marsden and White (2001) for the combined population of SGR and AXP sources. The quiescent SGR 0526-66 has a softer spectrum (Kulkarni et al. 2001). We predict that its spindown rate, when measured, will be intermediate between these sources and the AXPs.

It should be emphasized that if the spindown of a magnetar is persistently accelerated in this manner (so that the magnetospheric twist remains constant), then its characteristic age hardly differs from the magnetic dipole value. The spin frequency decreases as $\Omega(t) \propto t^{-2 p}$, and so the characteristic age is larger than $P / 2 \dot{P}$ by a factor $1 / p$. In the case of the AXP 1E 1841-045, the near equality between the $4 \times 10^{3}$ yr characteristic age and the age of the surrounding Kes 73 (Gotthelf et al. 1999) does not imply that its magnetic field must be close to a true dipole.

It has been previously noted (Thompson \& Blaes 1998; Harding, Contopoulos, \& Kazanas 1999; Thompson et al. 2000) that persistent seismic activity in a magnetar can also increase the rate of spindown with respect to a vacuum magnetic dipole - by triggering a fluctuating current in the magnetosphere which drives an outward flux of particles and Alfvén waves. The real polar field of SGRs 1806-20 and 1900+14 is reduced by a factor $\sim 3$ with respect to the one inferred from to the magnetic dipole formula, if the persistent seismic luminosity is equal to the observed X-ray luminosity of $\sim 10^{35} \mathrm{erg} \mathrm{s}^{-1}$. The plerionic synchrotron emission powered by a persistent particle wind of luminosity $\sim 10^{35} \mathrm{erg} \mathrm{s}^{-1}$ 
could, in fact, have escaped detection. Measurements of spindown in SGR $1900+14$ or SGR 1806-20 do not, however, show a direct correlation between the rate of spindown and bursting activity - with the noticeable exception of the August 27 giant flare itself (Woods et al. 1999c, 2001b). For this reason, static magnetospheric currents seem a more promising source of non-uniform spindown in the SGR and AXP sources.

Ejection of a large number of particles during a giant flare would cause a transient spindown of a soft gamma repeater. As we now show, the cumulative torque is larger if the external field is twisted, than if it is dipolar close to the star. The spin period of SGR $1900+14$ did indeed increase by $\Delta P / P=1 \times 10^{-4}$ (in comparison with the extrapolation of the previously measured spindown) within three months of the 27 August 1998 giant flare (Woods et al. 1999a). However, the torque calculated assuming a dipolar (near) field is too small by an order of magnitude if $B_{\text {pole }} \sim 10 B_{Q E D}=4.4 \times 10^{14} \mathrm{G}$, and if the particle energy $\Delta E$ and duration $\Delta t$ of the outflow are normalized to the energy and duration of the X-ray outburst (Thompson et al. 2000). Estimating

$$
I \frac{\Delta \Omega_{\mathrm{NS}}}{\Omega_{\mathrm{NS}}} \simeq-\frac{2}{3 c^{2}} \Delta E R_{A}^{2}
$$

where the Alfvén radius is determined by balancing the ram pressure of the particles with the magnetic tension,

$$
\frac{\Delta E / \Delta t}{4 \pi R_{A}^{2} c}=\frac{B^{2}\left(R_{A}\right)}{4 \pi}=\frac{B_{\mathrm{pole}}^{2}}{4 \pi}\left(\frac{R_{A}}{R_{\mathrm{NS}}}\right)^{-2(2+p)},
$$

one finds that the net torque is increased by a factor

$$
\frac{(\Delta P / P)_{p=0.8}}{(\Delta P / P)_{p=1}}=4\left(\frac{B_{\mathrm{NS}}}{10 B_{\mathrm{QED}}}\right)^{1 / 9}\left(\frac{\Delta E}{10^{44} \mathrm{erg}}\right)^{-1 / 18}\left(\frac{\Delta t}{400 \mathrm{~s}}\right)^{1 / 18}
$$

when $p$ is reduced from 1 to 0.8 . This brings the calculated torque close to the observed value if $B_{\text {pole }} \sim 10 B_{\mathrm{QED}}$.

\subsection{Narrow Distribution of SGR/AXP Spin Rates}

The SGR sources have spin periods measured四 in the range 5-8 s. The distribution of spin periods for the AXP sources is remarkably similar: 6-12 s (e.g. Mereghetti 2000).

\footnotetext{
${ }^{15}$ Derived from the persistent emission for SGR 1806-20 (Kouveliotou et al. 1998), from the persistent emission and 27 August 1998 giant flare for SGR 1900+14 (Hurley et al. 1999a,b; Feroci et al. 1999; Mazets et al. 1999), and from the 5 March 1979 giant flare for SGR 0526-66 (Mazets et al. 1979).
} 
While the number of sources is small enough that the detection of much shorter spin periods is not surprising, even if the sources are born spinning much more rapidly, it is surprisingly narrow given the wide range of characteristic ages $P / \dot{P}-$ from $1-3 \times 10^{3}$ yr for SGRs $1806-20$ and $1900+14$ up to $4 \times 10^{5}$ yr for the AXP 1 E $2259+586$. It has been suggested, as a result, that the spindown of the active SGRs is transiently accelerated, and that the spindown of $1 \mathrm{E} 2259+586$ (which sits near the center of the much younger SNR CTB 109) has decayed significantly from its long term average (Thompson et al. 2000). Clearly transient acceleration is possible in this model if the AXPs are (mostly) dormant SGRs, and the external magnetic field is twisted up during periods of burst activity.

The narrow range of spin periods is suggestive of some physical process which limits the spindown rate beyond a period of $\sim 8$ seconds. We would like to point out that this period is remarkably close to the upper envelope of the distribution of spin periods in the known radio pulsar population.

The magnetospheric model which we have outlined provides a motivation for a reduction in torque above a critical spin period, where the potential drop through the magnetosphere is no longer high enough to trigger a pair cascade through emission of curvature $\gamma$-rays. In the absence of pair creation, the space-charge limited flow along open magnetic field lines will generate, in one hemisphere, a toroidal magnetic close to the magnetic axis with the opposite sign to the more global toroidal field (Fig. [). Fast reconnection of these opposing toroidal fields would have the effect of forcing a relaxation of the static twist inside the speed of light cylinder. In other words: beyond the pair death line, the intermediate regions of the corotating magnetosphere probably cannot maintain a static twist. Current will diffuse away from the region closest to the star (where the most of the current is concentrated in our self-similar solutions: eq. [18]) only on the relatively long timescale (38).

We should emphasize that we have not yet been able to demonstrate unambiguously the opposite effect: that pair creation will act to stabilize a large-scale twist. Pair creation does cause a huge multiplication in the number of charges flowing outward on open field lines, so that the pair-loaded plasma is capable of maintaining a net Goldreich-Julian charge density - even while the axial current flows in the opposite direction to the original space-charge limited flow (\$3.1). Whether the global current flow actually takes advantage of this effect is a question that we cannot presently address from first principles. 


\section{Summary}

We have shown that several properties of magnetar candidates in their quiescent states become easier to understand if the neutron star's magnetic field is globally twisted. These properties are directly affected in a correlated manner by persistent magnetospheric currents. The observed X-ray pulse profile and spectrum are modified by resonant cyclotron scattering and the spindown torque is increased (for a fixed surface field strength) over the standard vacuum dipole formula.

We have idealized the magnetosphere as a twisted dipole, and constructed self-similar solutions to the force-free equation. The self-similar ansatz requires that the surface flux density and the current density (related to the twist of the field lines) have a particular dependence on the magnetic latitude which changes shape depending on the strength of the current. As the net twist between the north and south magnetic hemispheres $\Delta \phi_{\mathrm{N}-\mathrm{S}}$ increases from 0 to $\pi$, the external field continuously interpolates between a dipole and a twisted, split monopole. Since axisymmetric shear deformations of an axisymmetric star do not change the angular distribution of $B_{R}$, actual deformations of a magnetar must be represented by some non-linear combination of these solutions. This also suggests that the magnetosphere of a neutron star may, in practice, not be able to maintain a twist larger than $\Delta \phi_{\mathrm{N}-\mathrm{S}} \sim 1-1.5$ radians (above which the distribution of flux with polar angle begins to differ significantly from a pure dipole).

Our principal conclusions can be summarized as follows:

1. It has previously seemed difficult to make deductions about the configuration of the magnetic field in the SGR and AXP sources: one obtains evidence for the presence of higher multipoles from the light curves of the giant flares (Feroci et al. 2001; Thompson \& Duncan 2001) but not much more than that. The existence of magnetars was motivated by considerations of magnetic field amplification through dynamo activity in young, convective neutron stars: a large-scale helical dynamo is possible when the initial spin period is shorter than $\sim 3$ msec (the convective overturn time of nuclear matter from which neutrinos are escaping with a luminosity $L_{\nu} \gtrsim 10^{52} \mathrm{erg} \mathrm{s}^{-1}$ ) (Duncan \& Thompson 1992; Thompson \& Duncan 1993). Nonetheless, the connection between this theoretical dynamo model and the (now) slowly rotating SGR and AXP sources has seemed tenuous. The new results presented in this paper - which indicate the presence of strong internal toroidal fields in the SGR and AXPs - show that this theoretical picture has, at least, a degree of self-consistency.

2. A persistent current can be maintained by electrons and ions stripped from the neutron star surface, if the elemental composition is light (e.g. hydrogen, helium, or carbon). The impact of the returning ions at the cathode region of the neutron star surface, 
and the downward acceleration of the returning electrons at the anode surface (where the ions are accelerated upward) generates a luminosity $L_{X} \sim 10^{35}\left(B_{\text {pole }} / 10^{14} \mathrm{G}\right) \mathrm{erg} \mathrm{s}^{-1}$ in surface X-ray emission if the entire magnetosphere is twisted. This luminosity provides a lower bound to the electromagnetic output of a neutron star with an actively decaying magneic field and is, by coincidence, comparable to the passive X-ray flux powered by ambipolar diffusion in the neutron star core (TD96; Heyl \& Kulkarni 1998). The rate of surface heating would be lower by a factor $\sim \gamma_{e} m_{e} / m_{p}$ if the current were carried by relativistic $e^{ \pm}$pairs of Lorentz factor $\gamma_{e}$.

A significant rate of surface heating leads to the interesting possibility of an emission line at the surface ion cyclotron frequency. The magnetospheric ions are stopped mainly by ion collisions in magnetic field much stronger than $B_{\mathrm{QED}}$. The stopping depth is higher than in the case of a non-magnetic atmosphere, and the broad-band spectrum will, as a result, be closer to a black body than in the weak-field regime analyzed by Zel'dovich \& Shakura (1969) and Deufel, Dullemond, \& Spruit (2001).

3. A significant optical depth to resonant cyclotron scattering, $\tau_{\text {res }} \sim$ $(v / c)^{-1}\left(B_{\phi} / B_{\theta}\right)_{\theta=\pi / 2}$, is generated by the current carriers at the magnetic equator. This optical depth is anisotropic and approaches zero at the magnetic axis, where the equilibrium current density is $J(\theta) \propto \theta^{2}$. When the twist is large, $\left(B_{\phi} / B_{\theta}\right)_{\theta=\pi / 2}=O(1)$, photons will experience multiple resonant scattering, forming a high energy non-thermal tail. At a fixed $L_{X}$, the hardness of this tail will increase with the strength of the overall twist imparted to the magnetosphere, and so one obtains an explanation for the trend of increasing spectral hardness with overall burst activity in the combined population of SGR and AXP sources.

In our self-similar model, $\tau_{\text {res }}$ is independent of the charge and mass of the particles, as well as the resonant radius. The large difference in the charge/mass ratio of electrons and ions therefore allows a magnetar to have more than one scattering corona, localized at quite different radii: $R \sim 50-100 \mathrm{~km}$ for electrons and $\sim 10-20 \mathrm{~km}$ for ions. Ions will resonantly scatter X-rays only below the (Doppler-shifted) surface cyclotron frequency, and so the spectral tail generated by ion cyclotron scattering will be cut off at a much lower frequency than in the case of resonant electron scattering. Measurements of the persistent emission above $\sim 30 \mathrm{keV}$ can test the relative importance of the two mechanisms.

The overall similarity in the luminosities of the thermal and non-thermal components of SGR and AXP spectra has a simple interpretation in this model, but would be more difficult to understand if the non-thermal emission arose from an independent radiative process (such as synchrotron or curvature emission) in the magnetosphere. For these sources, the mechanism of multiple cyclotron scattering also has significant advantages over 
non-resonant Comptonization in a thin surface layer which is heated by magnetospheric charges (Zel'dovich \& Shakura 1969; Deufel et al. 2001). Aside from the relatively soft spectrum of the surface emission expected in a strong magnetic field, it will also be noted that the intrinsically brightest soft gamma repeater 0526-66 has a softer spectrum than the other three SGRs (each of which are $10-30$ times less luminous; Kulkarni et al. 2001).

4. The observed pulse profile is strongly modified by resonant cyclotron scattering. Three effects enter here: the strong anisotropy in the optical depth to electron cyclotron scattering, the aspherical shape of the resonant surface, and the doppler beaming of the scattered radiation resulting from the bulk motion of the charge carriers. In addition, the flux of thermal seed photons generated by the surface impact of magnetospheric charges is strongly anisotropic even in this self-similar model. Not only is the surface current inhomogeneous, but a larger fraction of the radiative flux will be carried by the O-mode (which is beamed along the local magnetic field) than is the case in passively cooling neutron stars. This second effect is enhanced if the magnetospheric current is concentrated on extended field lines (e.g. TD96).

5. Soft Gamma Repeater flares provide prima facie evidence for sudden variations in the magnetic field, and therefore in the electrical currents flowing outside the star (Thompson et al. 2000). We have shown that the energy available in the external field can maintain these currents for as long as $\sim 30\left(B_{\text {pole }} / 10^{14} \mathrm{G}\right) \mathrm{yr}$, if the current is supported by electrons and ions stripped from the neutron star surface. It is interesting to note, in this regard, that SGR 0526-66, which has been quiescent as a burst source since 1983, has a persistent X-ray spectrum which is strongly non-thermal but at the same time significantly softer than the actively bursting SGRs (Kulkarni et al. 2001). This model also provides an explanation for the simplified pulse profile observed in the persistent emission of SGR 1900+14 following the 27 August 1998 giant flare, which was maintained even after the $\mathrm{X}$-ray flux returned to the baseline value observed before the flare. We obtain a valuable constraint on how the magnetic field was modified during the flare: the current flowing along extended magnetic field lines actually increased during the flare, suggesting that it was triggered by the release of sub-surface stresses.

6. Given a fixed polar magnetic field $B_{\text {pole }}$, the observed rate of spindown will grow as the external field is twisted up, and there is an increase in the fraction of the field lines which open out across the speed-of-light cylinder. Equivalently, the real surface polar field is reduced by a factor 3 for a net twist $\Delta \phi_{\mathrm{N}-\mathrm{S}}=1$ radian, in comparison with the dipole formula (Fig. 6).

7. Consideration of the stability of a twisted, force-free magnetosphere leads to the requirement that, close to the magnetic axis, the current flows in the same direction on 
both closed and open field lines. Such an alignment of the currents can be achieved only if the charge flow is pair-loaded on open field lines - which allows a differential drift between positive and negative charges to maintain a current opposite to $\rho_{G J} c \hat{R}$ in one hemisphere. There is, in turn, a limiting spin period beyond which the rate of spindown can no longer be accelerated with respect to an orthogonal vacuum dipole. If the pair cascade cuts off at a spin period comparable to the maximum observed in the known radio pulsar population, then one obtains an explanation for the observed narrow distribution $(P=6-12 \mathrm{~s})$ of AXP spins, and the similarly narrow distribution $(P=5-8 \mathrm{~s})$ of SGR spins. Direct evidence for such a decay in the torque is provided by the anomalous pulsar $1 \mathrm{E} 2259+586$, which has a characteristic age at least a factor of 10 larger than the age of the SNR in which it resides (Thompson et al. 2000).

\subsection{Relation between the SGRs, AXPs, and the Soft X-ray Pulsars}

In this section, we comment on the connection between the Soft Gamma Repeaters, Anomalous X-ray Pulsars, and their possible cousins, a growing group of Soft X-ray Pulsars.

\subsubsection{Polar Dipole Fields of the SGRs/AXPs and their Relation to Radio Pulsar Fields}

The polar magnetic fields 1900+14 (Kouveliotou et al. 1998, 1999, Woods et al. 2001b) are inferred to be $B_{\text {pole }}=1-3 \times 10^{15} \mathrm{G}$ from the standard magnetic dipole formula. These fields lie a factor of 10-30 above the strongest fields measured in the radio pulsar population, $B_{\text {pole }}=10^{14} \mathrm{G}$ (Camilo et al. 2000). The polar fields inferred analogously for the AXPs are continuous with the pulsar distribution: the source $1 \mathrm{E} 2259+586$ has a long characteristic age $P / 2 \dot{P}=2.3 \times 10^{5}$ yr and polar dipole field $1.2 \times 10^{14} \mathrm{G}$. However, most of the AXPs have nominal dipole fields 5-10 times this value.

In our model, the actual polar magnetic fields of these sources will lie below the classical magnetic dipole value. A reduction of $\sim 3-10$ is plausible in some sources (corresponding to net twist angles of $\sim 1-1.5$ radians). The higher spindown rates measured in the SGRs, as compared with the AXPs, could simply represent a greater degree of magnetospheric twist imparted by deformations of the magnetic field that are associated with bursting activity. Indeed, the two AXPs with the fastest spindown also have the hardest persistent

\footnotetext{
${ }^{16}$ This polar field exceeds by a factor 2 the average surface field usually quoted in the radio pulsar literature.
} 
X-ray spectra (e.g. Table 2 of Kaspi et al. 2001). This positive correlation between spindown rate and spectra becomes even stronger when the SGRs and AXPs are lumped together (Marsden \& White 2001). Nonetheless, some distribution of surface fields is almost certainly present in the AXP and SGR populations, and it is plausible that some of the quiescent sources really do have weaker surface fields than the bursting sources.

Additional physical constraints on the dipole fields of the SGR sources come from these independent lines of argument:

i) Confinement of the relativistically hot plasma which powered the pulsating tails of the two giant flares requires magnetic fields stronger than $10^{14}\left(E / 10^{44} \mathrm{erg}\right)^{1 / 2} \mathrm{G}$ (Thompson \& Duncan 1995). This argument has been generalized to allow for the possibility that the dipole is offset from the center of the star, which would reduce the magnetic moment corresponding to a fixed plasma energy $E$ (Thompson \& Duncan 2001). Even including this effect, one deduces $B_{\text {pole }} \gtrsim 10^{14} \mathrm{G}$, because a compact fireball would have a very high internal temperature $T \gg 1 \mathrm{MeV}$ and would lose its energy rapidly to neutrino radiation through $e^{+}+e^{-} \rightarrow \nu+\bar{\nu}$ (instead of the observed X-ray flux).

ii) Transient spindown of SGR $1900+14, \Delta P / P=1 \times 10^{-4}$, was observed within 3 months of the 27 August 1998 giant flare (Woods et al. 1999c). A plausible mechanism for this torque involves a particle wind during the giant flare itself, combined with scattering of the X-rays by matter suspended in the magnetosphere near $R \sim 200 \mathrm{~km}$ (where the momentum flux is high enough to break open the magnetic field lines). If the magnetic field were dipolar inside this 'Alfvén' radius, then the maximum torque would be $\Delta P / P \simeq 10^{-5}\left(B_{\text {pole }} / 10 B_{Q E D}\right)$ (Thompson et al. 2000). However, if the magnetic field were twisted close to the star, then the Alfvén radius would increase. The net torque would be brought close to the observed value if $B_{\text {pole }} \gtrsim 5 \times 10^{14} \mathrm{G}$ (eq. [49]).

iii) The surface X-ray flux predicted by our self-similar model (eq. [34]) is comparable to the observed luminosities of the SGR and AXP sources if $B_{\text {pole }} \sim 10^{14} \mathrm{G}$, but is excessively large if $B_{\text {pole }}$ is as large as $10^{15} \mathrm{G}$. However, it should be emphasized that the internal stresses acting on the crust of a magnetar may be more localized, and its entire magnetosphere need not be twisted. The polar field could be as strong as $\sim 10^{15} \mathrm{G}$ if the current were intermittent and flowed only over a fraction of the neutron star surface.

All of these arguments point to dipole fields stronger than $10^{14} \mathrm{G}$. A polar field several times the strongest pulsar field appears needed to explain a transient spindown $\Delta P / P=1 \times 10^{-4}$ of SGR $1900+14$ by an outflowing wind during the 27 August flare. The quiescent SGR 0526-66 has a softer spectrum (Kulkarni et al. 2001). We predict that its spindown rate, when measured, will be intermediate between these sources and the AXPs. 
We re-emphasize that the actual surface fields of the SGR sources are probably much stronger than the (corrected) dipole fields: the complicated pulse profile observed during the 27 August flare provides direct evidence for the presence of higher multipoles (Feroci et al. 2001; Thompson \& Duncan 2001). In addition, only magnetic fields stronger than $\sim 4 \times 10^{15} \mathrm{G}$ will experience rapid ambipolar diffusion through the core of a magnetar (TD96; Heyl \& Kulkarni 1998).

To summarize: our model indicates that the distribution of true polar (dipole) magnetic fields of the SGRs/AXPs is significantly narrower than the classical dipole formula would suggest. The range of spindown rates is broadened by magnetospheric currents. The true polar fields of the SGRs and AXPs are continuous with the distribution of radio pulsar fields; but are probably stronger than $10^{14} \mathrm{G}$ in some sources. Radio pulsars correspond to those sources in which the toroidal field is either absent, or too weak to shear the crust. Furthermore if an accelerated torque in the SGR/AXP sources is associated with an active pair cascade on open field lines, then strong-B fields do not, in themselves, suppress pair creation: there must be a greater similarity in this regard between active magnetars and ordinary radio pulsars, than some calculations have suggested.

\subsubsection{Relation between the SGRs and AXPs}

The SGRs are distinguished from the AXPs by the emission of bright X-ray outbursts and, in their quiescent states, harder X-ray spectra and faster spindown. We have shown that the last two properties can be explained by a greater degree of twist in the external magnetic field. In addition, the sudden untwisting of an (internal) magnetic field provides an attractive mechanism for powering the giant flares of the SGRs. This model suggests that some AXPs may be dormant SGRs: indeed, the SGRs go through long periods of quiescence (the LMC source SGR 0526-66 has not been observed to burst since 1983; Golenetskii et al. 1987).

Even in such a unified description of the SGRs and AXPs, the question remains as to whether one type of activity typically precedes the other in a given source, or whether instead an AXP will undergo sporadic intervals of SGR activity which are separated by periods of silence as a burst source. In fact, some AXPs may never manifest SGR behavior. It has been suggested by Gaensler et al. (2001) that the AXPs are characteristically younger than the SGRs, because 3 of the 6 AXP sources are situated very close to the centers of supernova remnants. On the other hand, the other 3 AXPs do not have obvious SNR counterparts, and one soft gamma repeater (SGR 1806-20) sits close to the center of the radio nebula G10.0-0.3 (Kulkarni et al. 1994). Circumstantial evidence for high 
proper motions in two SGRs comes from the projected position of SGR 0526-66 near the edge of the LMC remnant N49 (Cline 1982), and the position of SGR 1900+14 just outside SNR G42.8+0.6 (Hurley et al. 1999b). A systematic difference in proper motions between the AXPs and SGRs, if real, is an important clue to the conditions which give rise to these sources, but our model does not offer any unambiguous suggestion for what that difference may be.

The short spindown ages of SGRs $1900+14$ and $1806-20(P / \dot{P}<3000$ yrs: Kouveliotou et al. 1998, 1999; Woods et al. 2001c) seem to provide evidence, at first sight, that these sources are younger than most of the AXPs. This impression could, however, be an artifact of a sufficiently strong twisting of the external field. If the sources are in fact older (as their positions with respect to the nearest SNR would suggest) then their spindown must be persistently but transiently accelerated with respect to the AXP population (Thompson et al. 2000). Combining the two populations, any AXP must spend at most $\sim 25 \%$ of its $\sim 10^{4}-10^{5}$ yr lifetime as a bright X-ray source in an SGR mode (Thompson et al. 2000). However, the length of any given interval of SGR activity is poorly constrained at present: it must be at least $10-20$ yrs, but could easily be much longer than that.

We conclude that no unambiguous sequence of SGR and AXP activity is discernable from the data, in part because the spindown torques of the bursting sources appear to be accelerated transiently, and also because a wide range of proper motions may exist in the combined population of SGRs and AXPs. A plausible scenario is one in which a portion of the AXP population undergoes intermittent periods of bursting activity. It should be kept in mind that SGR activity may be concentrated during a particular range of ages, when the crust of the star is colder and more brittle. Because the spindown torque may be increased substantially by global magnetospheric currents, the true polar fields of the actively bursting SGRs need not lie at the extreme high end of the magnetar population.

This model is testable by long-term monitoring of the spin of an SGR source, after it ends a period of bursting activity. Thus, continuous (phase-connected) monitoring of these sources is crucial to the unraveling of the relation between the SGR and AXP phenomena.

\subsection{Connection between the SGRs and AXPs and nearby Soft X-ray Pulsars}

The nearby soft X-ray pulsars RX J0420.0-5022, RX J0720.4-3125, and RBS 1223 have spin periods $(P=22.7,8.37$ and 5.2 s; Neuhäuser \& Trümper 1999) remarkably close to the SGRs and AXPs. It has been noted (Heyl \& Hernquist 1998; Kulkarni \& van Kerkwijk 1998) that these sources may be aged magnetars. They are much fainter X-ray 
sources than the SGRs and AXPs and could, at earlier times, have been observable either as radio pulsars or as SGRs/AXPs. (The microphysical heating mechanism most plausibly is a combination of ohmic decay and Hall deformations in the neutron star crust, because ambipolar diffusion of a magnetic field through the core should be largely frozen when the surface temperature is as low as $\sim 60-100 \mathrm{eV}$.) If these objects are evolved from the SGR/AXP population, then they provide further evidence for a decay of the torque beyond a characteristic spin period. The recent possible detection of rapid spindown in the source RBS 1223 implies a surprisingly short characteristic age of $\sim 10^{4}$ yrs (Hambaryan et al. 2001), which is not consistent with this simple scenario.

\subsection{Transient Effects}

This model also provides a basis for interpreting time-dependent effects observed in the SGR and AXP sources, including variations in flux and pulse profile (Iwasawa, Koyama, \& Halpern 1992; Woods et al. 2001a), and variations in torque (Paul et al. 2000; Kaspi et al. 2001; Woods et al. 2001b). Indeed, these transient effects probably provide the strongest constraints on the physical processes operating in the magnetospheres of the SGR and AXPs.

We are optimistic that progress in understanding the electrodynamics of neutron stars with actively decaying magnetic fields - magnetars - will occur more rapidly than has been the case with radio pulsars - for the simple reason that the observations place many more direct constraints on theoretical models.

We thank Phil Arras, Vicky Kaspi, Chryssa Kouveliotou, and Peter Woods for comments and stimulating discussions. CT acknowledges the support of the NSERC of Canada, and the Alfred P. Sloan Foundation. ML acknowledges the support of a CITA National Fellowship. CT and ML also thank the Institute for Theoretical Physics at the University of California at Santa Barbara (NSF grant PHY99-0749) for its support during the workshop on 'Spin, Magnetism and Rotation in Young Neutron Stars', when part of this work was done.

\section{A. Polarization Mode Exchange.}

In this appendix we show that even near the center of the cyclotron resonance, the dielectric properties of the magnetosphere are dominated by vacuum polarization. The 
plasma contribution to the refractive index is

$$
|n-1|_{\text {plasma }}=\frac{2 \pi Z e n_{Z} c}{B \Delta \omega}
$$

at a frequency $\omega=Z e B / M c \pm \Delta \omega$. (Here $Z e, M$ and $n_{Z}$ are the charge, mass and density of the resonant particles, which could either be electrons or ions.) Estimating $Z e n_{Z} \sim B / 4 \pi R$ through Ampère's equation, this gives

$$
|n-1|_{\text {plasma }} \sim \frac{1}{4 \pi}\left(\frac{\lambda}{R}\right)\left(\frac{\Delta \omega}{\omega}\right)^{-1},
$$

independent of $Z$ and $M$, but depending on the wavelength $\lambda=2 \pi c / \omega$ of the X-ray photon. By contrast, the vacuum contribution to the index of refraction is

$$
|n-1|_{\text {vacuum }}=K \alpha_{\mathrm{em}}\left(\frac{B}{B_{\mathrm{QED}}}\right)^{2} \sin ^{2} \theta=K \alpha_{\mathrm{em}}\left(\frac{\hbar \omega}{m_{e} c^{2}}\right)^{2} \sin ^{2} \theta,
$$

which we have evaluated at the electron cyclotron resonance $\hbar \omega=\left(B / B_{\mathrm{QED}}\right) m_{e} c^{2}$. In this expression $\alpha_{\mathrm{em}}=\frac{1}{137}$, and the constant $K=\frac{7}{90}$ for the O-mode and $\frac{2}{45}$ for the E-mode. The characteristic width of the resonance is determined by the thermal motion, $\Delta \omega / \omega \sim\left(k T / m_{e} c^{2}\right)^{1 / 2}=0.04(k T / \mathrm{keV})^{1 / 2}$. We conclude that the vacuum contribution is the larger by a factor $\sim 10^{6}(\hbar \omega / \mathrm{keV})^{3}(\Delta \omega / \omega)$ at the electron cyclotron resonance (radius $R \sim 100 \mathrm{~km}$ for magnetar-strength fields).

The good photon polarization states are linear in this regime, and both are absorbed and emitted at the cyclotron resonance (which interacts with an elliptically polarized mode). The net result is that cyclotron scattering, at both ion and electron resonances, will reduce the polarization of X-rays escaping the star in a direction almost parallel to the local magnetic field, and will induce a net linear polarization of X-rays escaping across closed magnetic field lines (with the polarization vector lying perpendicular to $\mathbf{B}$ ). Thus, measurements of the emergent X-ray polarization (Heyl \& Shaviv 2000) will provide a direct probe of the current flowing through the atmosphere of a magnetar.

\section{B. Resonant Cyclotron Force Vs. Gravity}

The radiation field is anisotropic everywhere in the magnetosphere, and a particle at rest will feel a force from resonant cyclotron scattering. We now show that if the surface $\mathrm{X}$-ray flux is powered self-consistently by the impact of magnetospheric charges (\$5.1), then the radiative force acting on electrons is typically large compared with gravity. The

conclusion is slightly more complicated for ions: in the quiescent state of an SGR or AXP 
source, the radiative force acting on them is typically small compared with gravity at the surface of the star. The radiative force remains weaker than gravity at greater distances if the spectral intensity $L_{\omega}$ increases with frequency below $\hbar \omega \sim 1 \mathrm{keV}\left(L_{\omega} \sim \omega^{\alpha}\right.$ with $\left.\alpha>0\right)$.

\section{B.1. Radiative Force}

The radiative force is easily estimated when the magnetic field is purely radial, and the radiation field is axially symmetric about $\mathbf{B}$. Because the re-emitted photon carries vanishing average momentum, this force is

$$
F_{\mathrm{rad}}=\int d \omega \int_{0}^{1} 2 \pi d(\cos \theta)\left[\frac{d L_{\omega}}{d \Omega} \cos \theta\right] \frac{\sigma_{\mathrm{res}}(\omega, \cos \theta)}{4 \pi R^{2} c}
$$

(we neglect the effect of the recoil; cf. Sincell \& Krolik 1992). Substituting $\sigma_{\text {res }}(\omega, \cos \theta)=\left(Z^{2} \pi^{2} e^{2} / M c\right)\left(1+\cos ^{2} \theta\right) \delta\left(\omega-\omega_{c}\right)$ near the cyclotron frequency

$\omega_{c}=Z e B / M c$, one finds (see Mitrofanov \& Pavlov 1982 for the case of electron scattering)

$$
F_{\mathrm{rad}}=K_{\mathrm{rad}} \frac{Z \pi^{2} e^{2}}{e B} \frac{\left(\omega L_{\omega}\right) \omega_{c}}{4 \pi R^{2} c} .
$$

(The numerical coefficient $K_{\mathrm{rad}}=2$ in the case of a purely radial photon field; whereas $K_{\text {rad }}=\frac{3}{4}$ near the surface of a black body.) Comparing with the gravitational force $F_{\text {grav }}=G M M_{\mathrm{NS}} / R^{2}$ on the scattering charge, and substituting eq. (34) for $L_{X}=\int L_{\omega} d \omega$, one finds

$$
\frac{F_{\text {rad }}}{F_{\text {grav }}}=\frac{\pi K_{\text {rad }} Z p F(\pi / 2)}{8(2+p)}\left(\frac{M}{m_{p}}\right)^{-1}\left(\frac{B}{B_{\text {pole }}}\right)^{-1}\left[\frac{\left(\omega L_{\omega}\right)_{\omega_{c}}}{L_{X}}\right]\left(\frac{B_{\phi}}{B_{\theta}}\right)_{\theta=\pi / 2} .
$$

The numerical coefficient in front is $O\left(10^{-1}\right)$, and for electrons one deduces

$$
\frac{F_{\text {rad }}}{F_{\text {grav }}} \sim 10^{2}\left(\frac{R_{\text {res }}}{R_{\mathrm{NS}}}\right)^{3}\left[\frac{\left(\omega L_{\omega}\right)_{\omega_{c}}}{L_{X}}\right]\left(\frac{B_{\phi}}{B_{\theta}}\right)_{\theta=\pi / 2} \gg 1 \quad \text { (electrons). }
$$

(If the twist is very small, then internal heating can easily power a large enough surface $\mathrm{X}$-ray flux to enforce the same inequality.)

By contrast, ions will interact resonantly with X-rays at the surface of the star in the presence of $\sim 10^{14}-10^{15} \mathrm{G}$ magnetic fields, and one deduces

$$
\frac{F_{\text {rad }}}{F_{\text {grav }}} \sim 0.1\left(\frac{Z}{A}\right)\left[\frac{\left(\omega L_{\omega}\right) \omega_{c}}{L_{X}}\right]\left(\frac{B_{\phi}}{B_{\theta}}\right)_{\theta=\pi / 2} \lesssim 1 \quad \text { (ions). }
$$


(Atoms of large atomic number $Z$ will become bound in molecular chains in magnetarstrength fields: Lai \& Salpeter 1997; Thompson et al. 2000.)

The radiation force on the ions can overcome gravity at the surface of the star only if the luminosity $\left(\omega L_{\omega}\right)_{\omega_{c, p}}$ of X-rays at the surface cyclotron frequency is much higher than eq. (34). One possible example of such a situation is the burst from SGR 1900+14 on 29 August 1998, which showed an extended faint tail of X-ray emission with a very hard spectrum $(k T \gtrsim 4 \mathrm{keV})$. That tail may represent transient cooling of a relatively small surface hotspot following the dissipation of a very hot magnetospheric plasma (Ibrahim et al. 2001). The radiative force at the line would also be increased in the presence of surface heating by magnetospheric charges; but the mildly relativistic translational motion of the ions would quickly shift them out of resonance with the surface cyclotron frequency. Finally, it has been suggested that in some magnetar candidates, the steep high energy spectral tail extends to frequencies well below $\hbar \omega \sim 1 \mathrm{keV}$ (Kulkarni et al. 2001). In that case, it would be possible for the radiative force on the ions to exceed gravity beyond a certain distance from the star.

\section{B.2. Draining Suspended Material through Persistent Currents}

Notice that in the case of a neutral ion-electron plasma, the radiative force on the electrons indicated by eq. (표 $)$ could exceed gravity by more than a factor $\sim(A / Z)\left(m_{p} / m_{e}\right)$, thereby allowing plasma which is blown into the magnetosphere during an X-ray flare to be supported in the magnetosphere against gravity as the X-ray flux returns to the baseline value. However, the mass of plasma which can be so supported (in e.g. a thin disk near the magnetic equator: Zheleznyakov \& Serber 1994) is small enough that it would quickly be drained by even a relatively weak magnetospheric current.

To estimate the mass which can be stored in the magnetosphere, we balance the radiation pressure normal to the 'disk' against the normal component of gravity at a scale height $h$ above the magnetic equator. The radiation pressure depends on the angular distribution of the radiation. If the magnetosphere is twisted then, at the local cyclotron resonance, a significant fraction of any central radiation field will be scattered. The radiation pressure is $\sim \frac{1}{3}$ of the energy density of the scattered radiation. At any radius in the disk, the scattered radiation will interact with the suspended particles within a frequency range $\Delta \omega / \omega \sim h / R$, which is centered at the cyclotron frequency at the disk mid-plane. We therefore have

$$
\frac{1}{3} \frac{\left(\omega L_{\omega}\right)_{\omega_{c}}}{4 \pi R^{2} c}\left(\frac{h}{R}\right) \lesssim \Sigma \frac{G M_{\mathrm{NS}}}{R^{2}}\left(\frac{h}{R}\right) .
$$


The maximum surface density which can be supported against gravity by resonant scattering is then

$$
\Sigma \lesssim \frac{\left(\omega L_{\omega}\right)_{\omega_{c}}}{12 \pi G M_{\mathrm{NS}} c} .
$$

This estimate corresponds self-consistently to a large optical depth to resonant scattering $\tau_{\text {res }} \sim F_{\text {rad }} / F_{\text {grav }}$.

The flux of charges across the magnetic equator at radius $R$ is $J \sim$ $(c / 4 \pi)\left(2 B_{\theta} / R\right)\left(B_{\phi} / B_{\theta}\right)_{\theta=\pi / 2}$. If a source of charges is available in the magnetosphere, it is energetically favorable for the current to tap it (instead of being drawn from charges lifted off the neutron star surface). Thus, one can expect the 'disk' to be drained by the current, in a very short time:

$$
\begin{aligned}
t_{\text {drain }}=\frac{2 \Sigma / m_{p}}{J / e} & \sim \frac{R_{\mathrm{NS}}}{c}\left(\frac{\left(\omega L_{\omega}\right)_{\omega_{c}}}{3 G M_{\mathrm{NS}} B_{\text {pole }} m_{p} c / e}\right)\left(\frac{R}{R_{\mathrm{NS}}}\right)^{4}\left(\frac{B_{\phi}}{B_{\theta}}\right)_{\theta=\pi / 2}^{-1} \\
& \sim 0.01\left[\frac{\left(\omega L_{\omega}\right)_{\omega_{c}}}{L_{X}}\right]\left(\frac{R}{10 R_{\mathrm{NS}}}\right)^{4} \mathrm{s.}
\end{aligned}
$$

Notice that this expression has no explicit dependence on the magnitude of the twist, if the surface X-ray flux is powered self-consistently by the impact of magnetospheric charges.

Matter can also be suspended by the centrifugal force beyond the corotation radius,

$$
R_{\mathrm{co}}=\left(G M_{\mathrm{NS}}\right)^{1 / 3} \Omega_{\mathrm{NS}}^{-2 / 3}
$$

even if the radiative force is weaker than gravity. The maximum column density which can be so suspended can be estimated by balancing the ram pressure of material corotating with the star, with the local magnetic tension (Thompson 2000; Ibrahim et al. 2001),

$$
\frac{\Sigma}{2 h}\left(R_{\mathrm{co}} \Omega_{\mathrm{NS}}\right)^{2} \simeq \frac{B_{\theta}^{2}\left(R_{\mathrm{co}}\right)}{8 \pi}
$$

Here $h$ is the scale height of the plasma above the magnetic equator. Substituting eq. (B9), and re-expressing $\Sigma$ in terms of a Thomson optical depth, we obtain

$$
\tau_{T} \simeq \frac{\Sigma \sigma_{T}}{m_{p}}=2\left(\frac{B_{\text {pole }}}{10^{15} \mathrm{G}}\right)^{2}\left(\frac{h / R_{\mathrm{co}}}{10^{-2}}\right)\left(\frac{P}{6 \mathrm{~s}}\right)^{-8 / 3}
$$

(for $M_{\mathrm{NS}}=1.4 M_{\odot}$ and $R_{\mathrm{NS}}=10 \mathrm{~km}$ ). The scale height can be estimated as

$$
\frac{h\left(R_{\mathrm{co}}\right)}{R_{\mathrm{co}}} \sim\left(\frac{k T}{m_{p} g\left(R_{\mathrm{co}}\right) R_{\mathrm{co}}}\right)^{1 / 2}
$$


where the temperature of the plasma is reduced from the surface value by the factor $T / T_{S} \sim\left(h / R_{\mathrm{co}}\right)^{1 / 4}\left(R_{\mathrm{co}} / R_{\mathrm{NS}}\right)^{-1 / 2}$. This gives

$$
\frac{h\left(R_{\mathrm{co}}\right)}{R_{\mathrm{co}}} \sim 0.004\left(\frac{k T_{S}}{0.5 \mathrm{keV}}\right)^{4 / 7}\left(\frac{P}{6 \mathrm{~s}}\right)^{4 / 21} .
$$

The normalization $B_{\text {pole }} \sim 10^{15} \mathrm{G}$ of the surface dipole field in eq. (B11) is appropriate to the actively bursting SGRs $1806-20$ and $1900+14$, if they have purely dipolar magnetic fields (Kouveliotou et al. 1998, 1999). However, we have seen (in $\S 6$ ) that the real value of $B_{\text {pole }}$ is reduced if the magnetic field decreases with radius more slowly than a dipole, $\mathrm{B} \sim R^{-(2+p)}$ with $p<1$. This also has the effect of increasing the mass which can be contained by the magnetic tension against the centrifugal force. For a fixed $P$ and $\dot{P}$, the net effect is to re-scale $B_{\text {pole }}$ from the magnetic dipole value by the factor

$$
B_{\text {pole }} \rightarrow\left(\frac{\Omega R_{\mathrm{co}}}{c}\right)^{1-p} B_{\text {pole }}(\mathrm{MDR})<B_{\text {pole }}(\mathrm{MDR})
$$

This factor is $0.55(P / 6 \mathrm{~s})^{-0.05}$ in the case $p=0.85$. Combining it with eq. (B13) in eq. (B11), we conclude that the suspended matter can maintain a modest optical depth to Thomson scattering, $\tau_{T}>1$, when $B_{\text {pole }}(\mathrm{MDR}) \gtrsim 2 \times 10^{15} \mathrm{G}$.

Finally, let us write down the time for a persistent current to drain this suspended material. It is

$$
t_{\text {drain }}=4\left(\frac{B_{\text {pole }}}{10^{15} \mathrm{G}}\right)\left(\frac{h / R_{\mathrm{co}}}{10^{-2}}\right)\left(\frac{B_{\phi}}{B_{\theta}}\right)_{\theta=\pi / 2}^{-1} \quad \mathrm{yr},
$$

assuming $M_{\mathrm{NS}}=1.4 M_{\odot}$ and $R_{\mathrm{NS}}=10 \mathrm{~km}$. Substituting once again eqs. (B13) and $(\mathrm{B} 14)$, one infers a drainage time $t_{\text {drain }} \sim 1\left(B_{\text {pole }} / 10^{15} \mathrm{G}\right)\left(B_{\phi} / B_{\theta}\right)_{\theta=\pi / 2}^{-1}$ yr (quoted here for $p=0.85)$. Of course, the density of suspended material may be high enough for it to spin outward from the star through the action of the centrifugal force, and settle into a rotationally supported disk (Thompson 2000).

\section{REFERENCES}

Arons, J. 1992, ApJ, 388, 561

Banas, K.R., Hughes, J.P., Bronfman, L., \& Nyman, L.A. 1997, ApJ, 480, 607

Basko \& Sunyaev 1975, A\&A, 42, 311

Blandford, R.D. \& Payne, D.G. 1981, MNRAS, 194, 1033 
Burrows, A. 1987, ApJ, 318, L57

Camilo, F., Kaspi, V.M., Lyne, A.G., Manchester, R.N., Bell, J.F., D'Amico, N., McKay, N.P.F., \& Crawford, F. 2000, ApJ, 541, 367

Canuto, V., Lodenquai, J., \& Ruderman, M. 1971, Phys. Rev. D3, 2303

Cline, T.L. 1982, in Gamma-Ray Transients and Related Astrophysical Phenomena, ed. R.E. Lingenfelter, H.S. Hudson \& D.M. Worrall (New York: AIP), p. 17

Deufel, B., Dullemond, C.P., \& Spruit H.C. 2001, A\&A, in press (astro-ph/0108438)

Duncan, R.C. \& Thompson, C., 1992, ApJ, 392, L9 (DT92)

Feroci, M. et al. 1999, ApJ, 515, L9

Feroci, M., Hurley, K., Duncan, R.C., \& Thompson, C., ApJ, 549, 1021

Frail, D.A. 1998, in The Many Faces of Neutron Stars, ed. R. Bucchieri, J. van Paradijs, \& M.A. Alpar (Dordrecht: Kluwer), p. 179

Frail, D.A., Kulkarni, S.R., \& Bloom, J.S. 1998, Nature, 398, 127

Fuchs Y., Mirabel, F., Chaty, S., Claret, A., Cesarsky, C.J., \& Cesarsky, D.A. 1999, A\&A, 350,891

Gaensler, B.M., Slane, P.O., Gotthelf, E.V., \& Vasisht G. 2001, ApJ, 559, 963

Gavriil, F.P. \& Kaspi, V.M. 2001, ApJ, submitted astro-ph/0107422)

Goldreich, P. \& Julian, W.H. 1969, ApJ, 157, 869

Golenetskii, S.V., Aptekar, R.L., Guryan, Y.A., Ilinskii, V.N., \& Mazets, E.P. 1987, Sov. Astron. Lett., 13, 166

Gotthelf E.V., Vasisht, G. \& Dotani, T. 1999, ApJ, 522, L49

Haberl, F., Motch, C., Buckley, D.A.H., Zickgraf, F.-J., \& Pietsch, W. 1997, A\&A, 326, 662

Hambaryan, V., Hasinger, G., Schwope, A.D., \& Schulz, N.S. 2001, A\&A, in press (astro-ph/0110365)

Harding, A.K., Contopoulos, I., \& Kazanas, D. 1999, ApJ, 525, L125

Herold, H., Ruder, H., \& Wunner, G. 1982, A\&A, 115, 90 
Heyl, J.S. \& Hernquist, L. 1997, ApJ, 489, L67

Heyl, J.S. \& Hernquist, L. 1998, MNRAS, 297, L69

Heyl, J.S. \& Kulkarni, S.R. 1998, ApJ, 506, L61

Heyl, J.S. \& Shaviv, N.J. 2000, MNRAS, 311, 555

Ho, W.C.G. \& Lai, D. 2001, MNRAS, 327, 1081

Hulleman, F., van Kerkwijk, M.H., \& Kulkarni, S.R. 2000, Nature, 408, 689

Hulleman, F., Tennant, A.F., van Kerkwijk, M.H., Kulkarni, S.R., Kouveliotou, C., \& Patel, S.K. 2001, ApJ, in press (astro-ph/0110172)

Hurley, K. 2000, in Proceedings of the Fifth Compton Symposium, ed. M.L. McConnell \& J.M. Ryan (New York: AIP), p. 515

Hurley, K., Cline, T., Mazets, E., Barthelmy, S., Butterworth, P., Marshall, F., Palmer, D., Aptekar, R., Golenetskii, S., Il'Inskii, V., Frederiks, D., McTiernan, J., Gold, R., \& Trombka, J. 1999a, Nature, 397, 41

Hurley, K., Kouveliotou, C., Woods, P., Cline, T., Butterworth, P., Mazets, E., Golenetskii, S., \& Frederics, D., 1999b, ApJ, 510, L107

Hurley, K., Li, P., Kouveliotou, C., Murakami, T., Ando, M., Strohmayer, T., van Paradijs, J., Vrba, F., Luginbuhl, C., Yoshida, A., \& Smith, I. 1999c, ApJ, 510, L111

Hurley, K., Kouveliotou, C., Cline, T., Mazets, E., Golenetskii, S., Frederiks, D.D., \& van Paradijs, J. 1999d, ApJ, 523, L37

Ibrahim, A.I., Strohmayer, T.E., Woods, P.M., Kouveliotou, C., Thompson, C., Duncan, R.C., Dieters, S., van Paradijs, J., \& Finger, M. 2001, ApJ, in press (astro-ph/0007043)

Iwasawa, K., Koyama, K., \& Halpern, J.P. 1992, PASJ, 44, 9

Kaplan, D.L., Kulkarni, S.R., van Kerkwijk, M.H., Rothschild, R.E., Lingenfelter, R.L., Marsden, D., Danner, R., \& Murakami, T. 2001a, ApJ, 556, 399

Kaplan, D.L., Kulkarni, S.R., Frail D.A., \& van Kerkwijk, M.H. 2001b, ApJ, submitted (astro-ph/0107519)

Kaplan, D.L., Fox, D.W., Kulkarni, S.R., Gotthelf, E.V., Vasisht, G., \& Frail D.A. 2001c, ApJ, submitted (astro-ph/0108195) 
Kaspi, V.M., Chakrabarty, D., \& Steinberger, J. 1999, ApJ525, L33

Kaspi, V.M., Gavriil, F.P., Chakrabarty, D., Lackey, J.R., \& Muno, M.P. ApJ, 558, 253

Keil, W., Janka, H.-T., \& Mueller, E. 1996, ApJ, 473, L111

Kouveliotou, C., et al. 1998, Nature, 393, 235

Kouveliotou, C., et al. 1999, ApJ, 510, L115

Kulkarni, S.R. \& Frail, D.A. 1993, Nature, 365, 33

Kulkarni, S.R., Frail, D.A., Kassim, N.E., Murakami, T., \& Vasisht, G. 1994, Nature, 368, 129

Kulkarni, S.R. \& van Kerkwijk, M.H. 1998, A\&A, 507, L49

Kulkarni, S.R., Kaplan, D.L., Marshall, H.L., Frail, D.A., Murakami, T., \& Yonetoku, D. 2001, preprint

Lai, D. \& Ho, W.C.G. 2001, preprint (astro-ph/0108127)

Lai, D. \& Salpeter, E.E. 1997, ApJ, 491, 270

Lynden-Bell, D. \& Boily, C. 1994, MNRAS, 267, 146

Lyutikov, M. \& Thompson C. 2001, in progress

Marsden, D., Rothschild, R.E., \& Lingenfelter, R. 1999, ApJ, 520, L107

Marsden, D. \& White, N.E. 2001, ApJ, 551, L155

Mazets, E.P., et al., 1979, Nature, 282, 587

Mazets, E.P., et al., 1999, preprint astro-ph/9905196 v2)

Mereghetti, S. 2000, in 'The Neutron Star - Black Hole Connection', ed. V. Connaughton, C. Kouveliotou, J. van Paradijs, \& J. Ventura (Dordrecht: Reidel), in press (astro-ph/9911252)

Michel, F.C. 1991, 'Theory of Neutron Star Magnetospheres', (U. Chicago Press)

Mestel, L. 1993, in Pulsars as Physics Laboratories, ed. R.D. Blandford, A. Hewish, A.G. Lyne, \& L. Mestel (Oxford), p. 93

Mestel, L. \& Pryce, M.H.L. 1992, MNRAS, 254, 355 
Mitrofanov, I.G. \& Pavlov, G.G. 1982, MNRAS, 200, 1033

Murakami, T., Tanaka, Y., Kulkarni, S. R., Ogasaka, Y., Sonobe, T., Ogawara, Y., Aoki, T., \& Yoshida, A. 1994, Nature, 368, 127

Murakami, T. et al. 1999, ApJ, 510, L122

Nelson, R.W., Salpeter, E.E., \& Wasserman, I. 1993, ApJ, 418, 874

Nelson, R.W., Wang, J.C.L., Salpeter, E.E., \& Wasserman, I. 1995, ApJ, 438, L99

Neuhaüser, R. \& Trümper, J.E. 1999, A\&A, 343, 151

Oosterbroek, T., Parmar, A.N., Mereghetti, S., \& Israel, G.L. 1998, A\&A, 334, 925

Özel, F. 2001, ApJ, submitted (astro-ph/0103227)

Pacini, F. 1967, Nature, 216, 567

Paczyǹski, B., 1992, Acta Astron., 42, 145

Palmer, D.M. 2001, in 'Soft Gamma Repeaters: The Rome 2001 Mini-Workshop', ed. M. Feroci, S. Mereghetti, \& L. Stella, in press (astro-ph/0103404)

Paul, B., Kawasaki, M., Dotani, T., \& Nagase, F. 2000, ApJ, 537, 319

Pivovaroff, M.J., Kaspi, V.M., \& Camilo, F. 2000, ApJ, 535, 379

Psaltis, D., Özel, F., \& DeDeo, S. 2000, ApJ, 544, 390

Rajagopal, M. \& Romani, R.W. 1997, ApJ, 491, 296

Reisenegger, A. \& Goldreich, P. 1992, ApJ, 395, 250

Ruderman, M.A. \& Sutherland, P.G. 1975, ApJ, 195, 51

Schaeffer, R., Reeves, H., \& Orland, H. 1982, ApJ, 254, 688

Scharlemann, E.T., Arons, J., \& Fawley, W.M. 1978, ApJ, 222, 297

Sincell, M.W. \& Krolik, J.H. 1992, ApJ, 395, 553

Thompson, C. 2000, in The Neutron Star-Black Hole Connection, ed. V. Connaughton, C. Kouveliotou, J. van Paradijs, \& J. Ventura (Dordrecht: Reidel), in press (astro-ph/0010016) 
Thompson, C. 2001, in Soft Gamma Repeaters: The Rome 2000 Mini-Workshop, ed. M. Feroci, S. Mereghetti, \& L. Stella, in press (astro-ph/0110679)

Thompson, C. \& Blaes, O. 1998, Phys. Rev. D, 57, 3219

Thompson, C. \& Duncan, R.C. 1993, ApJ, 408, 194 (TD93)

Thompson, C. \& Duncan, R.C., 1995, MNRAS, 275, 255 (TD95)

Thompson, C. \& Duncan, R.C. 1996, ApJ, 473, 322 (TD96)

Thompson, C., Duncan, R.C., Woods, P.M., Kouveliotou, C., Finger, M.H., \& van Paradijs, J. 2000, ApJ, 543, 340

Thompson, C. \& Duncan, R.C. 2001, ApJ, 561, 000 (astro-ph/0110675)

Thompson, C. \& Murray, N.W. 2001, ApJ, 560, 000 (astro-ph/0105425)

Treves, A., Popov, S.B., Colpi, M., Prokhorov, M.E., \& Turolla R. 2000, in X-ray astronomy 2000, ed. R. Giacconi, L. Stella, \& S. Serio (San Francisco: ASP), in press

van Kerkwijk, M.H., Kulkarni, S.R., Matthews, K., \& Neugebauer, G. 1995, ApJ, 444, L33

Vrba, F.J., Henden, A.A., Luginbuhl, C.B., Guetter, H.H., Hartmann, D.H., \& Klose, S. 2000, ApJ, 533, L17

Wolfson, R. 1995, ApJ, 443, 810

Woltjer, L. 1958, ApJ, 128, 384

Woods, P., Kouveliotou, C., van Paradijs, J., Finger, M.H. \& Thompson, C. 1999a, ApJ, 518, L103

Woods, P., Kouveliotou, C., van Paradijs, J., Hurley, K., Kippen, R., Finger, M.H., Briggs, M., Dieters, S., \& Fishman, G. 1999b, ApJ, 519, L139

Woods, P.M., Kouveliotou, C., van Paradijs, J., Finger, M.H., Thompson, C., Duncan, R.C., Hurley, K., Strohmayer, T., Swank, J., \& Murakami, T. 1999c, ApJ, 524, L55

Woods, P.M., Kouveliotou, C., Finger, M.H., Gögüs, E., Scott, D.M., Dieters, S., Thompson, C., Duncan, R. C., Hurley, K., Strohmayer, T., Swank, J., \& Murakami, T. 2000, ApJ, 535, L55

Woods, P.M., Kouveliotou, C., Gogus, E., Finger, M.H., Swank, J., Smith, D.A., Hurley, K., \& Thompson, C. 2001a, ApJ, 552, 748 
Woods, P.M., Kouveliotou, C., Gougus, E., Finger, M.H., Swank, J., Markwardt, C.B., Hurley, K., \& van der Klis, M. 2001b, ApJ, submitted (astro-ph/0109361)

Zane, S., Turolla, R., \& Treves, A. 2000, ApJ, 537, 387

Zane, S., Turolla, R., Stella, L., \& Treves, A. 2001, ApJ, 560, 384

Zel'dovich, Y.B. \& Shakura, N.I. 1969, Soviet Astron.-AJ, 13, 175

Zheleznyakov, V.V. \& Serber, A.V. 1994, Space Sci. Rev., 68, 


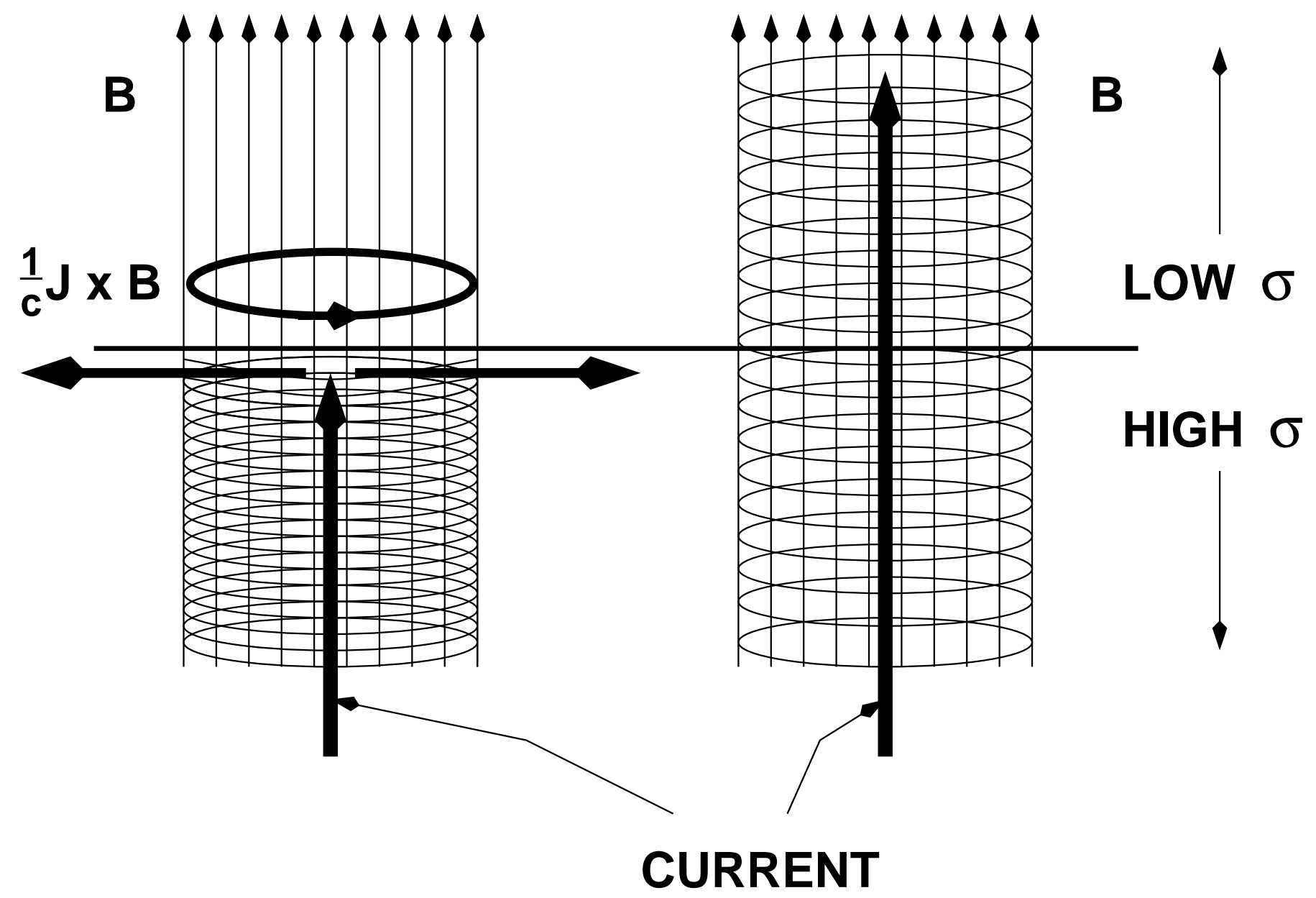


Fig. 1.- A twisted magnetic field is anchored in the highly conducting interior of a degenerate star. The twist is initially confined to the interior of the star, so that the current closes at the surface by flowing across the magnetic field. The resulting $\frac{1}{c} \mathbf{J} \times \mathbf{B}$ force causes the liquid near the surface to rotate, so as to distribute the twist more uniformly along the magnetic field lines. The net effect is to force the current to flow out of the star, into its 'magnetosphere'. In the case of a magnetar, this process may be partly stabilized by the rigidity of the crust, so that the external field twists up intermittently (giving rise to SGR flares). 


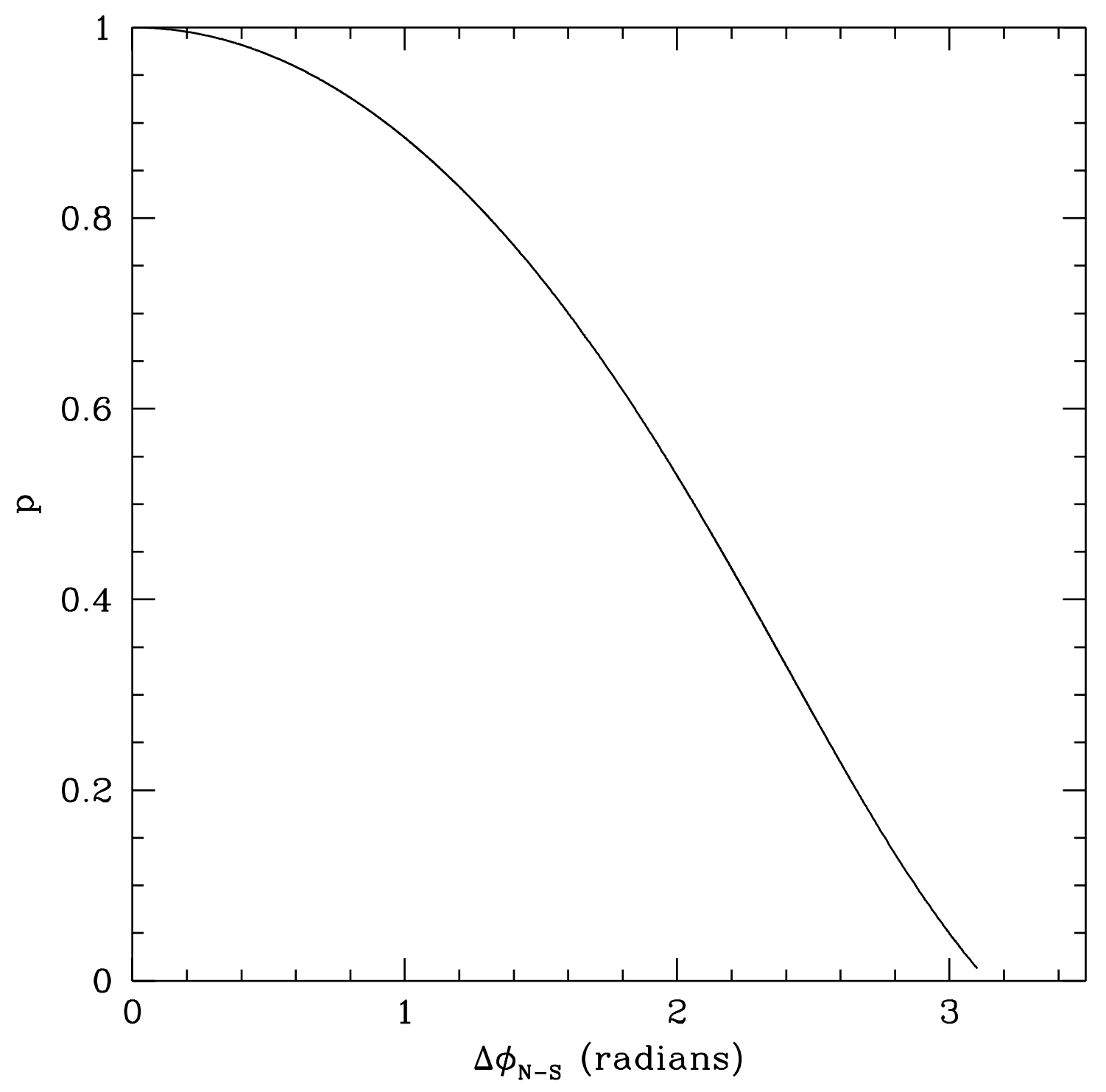

Fig. 2.- The radial index $p$ of the magnetic flux function $\mathcal{P}$ (eq. [3]) is plotted versus the net twist angle $\Delta \phi_{\mathrm{N}-\mathrm{S}}=\Delta \phi(\theta \rightarrow 0)$ between the north and south magnetic poles (eq. [B]). 

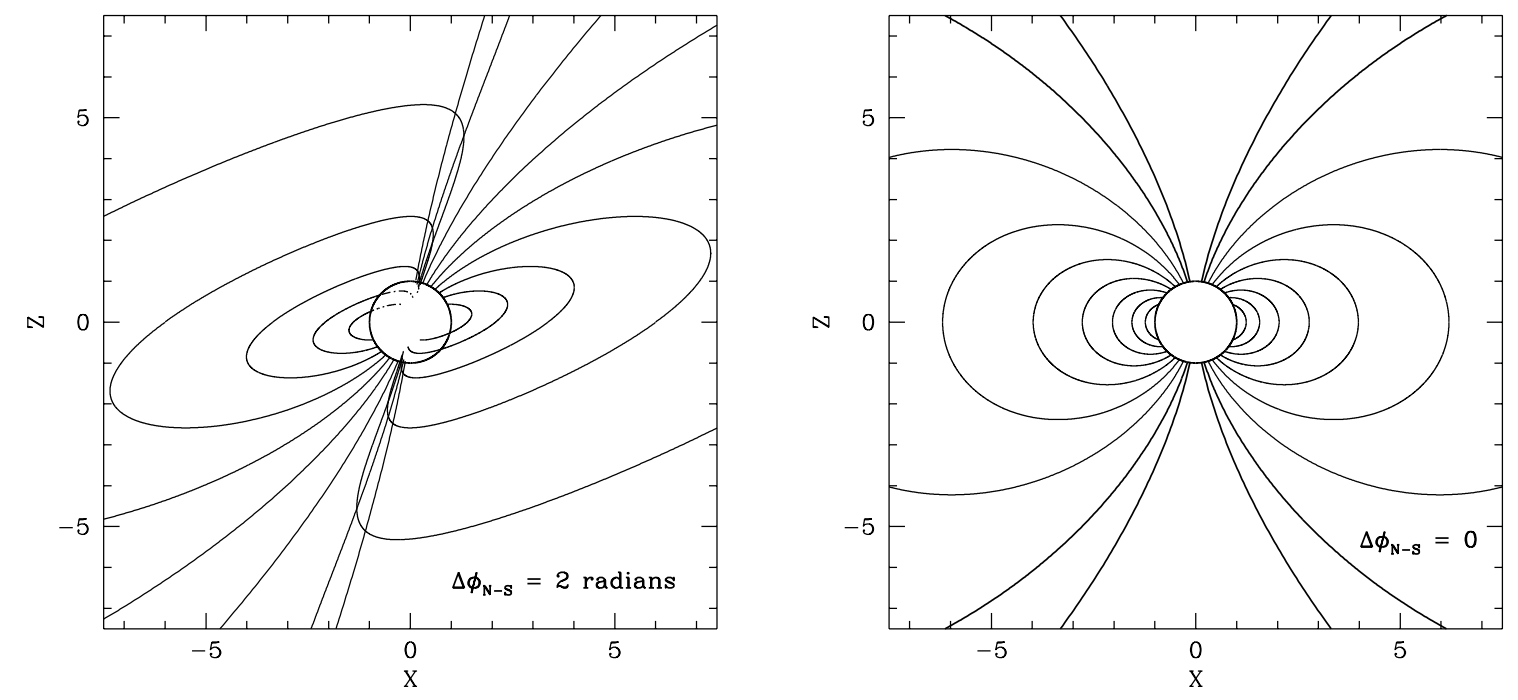

Fig. 3.- An example of a twisted, self-similar, force-free magnetosphere, with net twist angle $\Delta \phi_{\mathrm{N}-\mathrm{S}}=2$ radians. Only a small number of field lines are plotted here. The field lines protruding from the top right and bottom left corners of the star are anchored in the $X-Z$ plane, at regular intervals $\Delta \mu=0.1$. Dashed lines indicate that the field is projected behind the star. A pure dipole is shown for comparison. 


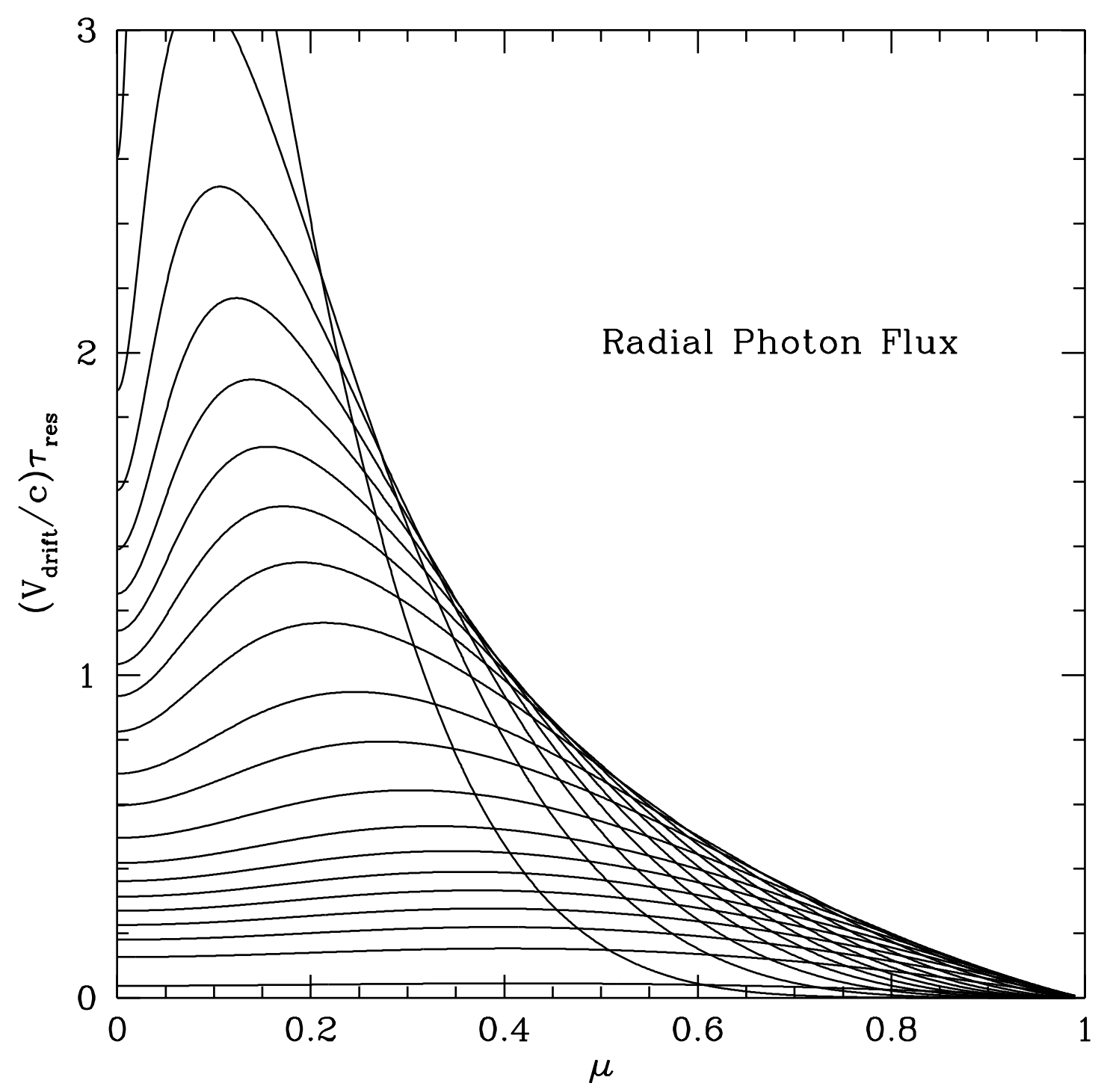

Fig. 4.- Optical depth $\tau_{\text {res }}$ to scattering at the cyclotron resonance (eq. 24]) experienced by radially streaming photons, as a function of magnetic co-latitude $\mu=\cos \theta$ and the drift speed $V_{\text {drift }}$. This optical depth is calculated in the approximation that the charges are static. The various curves correspond to different twist angles $\Delta \phi_{\mathrm{N}-\mathrm{S}}$; the peak value of $\tau_{\text {res }}\left(V_{\text {drift }} / c\right)$ is $\sim 0.6$ when the twist is $\Delta \phi_{\mathrm{N}-\mathrm{S}}=1.5$ radians. Within a self-similar twisted magnetosphere, this optical depth is independent of the radius, frequency, or the charge/mass ratio of the scattering particle (as long as the cyclotron resonance sits outside the spherical surface in which the magnetic field is anchored). If the current is supported by more than one species of charge carrier, as is generally the case, the plotted value of $\tau_{\text {res }}$ must be multiplied by the fraction of the current carried by the species of scattering charge. 


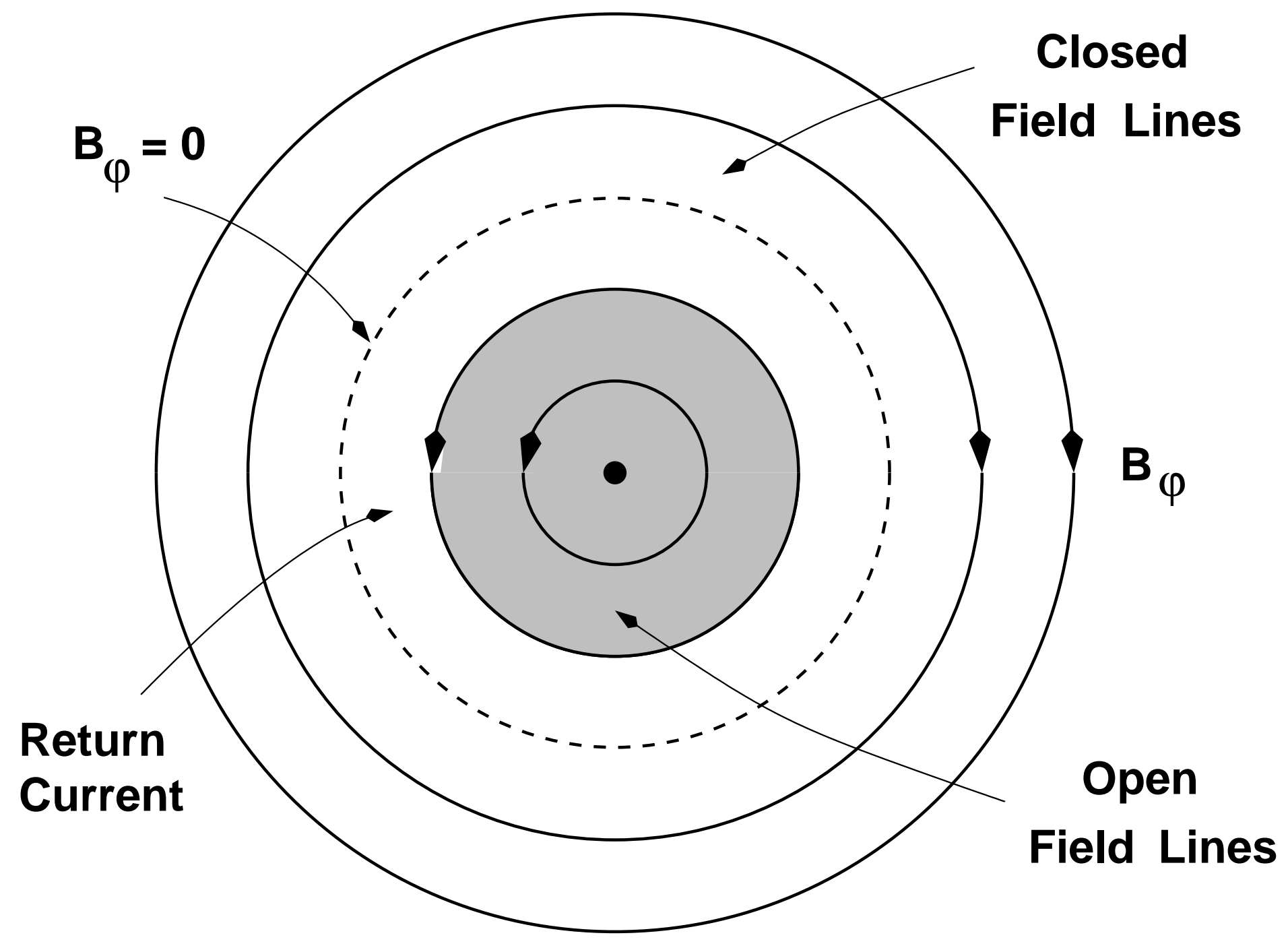


Fig. 5.- In one magnetic hemisphere, a space-charge limited flow will generate a current of the opposite sign to the current flowing on the closed, twisted field lines. The toroidal component of the field will then have an opposing sense on the open and closed field lines. In the absence of pairs, this may cause a cancellation of the twist in the intermediate regions of the magnetosphere. An electron-positron pair cascade allows the density of currentcarrying particles to build up, so that the Goldreich-Julian charge density $-\boldsymbol{\Omega} \cdot \mathbf{B} / 2 \pi c$ can be maintained even as the current flows backward compared with a pure space-charge limited flow. 


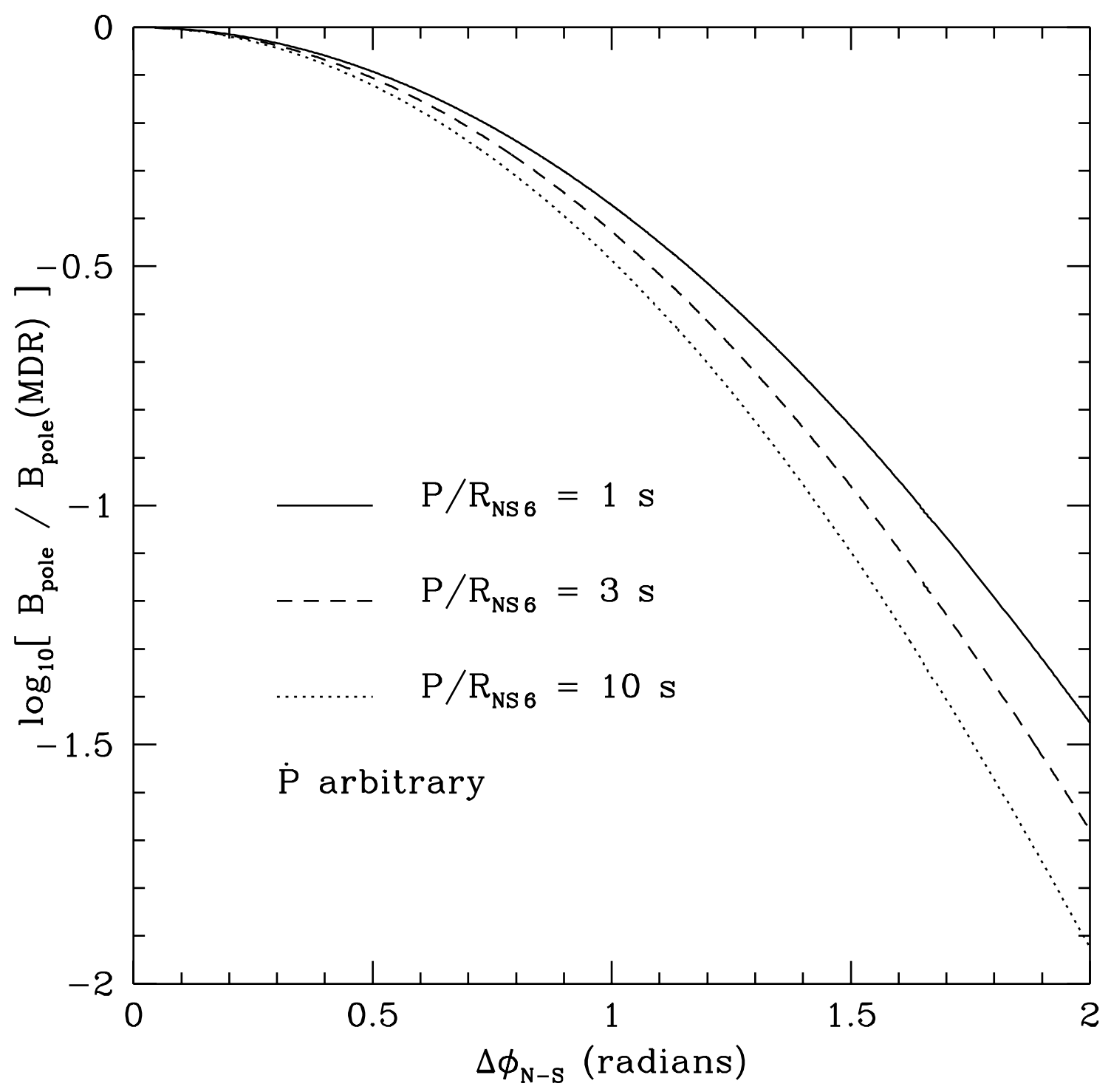

Fig. 6.- Polar surface magnetic field, as inferred from a measured spin period $P$ and period derivative $\dot{P}$, compared with the classical magnetic dipole formula (eq. 450) evaluated at $\sin \alpha=1$. 